Publ. RIMS, Kyoto Univ.

42 (2006), 221-322

\title{
Semi-graphs of Anabelioids
}

\author{
By
}

Shinichi MochIzUKI*

\begin{abstract}
In this paper, we discuss various "general nonsense" aspects of the geometry of semi-graphs of profinite groups [cf. [Mzk3], Appendix], by applying the language of anabelioids introduced in [Mzk4]. After proving certain basic properties concerning various commensurators associated to a semi-graph of anabelioids, we show that the geometry of a semi-graph of anabelioids may be recovered from the category-theoretic structure of certain naturally associated categories - e.g., "temperoids" [in essence, the analogue of a Galois category for the "tempered fundamental groups" of [André]] and "categories of localizations". Finally, we apply these techniques to obtain certain results in the absolute anabelian geometry [cf. [Mzk3], [Mzk8]] of tempered fundamental groups associated to hyperbolic curves over $p$-adic local fields.
\end{abstract}

\section{Contents}

§0. Notations and Conventions

$\S 1$. Zariski's Main Theorem for Semi-graphs

$\S 2$. Commensurability Properties

$\S 3 . \quad$ The Tempered Fundamental Group

$\S 4$. Categories of Localizations

$\S 5$. Arithmetic Semi-graphs of Anabelioids

$\S 6 . \quad$ Tempered Anabelian Geometry

Appendix : Quasi-temperoids

Communicated by T. Kawai. Received October 6, 2004. Revised April 18, 2005, October $11,2005$.

2000 Mathematics Subject Classification(s): 14H30, 14H25.

* Research Institute for Mathematical Sciences, Kyoto University, Kyoto 606-8502, Japan. e-mail: motizuki@kurims.kyoto-u.ac.jp 
Index

References

\section{Introduction}

In this paper, we continue to pursue the theme of categorical representation of scheme-theoretic geometries, which played a central role in [Mzk6], [Mzk7], as well as in the previous anabelian work of the author [e.g., [Mzk2], [Mzk3], [Mzk5], [Mzk8]]. The original motivation of the present work lies in the problem of finding an appropriate and efficient way of representing, via categories, the geometry of "formal localizations" of hyperbolic curves over p-adic local fields. Here, we use the term "formal localizations" to refer to the localizations of the $p$-adic formal completion of a stable log curve over the ring of integers of a $p$-adic local field obtained by completing along the irreducible components and nodes of the geometric logarithmic special fiber specified by some sub-semi-graph of the "dual semi-graph with compact structure" [cf. [Mzk3], Appendix] associated to this geometric logarithmic special fiber. Since the geometry of such formal localizations is substantially reflected in the geometry of localizations of the semi-graph of profinite groups [cf. [Mzk3], Appendix] associated to this geometric logarithmic special fiber, it is thus natural, from the point of view of the goal of categorical representation of this geometry of formal localizations, to study the geometry of this semi-graph of profinite groups. Moreover, when working with profinite groups as "geometric objects", it is natural to apply the language of anabelioids introduced in [Mzk4].

The main results of this paper may be summarized as follows:

(1) In $\S 1$, we study the geometry of semi-graphs, and in particular, expose a proof related to the author by M. Matsumoto of a sort of analogue for certain types of morphisms of finite semi-graphs of "Zariski's main theorem" in scheme theory [cf. Theorem 1.2]. This result has some interesting group-theoretic consequences related to the author by A. Tamagawa [cf. Corollary 1.6]; in addition, it admits an interesting interpretation from a more "arithmetic" point of view [cf. Remark 1.5.1].

(2) In $\S 2$, we begin our study of the geometry of semi-graphs of anabeloids. Our main result [cf. Corollary 2.7] concerns certain properties of the commensurator in the profinite fundamental group associated to a graph of 
anabelioids of the various subgroups associated to subgraphs of the given graph of anabelioids.

(3) In $\S 3$, we take up the study of "tempered fundamental groups" [i.e., roughly speaking, fundamental groups that correspond to coverings dominated by the composite of an arbitrary finite covering and a [not necessarily finite] covering of "some" associated semi-graph — cf. [André]], by working with "temperoids", i.e., the analogue for tempered fundamental groups of Galois categories [in the case of profinite groups]. Our main result [cf. Theorem 3.7; Corollary 3.9] states that for certain kinds of graphs of anabelioids, the vertices (respectively, edges) of the underlying graph may be recovered from the associated tempered fundamental group as the [conjugacy classes of] maximal compact subgroups (respectively, nontrivial intersections of distinct maximal compact subgroups) of this tempered fundamental group. We then apply this result to show, in the case of hyperbolic curves over p-adic local fields, that the entire dual semi-graph with compact structure may be recovered solely from the geometric tempered fundamental group of such a curve [cf. Corollary 3.11].

(4) Although the tempered fundamental group furnishes perhaps the most efficient way of reconstructing a graph of anabelioids from a naturally associated category, in $\S 4$, we examine another natural approach to this problem, via categories of localizations. This approach is motivated partly by the geometry of formal localizations of stable log curves referred to above, and partly by the naive observation that given a semi-graph of anabelioids, it is natural to "localize" not just by considering coverings, but also by "physically localizing on the underlying semi-graph". After studying various basic properties of such categories of localizations [including some interesting properties that follow from "Zariski's main theorem for semi-graphs" cf. Proposition 4.4, (i), (ii)], we show that, given a graph of anabelioids satisfying certain properties, the original graph of anabelioids may be recovered functorially from its associated category of localizations [cf. Theorem 4.8]. One recurrent theme in the theory of $\S 4$ [which is consistent with the more general theme of "categorical representation of scheme-theoretic geometries" referred to above] is the idea that the geometry that is hidden in such a category of localizations may be developed, using entirely category-theoretic notions, in a fashion that is remarkably reminiscent of classical scheme theory - cf. the application of "Zariski's main theorem for semi-graphs" in Proposition 4.4, (i), (ii); the "valuative criterion" of Proposition 4.6. 
(5) In $\S 3,4$, we considered semi-graphs of anabelioids that are not equipped with "Galois actions". Thus, in $\S 5$, we generalize the [more efficient] theory of $\S 3$ [instead of the theory of $\S 4$, since this becomes somewhat cumbersome] to the "arithmetic" situation that arises in the case of a hyperbolic curve over a $p$-adic local field, i.e., of a semi-graph of anabelioids equipped with an "arithmetic action" by a profinite group. The translation of the theory of $\S 3$ into its "arithmetic analogue" in $\S 5$ is essentially routine, once one replaces, for instance, "maximal compact subgroups" by "arithmetically maximal compact subgroups" [cf. Theorem 5.4].

(6) In $\S 6$, we consider the tempered analogue of the absolute anabelian geometry developed in [Mzk8]. In particular, we show that in many respects, this tempered analogue is essentially equivalent to the original profinite version [cf. Theorem 6.6], and, moreover, that the various absolute anabelian results of [Mzk8] concerning decomposition groups of closed points - in particular, a sort of "weak section conjecture" — also hold in the tempered case [cf. Theorem 6.8; Corollaries 6.9, 6.11]. This is particularly interesting in that the tempered version exhibits, in a very explicit way, the geometry of this "weak section conjecture" in a fashion that is quite reminiscent of the "discrete real section conjecture" of [Mzk5], §3.2 [cf. Remark 6.9.1], i.e., relative to the well-known analogy between geodesics on trees [cf., e.g., Lemma 1.8, (ii); [Serre]] and geodesics in Riemannian "straight line spaces" [i.e., Riemannian spaces satisfying the condition $\left(^{*}\right)$ of [Mzk5], §3.2].

(7) In the Appendix, we discuss a slight generalization of the notions of "temperoids" and "anabelioids" that sometimes appears in practice, especially when one wishes to consider, from the point of view of the categories discussed in the present paper, the "stack-theoretic analogue" of various "scheme-theoretic notions" [cf., e.g., Remarks 4.1.2, 4.8.4]. The main result of the Appendix [cf. Theorem A.4] states that a temperoid may be reconstructed category-theoretically from a certain type of subcategory of the temperoid [i.e., a "quasi-temperoid"]. This sort of technical result is also of interest, relative to the analogy between temperoids and anabelioids, in the context of the theory of cores of anabelioids developed in [Mzk4].

Finally, we remark that to a certain extent, this paper was conceived by the author as a piece of mathematical infrastructure, i.e., to develop the basic properties and "general nonsense" of the very "primitive" [by comparison to many modern mathematical notions] notion of a semi-graph of anabelioids in maximal possible generality. Thus, although, for instance, the exposition of $\S 2$, 
$\S 3$ could be substantially simplified if one restricts oneself to the sort of semigraphs of anabelioids that arise from stable log curves, it seemed more natural to the author to develop this theory under minimal possible hypotheses. As a result of this choice on the part of the author, the present paper contains a very large number of new terms, which may be ignored to a substantial extent on a first reading of the present paper, by assuming, for instance, that all semigraphs of anabelioids are of the sort that arise from stable log curves. Also, it is hoped that the Index provided at the end of the paper may aid in the tracking down of unknown terminology.

\section{$\S 0 . \quad$ Notations and Conventions}

\section{Topological groups}

Let $G$ be a Hausdorff topological group, and $H \subseteq G$ a closed subgroup. Let us write

$$
Z_{G}(H) \stackrel{\text { def }}{=}\{g \in G \mid g \cdot h=h \cdot g, \forall h \in H\}
$$

for the centralizer of $H$ in $G$;

$$
N_{G}(H) \stackrel{\text { def }}{=}\left\{g \in G \mid g \cdot H \cdot g^{-1}=H\right\}
$$

for the normalizer of $H$ in $G$; and

$$
C_{G}(H) \stackrel{\text { def }}{=}\left\{g \in G \mid\left(g \cdot H \cdot g^{-1}\right) \bigcap H \text { has finite index in } H, g \cdot H \cdot g^{-1}\right\}
$$

for the commensurator of $H$ in $G$. Note that: (i) $Z_{G}(H), N_{G}(H)$ and $C_{G}(H)$ are subgroups of $G$; (ii) we have inclusions

$$
H, Z_{G}(H) \subseteq N_{G}(H) \subseteq C_{G}(H)
$$

and (iii) $H$ is normal in $N_{G}(H)$.

Note that $Z_{G}(H), N_{G}(H)$ are always closed in $G$, while $C_{G}(H)$ is not necessarily closed in $G$. If $H=C_{G}(H)$, then we shall say that $H$ is commensurably terminal in $G$.

If $G$ is center-free, then we have a natural exact sequence

$$
1 \rightarrow G \rightarrow \operatorname{Aut}(G) \rightarrow \operatorname{Out}(G) \rightarrow 1
$$

[where $\operatorname{Aut}(G)$ denotes the group of automorphisms of the topological group $G$; the injective [since $G$ is center-free!] homomorphism $G \rightarrow \operatorname{Aut}(G)$ is obtained by letting $G$ act on $G$ by inner automorphisms; $\operatorname{Out}(G)$ is defined so as to 
render the sequence exact]. If $J \rightarrow \operatorname{Out}(G)$ is a homomorphism of groups, then we shall write

$$
G \stackrel{\text { out }}{\rtimes} \stackrel{\text { def }}{=} \operatorname{Aut}(G) \times \operatorname{Out}(G) J
$$

for the "outer semi-direct product of $J$ with $G$ ". Thus, we have a natural exact sequence: $1 \rightarrow G \rightarrow G \stackrel{\text { out }}{\rtimes} J \rightarrow J \rightarrow 1$.

\section{Categories}

Let $\mathcal{C}$ be a category. We shall denote the collection of objects of $\mathcal{C}$ by:

$$
\mathrm{Ob}(\mathcal{C})
$$

If $A \in \mathrm{Ob}(\mathcal{C})$ is an object of $\mathcal{C}$, then we shall denote by

$$
\mathcal{C}_{A}
$$

the category whose objects are morphisms $B \rightarrow A$ of $\mathcal{C}$ and whose morphisms (from an object $B_{1} \rightarrow A$ to an object $B_{2} \rightarrow A$ ) are $A$-morphisms $B_{1} \rightarrow B_{2}$ in $\mathcal{C}$. Thus, we have a natural functor

$$
\left(j_{A}\right) !: \mathcal{C}_{A} \rightarrow \mathcal{C}
$$

(given by forgetting the structure morphism to $A$ ). Similarly, if $f: A \rightarrow B$ is a morphism in $\mathcal{C}$, then $f$ defines a natural functor

$$
f_{!}: \mathcal{C}_{A} \rightarrow \mathcal{C}_{B}
$$

by mapping an arrow (i.e., an object of $\left.\mathcal{C}_{A}\right) C \rightarrow A$ to the object of $\mathcal{C}_{B}$ given by the composite $C \rightarrow A \rightarrow B$ with $f$. Also, we shall denote by

$$
\mathcal{C}[A] \subseteq \mathcal{C}
$$

the full subcategory determined by the objects of $\mathcal{C}$ that admit a morphism to A.

If the category $\mathcal{C}$ admits finite products, then $\left(j_{A}\right)$ ! is left adjoint to the natural functor

$$
j_{A}^{*}: \mathcal{C} \rightarrow \mathcal{C}_{A}
$$

given by taking the product with $A$, and $f_{!}$is left adjoint to the natural functor

$$
f^{*}: \mathcal{C}_{B} \rightarrow \mathcal{C}_{A}
$$


given by taking the fibered product over $B$ with $A$. We shall call an object $A \in \mathrm{Ob}(\mathcal{C})$ terminal if for every object $B \in \mathrm{Ob}(\mathcal{C})$, there exists a unique arrow $B \rightarrow A$ in $\mathcal{C}$.

We shall refer to a natural transformation between functors all of whose component morphisms are isomorphisms as an isomorphism between the functors in question. A functor $\phi: \mathcal{C}_{1} \rightarrow \mathcal{C}_{2}$ between categories $\mathcal{C}_{1}, \mathcal{C}_{2}$ will be called rigid if $\phi$ has no nontrivial automorphisms. A category $\mathcal{C}$ will be called slim if the natural functor $\mathcal{C}_{A} \rightarrow \mathcal{C}$ is rigid, for every $A \in \operatorname{Ob}(\mathcal{C})$.

If $G$ is a profinite group, then we shall denote by

$$
\mathcal{B}(G)
$$

the category of finite sets with continuous $G$-action. Thus, $\mathcal{B}(G)$ is a Galois category, or, in the terminology of [Mzk4], a connected anabelioid. Moreover, $\mathcal{B}(G)$ is slim if and only if, for every open subgroup $H \subseteq G$, we have $Z_{G}(H)=$ $\{1\}$ [cf. [Mzk4], Corollary 1.1.6, Definition 1.2.4].

A diagram of functors between categories will be called 1-commutative if the various composite functors in question are isomorphic. When such a diagram "commutes in the literal sense" we shall say that it 0 -commutes. Note that when a diagram in which the various composite functors are all rigid "1-commutes", it follows from the rigidity hypothesis that any isomorphism between the composite functors in question is necessarily unique. Thus, to state that such a diagram 1-commutes does not result in any "loss of information" by comparison to the datum of a specific isomorphism between the various composites in question.

Given two functors $\Phi_{i}: \mathcal{C}_{i} \rightarrow \mathcal{D}_{i}$ (where $i=1,2$ ) between categories $\mathcal{C}_{i}$, $\mathcal{D}_{i}$, we shall refer to a 1 -commutative diagram

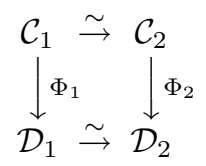

- where the horizontal arrows are equivalences of categories - as an abstract equivalence from $\Phi_{1}$ to $\Phi_{2}$. If there exists an abstract equivalence from $\Phi_{1}$ to $\Phi_{2}$, then we shall say that $\Phi_{1}, \Phi_{2}$ are abstractly equivalent.

We shall say that a nonempty [i.e., non-initial] object $A \in \mathrm{Ob}(\mathcal{C})$ is connected if it is not isomorphic to the coproduct of two nonempty objects of $\mathcal{C}$. We shall say that an object $A \in \mathrm{Ob}(\mathcal{C})$ is mobile if there exists an object $B \in \mathrm{Ob}(\mathcal{C})$ such that the set $\operatorname{Hom}_{\mathcal{C}}(A, B)$ has cardinality $\geq 2$ [i.e., the diagonal from this set to the product of this set with itself is not bijective]. We shall 
say that an object $A \in \operatorname{Ob}(\mathcal{C})$ is quasi-connected if it is either immobile [i.e., not mobile] or connected. Thus, connected objects are always quasi-connected. If every object of a category $\mathcal{C}$ is quasi-connected, then we shall say that $\mathcal{C}$ is a category of quasi-connected objects. We shall say that a category $\mathcal{C}$ is totally (respectively, almost totally) epimorphic if every morphism in $\mathcal{C}$ whose domain is arbitrary (respectively, nonempty) and whose codomain is quasi-connected is an epimorphism.

We shall say that $\mathcal{C}$ is of finitely (respectively, countably) connected type if it is closed under formation of finite (respectively, countable) coproducts; every object of $\mathcal{C}$ is a coproduct of a finite (respectively, countable) collection of connected objects; and, moreover, all finite (respectively, countable) coproducts $\amalg A_{i}$ in the category satisfy the condition that the natural map

$$
\coprod \operatorname{Hom}_{\mathcal{C}}\left(B, A_{i}\right) \rightarrow \operatorname{Hom}_{\mathcal{C}}\left(B, \coprod A_{i}\right)
$$

is bijective, for all connected $B \in \mathrm{Ob}(\mathcal{C})$. If $\mathcal{C}$ is of finitely or countably connected type, then every nonempty object of $\mathcal{C}$ is mobile; in particular, a nonempty object of $\mathcal{C}$ is connected if and only if it is quasi-connected.

If a mobile object $A \in \mathrm{Ob}(\mathcal{C})$ satisfies the condition that every morphism in $\mathcal{C}$ whose domain is nonempty and whose codomain is equal to $A$ is an epimorphism, then $A$ is connected. [Indeed, $C_{1} \amalg C_{2} \stackrel{\sim}{\rightarrow} A$, where $C_{1}, C_{2}$ are nonempty, implies that the composite map

$$
\begin{aligned}
\operatorname{Hom}_{\mathcal{C}}(A, B) \hookrightarrow \operatorname{Hom}_{\mathcal{C}}(A, B) \times \operatorname{Hom}_{\mathcal{C}}(A, B) \hookrightarrow \operatorname{Hom}_{\mathcal{C}}\left(C_{1}, B\right) \times \operatorname{Hom}_{\mathcal{C}}\left(C_{2}, B\right) \\
=\operatorname{Hom}_{\mathcal{C}}\left(C_{1} \coprod C_{2}, B\right) \stackrel{\sim}{\rightarrow} \operatorname{Hom}_{\mathcal{C}}(A, B)
\end{aligned}
$$

is bijective, for all $B \in \mathrm{Ob}(\mathcal{C})$.]

If $\mathcal{C}$ is a category of finitely or countably connected type, then we shall write

$$
\mathcal{C}^{0} \subseteq \mathcal{C}
$$

for the full subcategory of connected objects. [Note, however, that in general, objects of $\mathcal{C}^{0}$ are not necessarily connected - or even quasi-connected - as objects of $\mathcal{C}^{0}$ !] On the other hand, if, in addition, $\mathcal{C}$ is almost totally epimorphic, then $\mathcal{C}^{0}$ is totally epimorphic, and, moreover, an object of $\mathcal{C}^{0}$ is connected [as an object of $\mathcal{C}^{0}$ !] if and only if [cf. the argument of the preceding paragraph!] it is mobile [as an object of $\mathcal{C}^{0}$; in particular, [assuming still that $\mathcal{C}$ is almost totally epimorphic] every object of $\mathcal{C}^{0}$ is quasi-connected [as an object of $\left.\mathcal{C}^{0}\right]$. 
If $\mathcal{C}$ is a category, then we shall write

$$
\left.\mathcal{C}^{\perp} \text { (respectively, } \mathcal{C}^{\top}\right)
$$

for the category formed from $\mathcal{C}$ by taking arbitrary "formal" [possibly empty] finite (respectively, countables) coproducts of objects in $\mathcal{C}$. That is to say, we define the "Hom" of $\mathcal{C}^{\perp}$ (respectively, $\mathcal{C}^{\top}$ ) by the following formula:

$$
\operatorname{Hom}\left(\coprod_{i} A_{i}, \coprod_{j} B_{j}\right) \stackrel{\text { def }}{=} \prod_{i} \coprod_{j} \operatorname{Hom}_{\mathcal{C}}\left(A_{i}, B_{j}\right)
$$

[where the $A_{i}, B_{j}$ are objects of $\mathcal{C}$ ]. Thus, $\mathcal{C}^{\perp}$ (respectively, $\mathcal{C}^{\top}$ ) is a category of finitely (respectively, countably) connected type. Note that objects of $\mathcal{C}$ define connected objects of $\mathcal{C}^{\perp}$ or $\mathcal{C}^{\top}$. Moreover, there are natural [up to isomorphism] equivalences of categories

$$
\left(\mathcal{C}^{\perp}\right)^{0} \stackrel{\sim}{\rightarrow} \mathcal{C} ; \quad\left(\mathcal{C}^{\top}\right)^{0} \stackrel{\sim}{\rightarrow} \mathcal{C} ; \quad\left(\mathcal{D}^{0}\right)^{\perp} \stackrel{\sim}{\rightarrow} \mathcal{D} ; \quad\left(\mathcal{E}^{0}\right)^{\top} \stackrel{\sim}{\rightarrow} \mathcal{E}
$$

if $\mathcal{D}$ (respectively, $\mathcal{E}$ ) is a category of finitely connected type (respectively, category of countably connected type). If $\mathcal{C}$ is a totally epimorphic category of quasi-connected objects, then $\mathcal{C}^{\perp}$ (respectively, $\mathcal{C}^{\top}$ ) is an almost totally epimorphic category of finitely (respectively, countably) connected type.

In particular, the operations "0", " $\perp$ " (respectively, "T") define one-to-one correspondences [up to equivalence] between the totally epimorphic categories of quasi-connected objects and the almost totally epimorphic categories of finitely (respectively, countably) connected type.

If $\mathcal{C}$ is a $[$ small $]$ category, then we shall write $\mathbb{G}(\mathcal{C})$ for the graph associated to $\mathcal{C}$. This graph is the graph with precisely one vertex for each object of $\mathcal{C}$ and precisely one edge for each arrow of $\mathcal{C}$ [joining the vertices corresponding to the domain and codomain of the arrow]. We shall refer to the full subcategory of $\mathcal{C}$ determined by the objects and arrows that compose a connected component of the graph $\mathbb{G}(\mathcal{C})$ as a connected component of $\mathcal{C}$. In particular, we shall say that $\mathcal{C}$ is connected if $\mathbb{G}(\mathcal{C})$ is connected. [Note that by working with respect to some "sufficiently large" envelopping universe, it makes sense to speak of a category which is not necessarily small as being connected.]

If $\mathcal{C}$ is a category, then we shall say that an object $A \in \mathrm{Ob}(\mathcal{C})$ is indissectible if, for every pair of arrows $A_{1} \rightarrow A, A_{2} \rightarrow A$ of $\mathcal{C}$, where $A_{1}, A_{2}$ are nonempty, there exists a pair of arrows $\psi_{1}: B \rightarrow A_{1}, \psi_{2}: B \rightarrow A_{2}$ such that $\phi_{1} \circ \psi_{1}=$ $\phi_{2} \circ \psi_{2}$, where $B$ is nonempty.

If $\mathcal{C}$ if a category and $\mathcal{S}$ is a collection of arrows in $\mathcal{C}$, then we shall say that an arrow $A \rightarrow B$ is minimal-adjoint to $\mathcal{S}$ if every factorization $A \rightarrow C \rightarrow B$ of 
this arrow $A \rightarrow B$ in $\mathcal{C}$ such that $A \rightarrow C$ lies in $\mathcal{S}$ satisfies the property that $A \rightarrow C$ is, in fact, an isomorphism. Often, the collection $\mathcal{S}$ will be taken to be the collection of arrows satisfying a particular property $\mathcal{P}$; in this case, we shall refer to the property of being "minimal-adjoint to $\mathcal{S}$ " as the minimal-adjoint notion to $\mathcal{P}$.

\section{Curves}

Suppose that $g \geq 0$ is an integer. Then if $S$ is a scheme, a family of curves of genus $g$

$$
X \rightarrow S
$$

is defined to be a smooth, proper, geometrically connected morphism of schemes $X \rightarrow S$ whose geometric fibers are curves of genus $g$.

Suppose that $g, r \geq 0$ are integers such that $2 g-2+r>0$. We shall denote the moduli stack of $r$-pointed stable curves of genus $g$ (where we assume the points to be unordered) by $\overline{\mathcal{M}}_{g, r}[\mathrm{cf}$. [DM], [Knud] for an exposition of the theory of such curves; strictly speaking, [Knud] treats the finite étale covering of $\overline{\mathcal{M}}_{g, r}$ determined by ordering the marked points]. The open substack $\mathcal{M}_{g, r} \subseteq \overline{\mathcal{M}}_{g, r}$ of smooth curves will be referred to as the moduli stack of smooth r-pointed stable curves of genus $g$ or, alternatively, as the moduli stack of hyperbolic curves of type $(g, r)$. The divisor at infinity $\overline{\mathcal{M}}_{g, r} \backslash \mathcal{M}_{g, r}$ of $\overline{\mathcal{M}}_{g, r}$ determines a $\log$ structure on $\overline{\mathcal{M}}_{g, r}$; denote the resulting log stack by $\overline{\mathcal{M}}_{g, r}^{\log }$.

A family of hyperbolic curves of type $(g, r)$

$$
X \rightarrow S
$$

is defined to be a morphism which factors $X \hookrightarrow Y \rightarrow S$ as the composite of an open immersion $X \hookrightarrow Y$ onto the complement $Y \backslash D$ of a relative divisor $D \subseteq Y$ which is finite étale over $S$ of relative degree $r$, and a family $Y \rightarrow S$ of curves of genus $g$. One checks easily that, if $S$ is normal, then the pair $(Y, D)$ is unique up to canonical isomorphism. (Indeed, when $S$ is the spectrum of a field, this fact is well-known from the elementary theory of algebraic curves. Next, we consider an arbitrary connected normal $S$ on which a prime $l$ is invertible (which, by Zariski localization, we may assume without loss of generality). Denote by $S^{\prime} \rightarrow S$ the finite étale covering parametrizing orderings of the marked points and trivializations of the l-torsion points of the Jacobian of $Y$. Note that $S^{\prime} \rightarrow S$ is independent of the choice of $(Y, D)$, since (by the normality of $S$ ), $S^{\prime}$ may be constructed as the normalization of $S$ in the function field of $S^{\prime}$ (which is independent of the choice of $(Y, D)$ since 
the restriction of $(Y, D)$ to the generic point of $S$ has already been shown to be unique). Thus, the uniqueness of $(Y, D)$ follows by considering the classifying morphism (associated to $(Y, D)$ ) from $S^{\prime}$ to the finite étale covering of $\left(\mathcal{M}_{g, r}\right)_{\mathbb{Z}\left[\frac{1}{\tau}\right]}$ parametrizing orderings of the marked points and trivializations of the $l$-torsion points of the Jacobian [since this covering is well-known to be a scheme, for $l$ sufficiently large].) We shall refer to $Y$ (respectively, $D ; D ; D$ ) as the compactification (respectively, divisor at infinity; divisor of cusps; divisor of marked points) of $X$. A family of hyperbolic curves $X \rightarrow S$ is defined to be a morphism $X \rightarrow S$ such that the restriction of this morphism to each connected component of $S$ is a family of hyperbolic curves of type $(g, r)$ for some integers $(g, r)$ as above.

Write

$$
\overline{\mathcal{C}}_{g, r} \rightarrow \overline{\mathcal{M}}_{g, r}
$$

for the tautological curve over $\overline{\mathcal{M}}_{g, r} ; \overline{\mathcal{D}}_{g, r} \subseteq \overline{\mathcal{M}}_{g, r}$ for the corresponding tautological divisor of marked points. The divisor given by the union of $\overline{\mathcal{D}}_{g, r}$ with the inverse image in $\overline{\mathcal{C}}_{g, r}$ of the divisor at infinity of $\overline{\mathcal{M}}_{g, r}$ determines a $\log$ structure on $\overline{\mathcal{C}}_{g, r}$; denote the resulting $\log$ stack by $\overline{\mathcal{C}}_{g, r}^{\log }$. Thus, we obtain a morphism of log stacks

$$
\overline{\mathcal{C}}_{g, r}^{\log } \rightarrow \overline{\mathcal{M}}_{g, r}^{\log }
$$

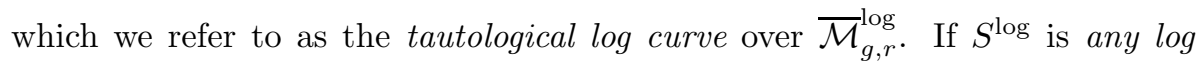
scheme, then we shall refer to a morphism

$$
C^{\log } \rightarrow S^{\log }
$$

which is obtained as the pull-back of the tautological log curve via some [necessarily uniquely determined - cf., e.g., [Mzk1], §3] classifying morphism $S^{\log } \rightarrow$ $\overline{\mathcal{M}}_{g, r}^{\log }$ as a stable log curve. If $C$ has no nodes, then we shall refer to $C^{\log } \rightarrow S^{\log }$ as a smooth log curve.

If $X_{K}$ (respectively, $Y_{L}$ ) is a hyperbolic curve over a field $K$ (respectively, $L$ ), then we shall say that $X_{K}$ is isogenous to $Y_{L}$ if there exists a hyperbolic curve $Z_{M}$ over a field $M$ together with finite étale morphisms $Z_{M} \rightarrow X_{K}$, $Z_{M} \rightarrow Y_{L}$.

\section{$\S 1$. Zariski's Main Theorem for Semi-graphs}

In this $\S$, we prove an analogue [cf. Theorem 1.2 below] for semi-graphs of "Zariski's main theorem" (for schemes). 
We begin with some general remarks concerning semi-graphs [a notion defined in [Mzk3], Appendix]. First, we recall that a semi-graph $\mathbb{G}$ consists of the following collection of data:

(1) a set $\mathcal{V}$ - whose elements we refer to as "vertices";

(2) a set $\mathcal{E}$ - whose elements we refer to as "edges" — each of whose elements $e$ is a set of cardinality 2 satisfying the property " $e \neq e^{\prime} \in \mathcal{E} \Longrightarrow e \bigcap e^{\prime}=\emptyset$ ";

(3) a collection $\zeta$ of maps $\zeta_{e}$ [one for each edge $e$ ] — which we refer to as the "coincidence maps" — such that $\zeta_{e}: e \rightarrow \mathcal{V} \bigcup\{\mathcal{V}\}$ [where we note that $\mathcal{V} \cap\{\mathcal{V}\}=\emptyset$ since $\mathcal{V} \notin \mathcal{V}]$ is a map from the set $e$ to the set $\mathcal{V} \cup\{\mathcal{V}\}$.

We shall refer to the subset $\zeta_{e}^{-1}(\mathcal{V}) \subseteq e$ [i.e., the inverse image of the subset $\mathcal{V} \subseteq \mathcal{V} \bigcup\{\mathcal{V}\}$ of elements $\neq \mathcal{V}]$ as the verticial portion of an edge $e$; to the restriction of $\zeta_{e}$ to the verticial portion of $e$ as the verticial restriction of $\zeta_{e}$; and to the cardinality of the verticial portion of $e$ as the verticial cardinality of e. A graph $\mathbb{G}$ is a semi-graph $\mathbb{G}$ for which every $e \in \mathcal{E}$ has verticial cardinality precisely 2 . We shall refer to an element $b \in e$ as a branch of the edge $e$. A semigraph will be called finite (respectively, countable) if both its set of vertices and its set of edges are finite (respectively, countable). A component of a semi-graph is defined to be the datum of either an edge or a vertex of the semi-graph.

Let $\mathbb{G}=\{\mathcal{V}, \mathcal{E}, \zeta\}$ be a semi-graph. If $e \in \mathcal{E}$ is an edge of $\mathbb{G}$ of verticial cardinality 2 whose image via $\zeta_{e}$ consists of (not necessarily distinct) elements $v_{1}, v_{2}$ of $\mathcal{V}$, then we shall say that $e$ joins $v_{1}$ to $v_{2}$. If $v=\zeta_{e}(b)$, for some branch $b$ of an edge $e$ [so $v$ is a vertex], then we shall say that the edge $e$ meets or abuts to the vertex $v$, and that the branch $b$ of the edge $e$ abuts to the vertex $v$. Thus, an edge of a graph always abuts to at least one vertex, while an edge of a semi-graph may abut to no vertices at all. A morphism between semi-graphs

$$
\mathbb{G}=\{\mathcal{V}, \mathcal{E}, \zeta\} \rightarrow \mathbb{G}^{\prime}=\left\{\mathcal{V}^{\prime}, \mathcal{E}^{\prime}, \zeta^{\prime}\right\}
$$

is a collection of maps $\mathcal{V} \rightarrow \mathcal{V}^{\prime} ; \mathcal{E} \rightarrow \mathcal{E}^{\prime}$; and for each $e \in \mathcal{E}$ mapping to $e^{\prime}$, a bijection $e \stackrel{\sim}{\rightarrow} e^{\prime}$ [or, equivalently — since both $e$ and $e^{\prime}$ are sets of cardinality 2 - an injection $\left.e \hookrightarrow e^{\prime}\right]$ - all of which are compatible with the verticial restrictions of the respective coincidence maps. Thus, here, we allow an edge that abuts to no (respectively, precisely one) vertex to map to an edge that abuts to any number $\geq 0$ (respectively, $\geq 1$ ) of vertices.

A semi-graph $\mathbb{G}$ may be thought of as a topological space as follows: We regard each vertex $v$ as a point $[v]$. If $e$ is an edge, consisting of branches $b_{1}, b_{2}$, then we regard $e$ as the "interval" given by the set of formal sums 


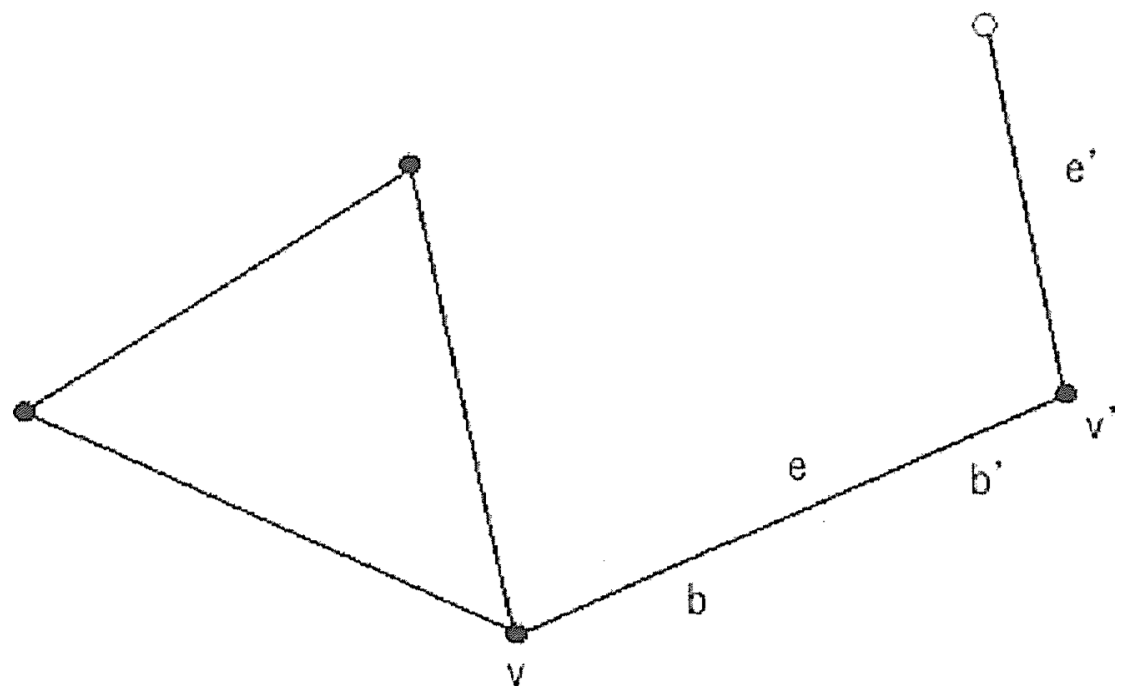

A Typical Semi-graph

$v, v^{\prime}$ : vertices; $\quad e$ : a closed edge; $\quad e^{\prime}$ : an open edge that abuts to $v^{\prime}$ $b$ : a branch of $e$ that abuts to $v ; \quad b^{\prime}$ : a branch of $e$ that abuts to $v^{\prime}$

$\lambda_{1} \cdot\left[b_{1}\right]+\lambda_{2} \cdot\left[b_{2}\right]$, where $\lambda_{1}, \lambda_{2} \in \mathbb{R}$ [here, $\mathbb{R}$ denotes the topological field of real numbers]; $\lambda_{1}+\lambda_{2}=1$; for $i=1,2, \lambda_{i} \leq 1$ (respectively, $\lambda_{i}<1$ ) if $b_{i}$ abuts (respectively, does not abut) to a vertex; moreover, if $b_{i}$ abuts to a vertex $v$, then we identify the formal sum $1 \cdot\left[b_{i}\right]+0 \cdot\left[b_{3-i}\right]$ with $[v]$. Thus, relative to this point of view, it is natural to think of the branch $b_{i}$ as the portion of the interval just defined consisting of formal sums such that $\lambda_{i}>\frac{1}{2}$. Also, we observe that this construction of an associated topological space is functorial: Every morphism of semi-graphs induces a continuous morphism of the corresponding topological spaces. In the following discussion, we shall often invoke this point of view without further explanation.

A sub-semi-graph $\mathbb{H}$ of a semi-graph $\mathbb{G}$ is a semi-graph satisfying the following properties: (a) the set of vertices (respectively, edges) of $\mathbb{H}$ is a subset of the set of vertices (respectively, edges) of $\mathbb{G}$; (b) every branch of an edge of $\mathbb{H}$ that abuts, relative to $\mathbb{G}$, to a vertex $v$ of $\mathbb{G}$ lying in $\mathbb{H}$ also abuts to $v$, relative to $\mathbb{H}$; (c) if a branch of an edge of $\mathbb{H}$ either abuts, relative to $\mathbb{G}$, to a vertex $v$ of $\mathbb{G}$ that does not lie in $\mathbb{H}$, or does not abut to a vertex, relative to $\mathbb{G}$, then this branch does not abut to a vertex, relative to $\mathbb{H}$. A morphism of semi-graphs will be called an embedding if it induces an isomorphism of the domain onto a sub-semi-graph of the codomain. 
Let $\mathbb{G}$ be a semi-graph. Then we shall refer to an edge of $\mathbb{G}$ that is of verticial cardinality 2 (respectively, $<2 ; 0)$ as closed (respectively, open; isolated). We shall say that two closed edges $e$ and $e^{\prime}$ of $\mathbb{G}$ are coverticial if the following condition holds: the edge $e$ abuts to a vertex $v$ of $\mathbb{G}$ if and only if the edge $e^{\prime}$ abuts to $v$. We shall say that $\mathbb{G}$ is locally finite if, for every vertex $v$ of $\mathbb{G}$, the set of edges that abut to $v$ is finite. We shall say that $\mathbb{G}$ is untangled if every closed edge of $\mathbb{G}$ abuts to two distinct vertices. We shall refer to a connected semi-graph that has precisely one vertex and precisely two edges, both of which are open, as a joint. If a sub-semi-graph of a given semi-graph is a joint, then we shall refer to this sub-semi-graph as a subjoint of the given semi-graph. We shall refer to the sub-semi-graph of $\mathbb{G}$ obtained by omitting all of the open edges as the maximal subgraph of the semi-graph. We shall refer to as the compactification of $\mathbb{G}$ the graph obtained from $\mathbb{G}$ by appending to $\mathbb{G}$, for each branch $b$ of an edge of $\mathbb{G}$ that does not abut to a vertex, a new vertex $v_{b}$ to which $b$ is to abut. Thus, $\mathbb{G}$ forms a sub-semi-graph of its compactification. Moreover, any morphism of semi-graphs induces a unique morphism between the respective compactifications. Finally, we observe that every connected component of the topological space associated to the maximal subgraph of $\mathbb{G}$ (respectively, $\mathbb{G}$ ) is a deformation retract [in the sense of algebraic topology] of the corresponding connected component of the topological space associated to $\mathbb{G}$ (respectively, the compactification of $\mathbb{G}$ ). A semi-graph whose associated topological space is contractible [in the sense of algebraic topology] will be referred to as a tree.

We recall in passing that there is a semi-graph that is naturally associated to any pointed stable curve over an algebraically closed field [cf. [Mzk3], Appendix]: That is to say, the vertices (respectively, closed edges; open edges; branches of a closed edge) of this semi-graph are precisely the irreducible components (respectively, nodes; marked points; branches of a node) of the pointed stable curve. The coincidence maps are determined in an evident fashion by the geometry of the pointed stable curve.

Let $v$ (respectively, $e ; b)$ be a(n) vertex (respectively, edge; branch of an edge) of $\mathbb{G}$. Then we define morphisms of semi-graphs

$$
\mathbb{G}[v] \rightarrow \mathbb{G} ; \quad \mathbb{G}[e] \rightarrow \mathbb{G} ; \quad \mathbb{G}[b] \rightarrow \mathbb{G}
$$

as follows: $\mathbb{G}[v]$ consists of a single vertex $v^{\prime}$, which maps to $v$, and, for each branch $b_{v}$ of an edge $e_{v}$ of $\mathbb{G}$ that abuts to $v$, an edge $e_{b_{v}}^{\prime}$ of verticial cardinality 1 that maps to $e_{v}$ in such a way that the branch of $e_{b_{v}}^{\prime}$ lying over $b_{v}$ abuts to $v^{\prime}$. $\mathbb{G}[e]$ consists of a single edge $e^{\prime}$, which maps to $e$, and, for each branch $b_{e}$ of $e$ abutting to a vertex $v_{b_{e}}$ of $\mathbb{G}$, a vertex $v_{b_{e}}^{\prime}$ [of $\left.\mathbb{G}[e]\right]$ that maps to $v_{b_{e}}$ and is the abutment of the branch $b_{e^{\prime}}^{\prime}$ of $e^{\prime}$ that lies over $b_{e}$. If $b$ is a branch of 
an edge $e_{b}$ that abuts to a vertex $v_{b}[\mathrm{of} \mathbb{G}]$, then $\mathbb{G}[b]$ is the sub-semi-graph of $\mathbb{G}\left[e_{b}\right]$ consisting of the unique edge of $\mathbb{G}\left[e_{b}\right]$ and the vertex of $\mathbb{G}\left[e_{b}\right]$ which is the abutment of the branch of this unique edge that lies over $b$. Thus, $\mathbb{G}[v], \mathbb{G}[e]$, $\mathbb{G}[b]$ are all trees [even if $\mathbb{G}$ fails to be untangled]; if the branch $b$ is a branch of the edge $e$ that abuts to $v$, then we have natural morphisms $\mathbb{G}[b] \rightarrow \mathbb{G}[v]$, $\mathbb{G}[b] \rightarrow \mathbb{G}[e]$ over $\mathbb{G}$.

A morphism

$$
\phi: \mathbb{G}_{A} \rightarrow \mathbb{G}_{B}
$$

between semi-graphs will be called an immersion [or an immersive morphism] (respectively, excision [or an excisive morphism]) if it satisfies the condition that, for every vertex $v_{A}$ of $\mathbb{G}_{A}$ that maps to a vertex $v_{B}$ of $\mathbb{G}_{B}$, the induced map from branches abutting to $v_{A}$ to branches abutting to $v_{B}$ is injective (respectively, bijective). Thus, if we think of $\mathbb{G}_{A}$ and $\mathbb{G}_{B}$ as topological spaces, then an immersion $\phi: \mathbb{G}_{A} \rightarrow \mathbb{G}_{B}$ is locally [in some small neighborhood of every point of $\mathbb{G}_{A}$ ] an embedding (respectively, a homeomorphism) of topological spaces.

Observe that: the five classes of morphisms $\mathbb{G}[v] \rightarrow \mathbb{G}, \mathbb{G}[b] \rightarrow \mathbb{G}[e], \mathbb{G}[e] \rightarrow$ $\mathbb{G}, \mathbb{G}[b] \rightarrow \mathbb{G}, \mathbb{G}[b] \rightarrow \mathbb{G}[v]$, are all immersive; the first two of these classes are always excisive; the last three of these classes are not excisive in general.

Also, we observe that a morphism of sub-semi-graphs $\mathbb{G}_{A} \subseteq \mathbb{G}_{B}$ is immersive (respectively, excisive) if and only if, for every vertex $v_{A}$ of $\mathbb{G}_{A}$ mapping to a vertex $v_{B}$ of $\mathbb{G}_{B}$, the induced morphism of semi-graphs $\mathbb{G}_{A}\left[v_{A}\right] \rightarrow \mathbb{G}_{B}\left[v_{B}\right]$ is an embedding (respectively, isomorphism).

A morphism of semi-graphs

$$
\phi: \mathbb{G}_{A} \rightarrow \mathbb{G}_{B}
$$

will be called proper if it preserves verticial cardinalities of edges. A proper excision will be referred to as a graph-covering. A graph-covering with finite fibers will be referred to as a finite graph-covering. Note that if $\phi: \mathbb{G}_{A} \rightarrow \mathbb{G}_{B}$ is a graph-covering, with $\mathbb{G}_{A}, \mathbb{G}_{B}$ connected, then the associated map of topological spaces will be a covering in the sense of algebraic topology. Conversely, every covering, in the sense of algebraic topology, of the topological space associated to $\mathbb{G}_{B}$ arises in this way. Also, we observe that, just as in the case of coverings of topological spaces, it makes sense to speak of a graph-covering as Galois [i.e., "arising from a normal subgroup of the fundamental group"] and to speak of the pull-back of a graph-covering by an arbitrary morphism of semi-graphs.

Proposition 1.1. Any immersion from a connected graph into a tree is, in fact, an embedding. 
Proof. Indeed, suppose that we are given an immersion $\phi: \mathbb{G}_{A} \rightarrow \mathbb{G}_{B}$ into a tree $\mathbb{G}_{B}$ which is not an embedding. If $\phi$ is injective on vertices, then it follows from the definition of an immersion that $\phi$ is injective on edges, hence that $\phi$ is an embedding. Thus, it suffices to show that $\phi$ is injective on vertices.

Suppose that there exist distinct vertices $v_{1}, v_{2}$ of $\mathbb{G}_{A}$ that map to the same vertex $w$ of $\mathbb{G}_{B}$. Write $\gamma_{A}$ for a path on $\mathbb{G}_{A}$ that connects $v_{1}$ to $v_{2}$. Without loss of generality, we may assume that $\gamma_{A}$ has minimal length among paths on $\mathbb{G}_{A}$ that join distinct vertices of $\mathbb{G}_{A}$ that map to the same vertex of $\mathbb{G}_{B}$. Write $\gamma_{B} \stackrel{\text { def }}{=} \phi\left(\gamma_{A}\right)$. Then note that the minimality condition (together with the fact that $\phi$ is an immersion) implies that $\gamma_{B}$ does not intersect itself. Thus, $\gamma_{B}$ is a loop, starting and ending at $w$, and defined by a sequence of edges, all of which are distinct. But this contradicts the fact that $\mathbb{G}_{B}$ is a tree. This completes the proof.

Thus, in particular, if we start with an arbitrary immersion of connected graphs [which are not necessarily trees]

$$
\phi: \mathbb{G}_{A} \rightarrow \mathbb{G}_{B}
$$

then Proposition 1.1 implies that the induced morphism

$$
\widetilde{\mathbb{G}}_{A} \rightarrow \widetilde{\mathbb{G}}_{B}
$$

on universal graph-coverings [i.e., the associated topological coverings are universal coverings of $\mathbb{G}_{A}, \mathbb{G}_{B}$, respectively, in the sense of algebraic topology] - which are well-defined up to composition with deck transformations - is an embedding [since it is an immersion into a tree]. More generally, given an arbitrary graph-covering

$$
\mathbb{G}_{B^{\prime}} \rightarrow \mathbb{G}_{B}
$$

one can ask when the base-changed immersion

$$
\phi^{\prime}: \mathbb{G}_{A^{\prime}} \rightarrow \mathbb{G}_{B^{\prime}}
$$

is an embedding on each connected component of $\mathbb{G}_{A^{\prime}}$. Proposition $1.1 \mathrm{im-}$ plies that the universal graph-covering $\widetilde{\mathbb{G}}_{B} \rightarrow \mathbb{G}_{B}$ is sufficient to realize this condition.

In fact, however, when $\mathbb{G}_{A}, \mathbb{G}_{B}$ are finite, this condition may be realized by a finite graph-covering $\mathbb{G}_{B^{\prime}} \rightarrow \mathbb{G}_{B}$ :

Theorem 1.2 ("Zariski's Main Theorem for Semi-graphs"). Let

$$
\phi: \mathbb{G}_{A} \rightarrow \mathbb{G}_{B}
$$

be an immersion of finite semi-graphs. Then: 
(i) The morphism $\phi$ factors as the composite of an embedding

$$
\mathbb{G}_{A} \hookrightarrow \mathbb{G}_{B^{\prime}}
$$

and $a$ finite graph-covering $\mathbb{G}_{B^{\prime}} \rightarrow \mathbb{G}_{B}$.

(ii) There exists a finite graph-covering $\mathbb{G}_{B^{\prime}} \rightarrow \mathbb{G}_{B}$ such that the restriction of the base-changed morphism

$$
\phi^{\prime}: \mathbb{G}_{A^{\prime}} \rightarrow \mathbb{G}_{B^{\prime}}
$$

to each connected component of $\mathbb{G}_{A^{\prime}}$ is an embedding.

Remark 1.2.1. The author is indebted to M. Matsumoto for the following elegant graph-theoretic proof of Theorem 1.2.

Remark 1.2.2. The general form of Theorem 1.2 is reminiscent of the well-known result in algebraic geometry ("Zariski's Main Theorem" — cf., e.g., [Milne], Chapter I, Theorem 1.8) that any separated quasi-finite morphism

$$
f: X \rightarrow Y
$$

between noetherian schemes factors as the composite of an open immersion $X \hookrightarrow Y^{\prime}$ and a finite morphism $Y^{\prime} \rightarrow Y-$ cf. also Lemma 1.5 below.

Proof. First, we observe that (ii) follows formally from (i) [by taking the finite graph-covering of (ii) to be a Galois finite graph-covering of $\mathbb{G}_{B}$ that dominates the graph-covering of (i)]. Thus, it suffices to prove (i).

Next, let us observe that:

(a) Any immersion of semi-graphs for which the induced morphism between the respective compactifications is an embedding is itself an embedding (of semi-graphs).

(b) Restriction from the compactification of $\mathbb{G}_{B}$ to $\mathbb{G}_{B}$ induces an equivalence of categories between the respective categories of finite graph-coverings. Moreover, the compactification of a finite graph-covering of $\mathbb{G}_{B}$ is naturally isomorphic to the corresponding finite graph-covering of the compactification of $\mathbb{G}_{B}$.

In particular, by replacing the semi-graphs involved by their compactifications, it suffices to prove (i) in the case where all of the semi-graphs are, in fact, 
graphs. Thus, for the remainder of the proof, we assume that $\mathbb{G}_{A}, \mathbb{G}_{B}$ are graphs.

Let us write

$$
\mathbb{H}_{n}
$$

(where $n \geq 1$ is an integer) for the graph consisting of one vertex $v_{\mathbb{H}}$ and $n$ edges $e_{\mathbb{H}, 1} ; \ldots ; e_{\mathbb{H}, n}$ (all of which run from $v_{\mathbb{H}}$ to $v_{\mathbb{H}}$ ).

Next, let us observe that by Lemma 1.4 below, there exists an immersion

$$
\zeta: \mathbb{G}_{B} \rightarrow \mathbb{H}_{n}
$$

which we may compose with $\phi$ to form an immersion:

$$
\psi: \mathbb{G}_{A} \rightarrow \mathbb{H}_{n}
$$

Moreover, since pull-backs of finite graph-coverings of $\mathbb{H}_{n}$ via $\zeta$ form finite graph-coverings of $\mathbb{G}_{B}$, it follows that in order to prove that the assertion of Theorem 1.2, (i), is true for $\phi$, it suffices to prove that it is true for $\psi$. On the other hand, Theorem 1.2, (i), follows for $\psi$ by Lemma 1.5 below.

Note that, relative to the topological space point of view discussed above, the vertex $v_{\mathbb{H}}$ of the graph $\mathbb{H}_{n}$ meets precisely $2 n$ branches.

Lemma 1.3. Let $\mathbb{G}$ be a finite graph. Then:

(i) To give a morphism

$$
\phi: \mathbb{G} \rightarrow \mathbb{H}_{n}
$$

is equivalent to assigning an orientation and a "color" $\in\{1, \ldots, n\}$ to each edge of $\mathbb{G}$.

(ii) The morphism $\phi$ is an immersion if and only if for each color $i \in$ $\{1, \ldots, n\}$, and at each vertex $v$ of $\mathbb{G}$, the number of branches of color $i$ that enter (respectively, leave) $v$ - i.e., relative to the assigned orientations - is $\leq 1$.

(iii) The morphism $\phi$ is an excision [or, equivalently, a finite graphcovering] if and only if for each color $i \in\{1, \ldots, n\}$, and at each vertex $v$ of $\mathbb{G}$, the number of branches of color $i$ that enter (respectively, leave) $v-i . e$, relative to the assigned orientations - is $=1$.

Proof. $\quad$ First, we fix an orientation on each edge $e_{\mathbb{H}, i}$ of $\mathbb{H}_{n}$, and regard the edge $e_{\mathbb{H}, i}$ as being of color $i$. 
Now let us prove (i). Given a morphism $\phi: \mathbb{G} \rightarrow \mathbb{H}_{n}$, we obtain orientations and colors on the edges of $\mathbb{G}$ by pulling back the orientations and colors of $\mathbb{H}_{n}$ via $\phi$. Conversely, given a choice of orientations and colors on the edges of $\mathbb{G}$, we obtain a morphism $\phi: \mathbb{G} \rightarrow \mathbb{H}_{n}$ by sending all the vertices of $\mathbb{G}$ to $v_{\mathbb{H}}$ and mapping the edges of $\mathbb{G}$ to the edges of $\mathbb{H}_{n}$ in the unique way which preserves orientations and colors.

Assertions (ii) and (iii) follow immediately by considering the local structure of $\mathbb{H}_{n}$ at $v_{\mathbb{H}}$. Note that in general, a morphism of finite graphs is always proper, hence is a finite graph-covering if and only if it is excisive.

Lemma 1.4. Every finite graph $\mathbb{G}$ admits an immersion $\mathbb{G} \rightarrow \mathbb{H}_{n}$ for some integer $n \geq 1$.

Proof. Indeed, if we take $n$ to be the number of edges of $\mathbb{G}$ and assign distinct colors to distinct edges of $\mathbb{G}$, then it is immediate from Lemma 1.3, (ii), that (for any assignment of orientations) the resulting morphism $\mathbb{G} \rightarrow \mathbb{H}_{n}$ is an immersion.

Lemma 1.5. Let $\phi: \mathbb{G} \rightarrow \mathbb{H}_{n}$ be an immersion of finite graphs. Then $\phi$ extends to a finite graph-covering $\phi^{\prime}: \mathbb{G}^{\prime} \rightarrow \mathbb{H}_{n}$ for some embedding $\mathbb{G} \hookrightarrow \mathbb{G}^{\prime}$.

Proof. We construct $\left(\mathbb{G}^{\prime}, \phi^{\prime}\right)$ from $(\mathbb{G}, \phi)$ by adding edges (equipped with orientations and colors) to $\mathbb{G}$ until the resulting $\phi^{\prime}$ is excisive, i.e., satisfies the condition of Lemma 1.3, (iii). Suppose that there exists a vertex $v$ of $\mathbb{G}$ that does not satisfy this condition. This means that there is some color $i$ such that either there does not exist a branch of color $i$ entering $v$ or there does not exist a branch of color $i$ leaving $v$ (or both). If there do not exist any branches of color $i$ meeting $v$, then we simply add an edge of color $i$ to $\mathbb{G}$ that runs from $v$ to $v$. Now suppose (without loss of generality) that there exists a branch of color $i$ leaving $v$, but that there does not exist a branch of color $i$ entering $v$. Then we follow the $i$-colored edge leaving $v_{1} \stackrel{\text { def }}{=} v$ to a new vertex $v_{2}$ (necessarily distinct from $v_{1}$ ). Now there are two possibilities:

(1) There exists an $i$-colored edge leaving this vertex.

(2) There does not exist an $i$-colored edge leaving this vertex.

If (1) holds, then we repeat the above procedure - i.e., we follow this $i$-colored edge out of $v_{2}$ to another vertex $v_{3}$, which is necessarily distinct from $v_{2}$ since 
the unique (by Lemma 1.3, (ii)) $i$-colored edge entering $v_{2}$ originated from a vertex which is distinct from $v_{2}$. Thus, continuing in this way, we obtain a sequence

$$
v_{1}, v_{2}, v_{3}, \ldots
$$

of distinct (by Lemma 1.3, (ii)) vertices of $\mathbb{G}$. Since $\mathbb{G}$ is finite, this sequence must eventually terminate at some vertex $v_{k}$ satisfying (2). Then we add an $i$-colored edge to $\mathbb{G}$ running from $v_{k}$ to $v_{1}$ to form a pair $(\mathbb{G}[2], \phi[2])$ extending the original $(\mathbb{G}[1], \phi[1]) \stackrel{\text { def }}{=}(\mathbb{G}, \phi)$.

Note that $\phi[2]$ is still an immersion, that $\mathbb{G}[2]$ has the same set of vertices as $\mathbb{G}[1]$, that the set of "colors" [labeled $1, \ldots, n]$ remains unchanged, and that the cardinality of the [finite] set of [ordered] pairs consisting of a vertex and a color which violate the condition of Lemma 1.3, (iii), relative to $\phi[2]$, is $<$ the cardinality of the [finite] set of [ordered] pairs consisting of a vertex and a color which violate the condition of Lemma 1.3, (iii), relative to $\phi[1]$. Thus, if we apply the procedure

$$
(\mathbb{G}[1], \phi[1]) \mapsto(\mathbb{G}[2], \phi[2])
$$

to $(\mathbb{G}[2], \phi[2])$ to obtain some $(\mathbb{G}[3], \phi[3])$, and so on, we obtain a sequence of pairs

$$
(\mathbb{G}[1], \phi[1]) ;(\mathbb{G}[2], \phi[2]) ;(\mathbb{G}[3], \phi[3]) ; \ldots
$$

which — by the finiteness of the sets of vertices and colors - necessarily terminates in a pair $\left(\mathbb{G}^{\prime}, \phi^{\prime}\right)$ such that $\phi^{\prime}$ is a finite graph-covering, as desired.

Remark 1.5.1. Consider the case of an immersion

$$
\phi: \mathbb{G} \rightarrow \mathbb{H}_{1}
$$

where $\mathbb{G}$ is a finite connected graph. Since the (topological) fundamental group of $\mathbb{H}_{1}$ is equal to $\mathbb{Z}$, the isomorphism class of a (connected) finite graph-covering $\mathbb{G}^{\prime} \rightarrow \mathbb{H}_{1}$ of $\mathbb{H}_{1}$ is determined by its degree $d$ (a positive integer) [in the sense of algebraic topology]. Then one can ask what conditions one must place on $d$ for the corresponding finite graph-covering to satisfy the property of Theorem 1.2 , (ii). In some sense, there are essentially two phenomena that may occur:

(1) The case where $\phi$ itself is a finite graph-covering, of degree $n$. In this case, the resulting condition on $d$ is nonarchimedean, i.e.:

$$
d \equiv 0 \quad(\bmod n)
$$


(2) The case where $\mathbb{G}$ consists of $n$ vertices $v_{1}, \ldots, v_{n}$, and precisely one edge joining $v_{j}$ to $v_{j+1}$, for $j=1, \ldots, n-1$ (and no other edges). In this case, the resulting condition on $d$ is archimedean, i.e.:

$$
d \geq n
$$

The above analysis suggests that there is some interesting arithmetic "hidden" in Theorem 1.2.

The following interesting consequence of Theorem 1.2 - which asserts, in effect, that finitely generated subgroups of finite rank [discrete] free groups admit bases with properties reminiscent of their abelian counterparts - was pointed out to the author by A. Tamagawa:

Corollary 1.6 (Finitely generated Subgroups of Finite Rank Free Groups). Let $F$ be a finitely generated subgroup of a free group $G$ of finite rank (so $F$ is also free of finite rank). Then:

(i) There exists an immersion of finite graphs $\phi: \mathbb{G}_{A} \rightarrow \mathbb{G}_{B}$ whose induced morphism on (topological) fundamental groups is isomorphic to the inclusion $F \hookrightarrow G$.

(ii) There exists a finite index subgroup $H \subseteq G$ such that $H$ contains $F$, and, moreover, there exists a set of free generators $\gamma_{1}, \ldots, \gamma_{r}$ of $H$ with the property that for some $s \leq r, \gamma_{1}, \ldots, \gamma_{s}$ form a set of free generators of $F$.

Proof. $\quad$ First, observe that if $\mathbb{G}_{A} \hookrightarrow \mathbb{G}_{B^{\prime}}$ is an embedding, then any set of free generators of the fundamental group of $\mathbb{G}_{A}$ may be extended to a set of free generators of the fundamental group of $\mathbb{G}_{B^{\prime}}$. In light of this observation, assertion (ii) follows by applying Theorem 1.2, (i), to an immersion as in assertion (i) (of the present Corollary).

Thus, it suffices to prove (i). Let $\mathbb{G}_{B}$ be any graph whose fundamental group is equal to $G$. Then the subgroup $F \subseteq G$ defines an infinite graphcovering

$$
\mathbb{G}_{A^{\prime}} \rightarrow \mathbb{G}_{B}
$$

of $\mathbb{G}_{B}$. In particular, $\mathbb{G}_{A^{\prime}}$ has fundamental group equal to $F$. Although, in general, the graph $\mathbb{G}_{A^{\prime}}$ will not necessarily be finite, it follows from the fact that its fundamental group $F$ is finitely generated that there exists a finite subgraph $\mathbb{G}_{A} \subseteq \mathbb{G}_{A^{\prime}}$ such that the natural injection of fundamental groups $\pi_{1}\left(\mathbb{G}_{A}\right) \hookrightarrow$ $\pi_{1}\left(\mathbb{G}_{A^{\prime}}\right)$ is, in fact, a bijection. Moreover, the composite $\mathbb{G}_{A} \hookrightarrow \mathbb{G}_{A^{\prime}} \rightarrow \mathbb{G}_{B}$ is 
an immersion (since it is a composite of immersions). This completes the proof of (i).

Another interesting consequence of Theorem 1.2 is the following wellknown result:

Corollary 1.7 (Residual Finiteness of Free Groups). Every discrete free group $F$ injects into its profinite completion.

Proof. Indeed, let $\mathbb{G}$ be a connected graph with $\pi_{1}(\mathbb{G})=F$. If $H \subseteq$ $F$ is the kernel of the map from $F$ to its profinite completion, write $\mathbb{H} \rightarrow$ $\mathbb{G}$ for the corresponding graph-covering. If $\mathbb{H}$ is not a tree, then one verifies immediately that $\mathbb{H}$ contains a finite connected subgraph $\mathbb{H}^{\prime}$ which is not a tree. In particular, $\mathbb{H}^{\prime}$ admits nontrivial finite graph-coverings. Let $\mathbb{G}^{\prime}$ be a finite connected subgraph of $\mathbb{G}$ which contains the image of $\mathbb{H}^{\prime}$. Then if we apply Theorem 1.2, (i), to the immersion $\mathbb{H}^{\prime} \rightarrow \mathbb{G}^{\prime}$, we obtain [since finite graph-coverings of a subgraph of a given graph always extend to finite graphcoverings of the given graph] that there exists a finite graph-covering $\mathbb{K}^{\prime} \rightarrow \mathbb{G}^{\prime}$ whose pull-back to $\mathbb{H}^{\prime}$ is nontrivial. Thus, if we extend $\mathbb{K}^{\prime} \rightarrow \mathbb{G}^{\prime}$ to a finite graph-covering $\mathbb{K} \rightarrow \mathbb{G}$, we obtain a finite graph-covering of $\mathbb{G}$ whose pull-back to $\mathbb{H}$ is nontrivial. But this contradicts the definition of $H$.

Remark 1.7.1. We recall in passing that there is also a pro-l version of this residual finiteness result — cf., e.g., [RZ], Proposition 3.3.15.

Finally, before continuing, we note the following useful result concerning finite group actions on semi-graphs, which is implicit in the theory of [Serre]:

Lemma 1.8 (Finite Group Actions on Semi-graphs). Let $\mathbb{G}$ be a connected semi-graph, equipped with the action of a finite group $G$. Then:

(i) Every finite sub-semi-graph $\mathbb{G}^{\prime}$ of $\mathbb{G}$ is contained in a finite connected sub-semi-graph $\mathbb{G}^{\prime \prime}$ of $\mathbb{G}$ that is stabilized by the action of $G$.

(ii) Suppose that $\mathbb{G}$ is a tree. Then: (a) there exists at least one vertex or edge of $\mathbb{G}$ that is fixed by $G$; (b) if $G$ fixes two distinct vertices $w_{1}, w_{2}$ of $\mathbb{G}$, then $G$ acts trivially on any "geodesic" [i.e., path of closed edges of minimal length] that joins $w_{1}, w_{2} ;$ (c) if $G$ fixes three distinct vertices of $\mathbb{G}$, then there exists at least one subjoint of $\mathbb{G}$ on which $G$ acts trivially. 
Proof. First, we consider assertion (i). Since $\mathbb{G}$ is connected, we may assume without loss of generality that $\mathbb{G}^{\prime}$ is connected and contains the $G$-orbit of some vertex. Then one verifies easily that if we take $\mathbb{G}^{\prime \prime}$ to be the $G$-orbit of $\mathbb{G}^{\prime}$, then the desired properties are satisfied.

Next, we consider assertion (ii). First, we verify assertion (a). This follows formally from [Serre], Chapter I, §6.5, Corollary 3 to Proposition 26, Proposition 27 - at least if one assumes, as in done in [Serre], that $G$ fixes some orientation on the tree $\mathbb{G}$. On the other hand, by "splitting" each edge of $\mathbb{G}$ which violates this assumption into two new edges, corresponding to the two branches of the original edge, one sees immediately that one still obtains assertion (a), even without this assumption. This completes the proof of (ii), (a).

Next, to prove (ii), (b), recall from [Serre], Chapter I, §2.2, Proposition 8, that there is a unique path of minimal length from $w_{1}$ to $w_{2}$. Since $G$ fixes $w_{1}$, $w_{2}$, it thus follows that $G$ fixes this path. Thus, [since it is evident that there are no automorphisms of this path that fix $w_{1}, w_{2}$ ] we conclude that $G$ acts trivially on this path, as desired. This completes the proof of (ii), (b). Finally, we observe that (ii), (c) follows formally from (ii), (b).

Remark 1.8.1. We observe, in passing, that Lemma 1.8, (ii), (a), implies [the well-known fact — cf., e.g., [Serre], Chapter I, §3.4, Theorem 5] that a free group [which may be thought of as the fundamental group of some graph, hence admits a free action on some tree] does not contain any nontrivial finite subgroups.

\section{$\S 2 . \quad$ Commensurability Properties}

In this $\S$, we begin our study of the geometry of semi-graphs of profinite groups by considering various topics concerning commensurators and slimness.

In the following, we shall use the language of anabelioids of [Mzk4] — i.e., "multi-Galois categories" in the language of [SGA1] — which, in effect, amounts to working with profinite groups up to inner automorphism [cf., e.g., [Mzk4], Proposition 1.1.4]. If $\mathcal{X}$ is a connected anabelioid [i.e., a Galois category], then we shall denote the profinite fundamental group [for some choice of basepoint] of $\mathcal{X}$ by:

$$
\widehat{\pi}_{1}(\mathcal{X})
$$

As is well-known, this profinite group is, in a natural sense, independent of the choice of basepoint, up to inner automorphism. 
Definition 2.1. We shall refer to the following data $\mathcal{G}$ :

(a) a semi-graph $\mathbb{G}$;

(b) for each vertex $v$ of $\mathbb{G}$, a connected anabelioid $\mathcal{G}_{v}$;

(c) for each edge $e$ of $\mathbb{G}$, a connected anabelioid $\mathcal{G}_{e}$, together with, for each branch $b \in e$ abutting to a vertex $v$, a morphism of anabelioids $b_{*}: \mathcal{G}_{e} \rightarrow \mathcal{G}_{v}$

as a semi-graph of (connected) anabelioids [cf. the notion of a "semi-graph of profinite groups" introduced in [Mzk3], Appendix]. We shall refer to the various $\mathcal{G}_{v}, \mathcal{G}_{e}$ as the constituent anabelioids of $\mathcal{G}$. Given two semi-graphs of anabelioids, there is an evident notion of morphism between semi-graphs of anabelioids [cf. also Remark 2.4 .2 below]. If all of the $b_{*}$ 's are $\pi_{1}$-monomorphisms [i.e., induce injective homomorphisms on associated fundamental groups — cf. [Mzk4], Definition 1.1.12], then we shall say that $\mathcal{G}$ is of injective type. When the underlying semi-graph $\mathbb{G}$ is a graph, we shall refer to a semi-graph of anabelioids $\mathcal{G}$ as a graph of (connected) anabelioids.

Let $\mathcal{G}$ be a connected semi-graph of anabelioids [i.e., the underlying semigraph $\mathbb{G}$ is assumed to be connected]. If $\mathbb{G}$ has at least one vertex, then let us denote by

$$
\mathcal{B}(\mathcal{G})
$$

the category of objects given by data

$$
\left\{S_{v}, \phi_{e}\right\}
$$

where $v$ (respectively, $e$ ) ranges over the vertices (respectively, edges) of $\mathbb{G}$; for each vertex $v, S_{v} \in \operatorname{Ob}\left(\mathcal{G}_{v}\right)$; for each edge $e$, with branches $b_{1}, b_{2}$ abutting to vertices $v_{1}, v_{2}$, respectively, $\phi_{e}:\left\{\left(b_{1}\right)_{*}\right\}^{*} S_{v_{1}} \stackrel{\sim}{\rightarrow}\left\{\left(b_{2}\right)_{*}\right\}^{*} S_{v_{2}}$ is an isomorphism in $\mathcal{G}_{e}$, and morphisms given by morphisms [in the evident sense] between such data. If $\mathbb{G}$ has no vertices - and hence precisely one edge $e$, which is necessarily isolated - then we shall write $\mathcal{B}(\mathcal{G}) \stackrel{\text { def }}{=} \mathcal{G}_{e}$. One verifies immediately that this category $\mathcal{B}(\mathcal{G})$ is a connected anabelioid.

Now let $G^{\prime} \in \operatorname{Ob}(\mathcal{B}(\mathcal{G}))$; write

$$
\mathcal{B}^{\prime} \stackrel{\text { def }}{=} \mathcal{B}(\mathcal{G})_{G^{\prime}} \rightarrow \mathcal{B} \stackrel{\text { def }}{=} \mathcal{B}(\mathcal{G})
$$

for the corresponding finite étale covering of anabelioids [cf. [Mzk4], Definition 1.2 .2 , (i)]. Then it follows from the definition of the anabelioid $\mathcal{B}(\mathcal{G})$ associated to the semi-graph of anabelioids $\mathcal{G}$ [i.e., in terms of finite étale coverings of each 
of the constituent anabelioids of $\mathcal{G}$, together with gluing isomorphisms] that $\mathcal{B}^{\prime}$ itself arises naturally as the $\mathcal{B}(-)$ of some connected semi-graph of anabelioids $\mathcal{G}^{\prime}$ equipped with a morphism

$$
\mathcal{G}^{\prime} \rightarrow \mathcal{G}
$$

of semi-graphs of anabelioids which lies over some proper morphism of semigraphs:

$$
\mathbb{G}^{\prime} \rightarrow \mathbb{G}
$$

Here, if $v$ (respectively, $e$ ) is a vertex (respectively, edge) of $\mathbb{G}$, then the vertices $v^{\prime}$ (respectively, edges $e^{\prime}$ ) of $\mathbb{G}^{\prime}$ are the elements of the set of connected components of the [not necessarily connected!] anabelioid $\mathcal{B}^{\prime} \times_{\mathcal{B}} \mathcal{G}_{v}$ (respectively, $\mathcal{B}^{\prime} \times_{\mathcal{B}} \mathcal{G}_{e}$ ). The connected anabelioid $\mathcal{G}_{v^{\prime}}^{\prime}\left(\right.$ respectively, $\left.\mathcal{G}_{e^{\prime}}^{\prime}\right)$ is the connected component anabelioid of $\mathcal{B}^{\prime} \times_{\mathcal{B}} \mathcal{G}_{v}$ (respectively, $\mathcal{B}^{\prime} \times_{\mathcal{B}} \mathcal{G}_{e}$ ) determined by $v^{\prime}$ (respectively, $e^{\prime}$ ).

Let us denote by

$$
\Pi_{\mathbb{G}} \stackrel{\text { def }}{=} \widehat{\pi}_{1}(\mathcal{G}) \stackrel{\text { def }}{=} \widehat{\pi}_{1}(\mathcal{B}(\mathcal{G}))
$$

the fundamental group of the connected anabelioid $\mathcal{B}(\mathcal{G})$ relative to some basepoint. Put another way, if we choose basepoints for the constituent anabelioids of $\mathcal{G}$, then $\mathcal{G}$ determines a "semi-graph of profinite groups" [cf. [Mzk3], Appendix, except that here the underlying semi-graph is not necessarily finite], and one may think of $\Pi_{\mathbb{G}}$ as the profinite group associated to this semi-graph of profinite groups.

For each vertex $v$ (respectively, branch $b$ of an edge $e$ that abuts to the vertex $v$ ) of $\mathbb{G}$, let us write $\Pi_{v}$ (respectively, $\Pi_{b}$ ) for the fundamental group of the anabelioid $\mathcal{G}_{v}$ (respectively, $\mathcal{G}_{e}$ ) for some choice of basepoint. Thus, we have natural outer homomorphisms:

$$
\Pi_{v} \rightarrow \Pi_{\mathbb{G}} ; \quad \Pi_{b} \rightarrow \Pi_{\mathbb{G}}
$$

Moreover, the branch $b$ determines an associated outer homomorphism:

$$
\Pi_{b} \rightarrow \Pi_{v}
$$

If $\mathcal{G}$ is of injective type, then we shall also denote the image of $\Pi_{b}$ in $\Pi_{v}$, which is well-defined up to conjugation in $\Pi_{v}$, by $\Pi_{b}$. [Here, we note that the use of the subscript " $b$ " in the notation " $\Pi_{b}$ " - i.e., as opposed to " $e$ " in, for instance, the notation " $\mathcal{G}_{e}$ " - is useful in discussions concerning subgroups of $\Pi_{v}$ [as opposed to just subgroups of $\left.\Pi_{\mathbb{G}}\right]$.]

If $\mathbb{H} \subseteq \mathbb{G}$ is a [not necessarily connected] sub-semi-graph of $\mathbb{G}$, then we shall write $\mathcal{G}_{\mathbb{H}}$ for the semi-graph of anabelioids determined by restricting $\mathcal{G}$ to $\mathbb{H}$. 
That is to say, the underlying semi-graph of $\mathcal{G}_{\mathbb{H}}$ is $\mathbb{H}$, and for each component $c$ (i.e., either an edge or vertex) of $\mathbb{H}$, we let $\left(\mathcal{G}_{\mathbb{H}}\right)_{c} \stackrel{\text { def }}{=} \mathcal{G}_{c}$; if a branch $b$ of an edge $e$ of $\mathbb{H}$ abuts to a vertex $v$ of $\mathbb{H}$, then we take the associated morphism $b_{*}:\left(\mathcal{G}_{\mathbb{H}}\right)_{e} \rightarrow\left(\mathcal{G}_{\mathbb{H}}\right)_{v}$ to be the morphism associated to the corresponding branch of $\mathbb{G}$. If $\mathbb{H}$ is connected, then we shall write

\section{$\Pi_{\mathbb{H}}$}

for the fundamental group of $\mathcal{G}_{\mathbb{H}}$, for some choice of basepoint. Thus, we have a natural outer homomorphism $\Pi_{\mathbb{H}} \rightarrow \Pi_{\mathbb{G}}$.

Definition 2.2. $\quad$ (i) We shall refer to an arrow $\mathcal{G}^{\prime} \rightarrow \mathcal{G}$ that arises as in the above discussion from a finite étale covering of anabelioids $\mathcal{B}^{\prime} \stackrel{\text { def }}{=} \mathcal{B}(\mathcal{G})_{G^{\prime}} \rightarrow$ $\mathcal{B} \stackrel{\text { def }}{=} \mathcal{B}(\mathcal{G})$ as a finite étale covering $[$ of $\mathcal{G}]$.

(ii) If a morphism of semi-graphs of anabelioids satisfies the property that each of its induced morphisms between constituent anabelioids is an isomorphism (respectively, is finite étale; induces an homomorphism with open image between the respective $\widehat{\pi}_{1}(-)$ 's), then we shall say that the morphism is locally trivial (respectively, locally finite étale; locally open).

Remark 2.2.1. Note that by considering collections of normal open subgroups of $\Pi_{\mathbb{G}}$ whose intersection is trivial, one may think of $\Pi_{\mathbb{G}}$ as acting on a "universal pro-finite étale covering" of $\mathcal{G}$. In particular, $\Pi_{\mathbb{G}}$ acts naturally on the underlying "pro-semi-graph" of this "universal pro-finite étale covering". Moreover, it follows immediately from the definitions [cf. the discussion of the universal covering associated to a graph of groups in [Serre], Chapter I, p. 51] that the image of each $\Pi_{v}$ (respectively, $\Pi_{b}$ ) in $\Pi_{\mathbb{G}}$ is equal to the stabilizer of a compatible system of vertices (respectively, edges) of this pro-semi-graph.

Definition 2.3. $\quad$ Let $\mathcal{G}$ be a semi-graph of anabelioids of injective type, with underlying semi-graph $\mathbb{G}$.

(i) We shall say that $\mathcal{G}$ is of bounded order if there exists an integer $M \geq 1$ such that all of the $\widehat{\pi}_{1}\left(\mathcal{G}_{v}\right)$ 's, where $v$ ranges over the vertices of the underlying semi-graph $\mathbb{G}$, are finite groups of order dividing $M$.

(ii) We shall refer to a morphism of semi-graphs of anabelioids

$$
\mathcal{G} \rightarrow \mathcal{G}^{\prime}
$$


which induces an isomorphism on underlying semi-graphs $\mathbb{G} \stackrel{\sim}{\rightarrow} \mathbb{G}^{\prime}$ [relative to which we may identify $\left.\mathbb{G}, \mathbb{G}^{\prime}\right]$ and for which $\mathcal{G}^{\prime}$ is a semi-graph of anabelioids of bounded order as an approximator for $\mathcal{G}$. We shall say that an approximator is $\pi_{1}$-epimorphic if each of the induced morphisms between the respective constituent anabelioids is a $\pi_{1}$-epimorphism [i.e., induces a surjective homomorphism on associated fundamental groups — cf. [Mzk4], Definition 1.1.12].

(iii) We shall say that $\mathcal{G}$ is quasi-coherent if, for every integer $M \geq 1$, and every collection of finite étale coverings $\mathcal{H}_{c} \rightarrow \mathcal{G}_{c}$ of degree $\leq M$, where $c$ ranges over the components of $\mathbb{G}$, there exists an approximator

$$
\mathcal{G} \rightarrow \mathcal{G}^{\prime}
$$

such that, for each component $c$ of $\mathbb{G}$, the pull-back to $\mathcal{G}_{c}$ of the "universal covering" $\mathcal{H}_{c}^{\prime} \rightarrow \mathcal{G}_{c}^{\prime}$ of $\mathcal{G}_{c}^{\prime}$ [i.e., the finite étale covering determined by the trivial subgroup of $\widehat{\pi}_{1}\left(\mathcal{G}_{c}^{\prime}\right)$ ] splits $\mathcal{H}_{c} \rightarrow \mathcal{G}_{c}$. In this situation, we shall say that this approximator splits the given collection of coverings. We shall say that a quasi-coherent $\mathcal{G}$ is coherent if, for each component $c$ of $\mathbb{G}$, the profinite group $\widehat{\pi}_{1}\left(\mathcal{G}_{c}\right)$ is topologically finitely generated [which, as is well-known, implies that $\operatorname{Out}\left(\widehat{\pi}_{1}\left(\mathcal{G}_{c}\right)\right)$ is equipped with a natural profinite group structure].

Remark 2.3.1. Relative to the notation of Definition 2.3, (iii), by replacing the constituent anabelioids of $\mathcal{G}^{\prime}$ by the image anabelioids of the constituent anabelioids of $\mathcal{G}$ [cf. [Mzk4], Definition 1.1.7, (i)], one may always take the approximator of Definition 2.3, (iii), to be $\pi_{1}$-epimorphic.

Definition 2.4. Let $\mathcal{G}$ be a semi-graph of anabelioids of injective type, with underlying semi-graph $\mathbb{G}$.

(i) Let $v$ be a vertex of $\mathbb{G}$. If, for every integer $M \geq 1$, there exists a $\pi_{1}$-epimorphic approximator

$$
\mathcal{G} \rightarrow \mathcal{G}^{\prime}
$$

for $\mathcal{G}$ such that there exists a subgroup $N_{M} \subseteq \widehat{\pi}_{1}\left(\mathcal{G}_{v}^{\prime}\right)$ of order $\geq M$ which has trivial intersection with all of the conjugates, in $\widehat{\pi}_{1}\left(\mathcal{G}_{v}^{\prime}\right)$, of all of the $\widehat{\pi}_{1}\left(\mathcal{G}_{e}^{\prime}\right)$ [where $e$ ranges over the edges abutting to $v$ ], then we shall say that $v$ is elevated. If all of the vertices of $\mathbb{G}$ are elevated, then we shall say that $\mathcal{G}$ is totally elevated.

(ii) If, for every vertex $v$ of $\mathbb{G}$, the anabelioid $\mathcal{G}_{v}$ is slim, then we shall say that $\mathcal{G}$ is verticially slim. 
(iii) Let $e$ be a closed edge of $\mathbb{G}$. If there exists a finite étale covering $\mathcal{G}^{\prime} \rightarrow \mathcal{G}$ of $\mathcal{G}$ such that the underlying graph $\mathbb{G}^{\prime}$ of $\mathcal{G}^{\prime}$ contains a pair of distinct coverticial edges $e_{a}, e_{b}$, both of which map to $e$ in $\mathbb{G}$, then we shall say that $e$ is sub-coverticial. If, for every finite étale covering $\mathcal{G}^{\prime \prime} \rightarrow \mathcal{G}$ of $\mathcal{G}$, it holds that every edge $e^{\prime \prime}$ of the underlying graph $\mathbb{G}^{\prime \prime}$ of $\mathcal{G}^{\prime \prime}$ that maps to $e$ is sub-coverticial, then we shall say that $e$ is universally sub-coverticial. If every closed edge of $\mathbb{G}$ is sub-coverticial (respectively, universally sub-coverticial), then we shall say that $\mathcal{G}$ is totally sub-coverticial (respectively, totally universally sub-coverticial).

(iv) Let $e$ be an edge of $\mathbb{G}$. We shall say that $e$ is aloof (respectively, estranged) if, for every vertex $v$ to which some branch $b$ of $e$ abuts and every $g \in \Pi_{v}$, the intersection in $\Pi_{v}$ of $\Pi_{b}$ with any subgroup of the form $g \cdot \Pi_{b^{\prime}} \cdot g^{-1}$, where either $b^{\prime} \neq b$ is a branch of an edge that abuts to $v$ or $b^{\prime}=b$ and $g \notin \Pi_{b}$, has infinite index in $\Pi_{b}$ (respectively, and is, in fact, trivial). If every edge of $\mathbb{G}$ is aloof (respectively, estranged), then we shall say that $\mathcal{G}$ is totally aloof (totally estranged).

Remark 2.4.1. It is immediate that "(totally) estranged" implies "(totally) aloof". Moreover, one verifies easily that if $\mathcal{G}^{\prime} \rightarrow \mathcal{G}$ is a finite étale covering, and $v^{\prime}$ (respectively, $e^{\prime} ; e^{\prime} ; e^{\prime}$ ) is a(n) vertex (respectively, edge; edge; edge) of $\mathbb{G}^{\prime}$ that maps to a(n) elevated vertex $v$ (respectively, universally subcoverticial edge $e$; aloof edge $e$; estranged edge $e$ ) of $\mathbb{G}$, then $v^{\prime}$ (respectively, $e^{\prime} ; e^{\prime} ; e^{\prime}$ ) is itself elevated (respectively, universally sub-coverticial; aloof; estranged).

Remark 2.4.2. Let $\phi: \mathcal{G} \rightarrow \mathcal{H}$ be a morphism between semi-graphs of anabelioids of injective type. Concretely speaking, this means that we are given, for each vertex $v$ (respectively, edge e) of $\mathcal{G}$ mapping to a vertex $w$ (respectively, edge $f$ ) of $\mathcal{H}$, a 1-morphism of anabelioids

$$
\phi_{v}: \mathcal{G}_{v} \rightarrow \mathcal{H}_{w} \text { (respectively, } \phi_{e}: \mathcal{G}_{e} \rightarrow \mathcal{H}_{f} \text { ) }
$$

together with an isomorphism $\phi_{b}$ of the composite 1-morphism of anabelioids $\mathcal{G}_{e} \rightarrow \mathcal{G}_{v} \rightarrow \mathcal{H}_{w}$ with the composite 1-morphism of anabelioids $\mathcal{G}_{e} \rightarrow \mathcal{H}_{f} \rightarrow \mathcal{H}_{w}$ whenever a branch $b$ of $e$ abuts $v$. That is to say, strictly speaking, $\phi$ is a "1-morphism"; the 1-morphisms from $\mathcal{G}$ to $\mathcal{H}$ form a category, of which $\phi$ is an object; one can then speak of isomorphisms between various objects of this category.

On the other hand, observe that if we restrict our attention to locally open morphisms $\phi$ between totally aloof semi-graphs of anabelioids, then it follows formally from the definitions [cf. also [Mzk4], Corollary 1.1.6] that: 
The isomorphism class of $\phi$ is completely determined by the isomorphism class of the $\phi_{v}$.

If, moreover, we restrict our attention to locally open morphisms $\phi$ between totally aloof, verticially slim semi-graphs of anabelioids, then:

The 1-morphism $\phi$ has no nontrivial automorphisms.

That is to say, as long as we restrict our attention to locally open morphisms [e.g., locally finite étale] between totally aloof, verticially slim semi-graphs of anabelioids, we may work with such morphisms as if they are simply "morphisms in a category", rather than 1-morphisms in a 2-category [cf. the situation for finite étale morphisms between slim anabelioids: [Mzk4], Proposition 1.2.5]. In the following, we shall often take this point of view without further mention.

Proposition 2.5 (Injectivity). Let $\mathcal{G}$ be a connected, quasicoherent graph of anabelioids. Let $\mathbb{H} \subseteq \mathbb{G}$ be a connected subgraph of the underlying graph $\mathbb{G}$ of $\mathcal{G}$. Then:

(i) The natural morphisms $\Pi_{b} \rightarrow \Pi_{\mathbb{G}}, \Pi_{v} \rightarrow \Pi_{\mathbb{G}}, \Pi_{\mathbb{H}} \rightarrow \Pi_{\mathbb{G}}$ are injective. By abuse of notation, we will denote their images, which are well-defined up to conjugation in $\Pi_{\mathbb{G}}$, by $\Pi_{b}, \Pi_{v}, \Pi_{\mathbb{H}}$, respectively.

(ii) Suppose that $\mathcal{G}$ is of bounded order. Then there exists a normal open subgroup $H \subseteq \Pi_{\mathbb{G}}$, such that all of the natural morphisms $\Pi_{b} \rightarrow \Pi_{\mathbb{G}} / H$, $\Pi_{v} \rightarrow \Pi_{\mathbb{G}} / H$ are injective.

Proof. First, we consider assertion (i). Since the natural morphism $\Pi_{b} \rightarrow \Pi_{v}$ is injective, and the morphism $\Pi_{v} \rightarrow \Pi_{\mathbb{G}}$ may be considered as a special case of the morphism $\Pi_{\mathbb{H}} \rightarrow \Pi_{\mathbb{G}}$ [i.e., the case where $\mathbb{H}$ consists of a single vertex and no edges], it suffices to show that any finite étale Galois covering of $\mathcal{G}_{\mathbb{H}}$ may be split by a finite étale covering pulled back from $\mathcal{G}$. Note that this is immediate in the locally trivial case. Indeed, in this case, it suffices to extend the given finite étale covering from $\mathcal{G}_{\mathbb{H}}$ to the remainder of $\mathcal{G}$ by gluing [which is always possible, by the local triviality assumption!].

Thus, by our assumption of quasi-coherence, it suffices to construct, under the further assumption that $\mathcal{G}$ is of bounded order, a finite étale covering of $\mathcal{G}$ each of whose constituent anabelioids is trivial. We construct such a covering by gluing: Let $M \geq 1$ be an integer such all of the orders $\left[\widehat{\pi}_{1}\left(\mathcal{G}_{v}\right): 1\right]$ divide $M$. Over the vertex $v$, we take the covering to be the union of $M /\left[\widehat{\pi}_{1}\left(\mathcal{G}_{v}\right): 1\right]$ copies of some "universal covering" of $\mathcal{G}_{v}$ [i.e., the 
finite étale covering determined by the trivial subgroup of $\left.\widehat{\pi}_{1}\left(\mathcal{G}_{v}\right)\right]$. If $e$ is an edge that abuts to the vertex $v$, then the restriction of this covering to $e$ is a union of $M /\left[\widehat{\pi}_{1}\left(\mathcal{G}_{e}\right): 1\right]$ copies of some universal covering of $\mathcal{G}_{e}$ - i.e., a covering of $\mathcal{G}_{e}$ whose isomorphism class is independent of $v$ ! Thus, by choosing appropriate gluing isomorphisms, we obtain a covering of $\mathcal{G}$ having the desired properties.

As for assertion (ii), the Galois closure of a covering such as that constructed in the preceding paragraph determines a normal open subgroup $H \subseteq$ $\Pi_{\mathbb{G}}$ having the properties asserted in assertion (ii).

Remark 2.5.1. Note that the quasi-coherence hypothesis in the injectivity assertion of Proposition 2.5, (i), is by no means superfluous: For instance, let $G \stackrel{\text { def }}{=} \mathbb{Z} / l \mathbb{Z}$ [where $l$ is a prime number]; write $G^{\mathbb{N}}$ for the profinite group given by taking the direct product of a collection of copies of $G$ indexed by the set of natural numbers $\mathbb{N}$. Write $\alpha: G^{\mathbb{N}} \hookrightarrow G^{\mathbb{N}}$ for the continuous injection induced by the injection $\mathbb{N} \hookrightarrow \mathbb{N}$ given by $\mathbb{N} \ni n \mapsto n+1$. Then let us observe that there does not exist a proper open subgroup $H \subseteq G^{\mathbb{N}}$ such that $H=\alpha^{-1}(H)$. In particular, if one takes $\mathbb{G}$ to be the graph with precisely one vertex and one edge [both of whose branches abut to the unique vertex], and $\mathcal{G}$ to be the graph of anabelioids with underlying graph $\mathbb{G}$ such that the unique vertex and unique edge are equipped with the anabelioid $\mathcal{B}\left(G^{\mathbb{N}}\right)$ and the morphisms $\mathcal{B}\left(G^{\mathbb{N}}\right) \rightarrow \mathcal{B}\left(G^{\mathbb{N}}\right)$ corresponding to the two branches of the unique edge are given by the identity and $\mathcal{B}(\alpha)$, then one concludes from the above observation that the profinite fundamental group associated to this graph of anabelioids $\mathcal{G}$ is trivial.

Proposition 2.6 (Commensurability). Let $\mathcal{G}$ be a connected, quasicoherent graph of anabelioids. Let $\mathbb{H}, \mathbb{K} \subseteq \mathbb{G}$ be connected subgraphs of the underlying graph $\mathbb{G}$ of $\mathcal{G}$. Suppose that there exists a component $c$ of $\mathbb{H}$ that does not belong to $\mathbb{K}$ and which is either an elevated vertex or a subcoverticial edge $[$ i.e., relative to $\mathcal{G}]$. Then the intersection of $\Pi_{\mathbb{H}}$ with any conjugate of $\Pi_{\mathbb{K}}$ has infinite index in $\Pi_{\mathbb{H}}$. In particular, no conjugate of $\Pi_{\mathbb{H}}$ is commensurable to a conjugate of $\Pi_{\mathbb{K}}$.

Proof. First, we consider the case where $c=v$ is a vertex. We may assume without loss of generality that $\Pi_{\mathbb{K}} \bigcap \Pi_{\mathbb{H}}$ has finite index in $\Pi_{\mathbb{H}}$. Then by taking " $M$ " in Definition 2.4 , (i), to be

$$
\left[\Pi_{\mathbb{H}}: \Pi_{\mathbb{K}} \bigcap \Pi_{\mathbb{H}}\right]+1
$$


and applying the fact that $\mathcal{G}$ is elevated at $v$, we obtain the existence of a $\pi_{1}$-epimorphic approximator

$$
\mathcal{G} \rightarrow \mathcal{G}^{\prime}
$$

such that, if we denote the various fundamental groups associated to $\mathcal{G}^{\prime}$ by means of a "dash", then there is a subgroup $N_{M} \subseteq \Pi_{v}^{\prime}$ of order $\geq M$ that has trivial intersection with each of the conjugates of the $\Pi_{b}^{\prime}$, for branches $b$ that abut to $v$. Since $\mathcal{G}^{\prime}$ is of bounded order, it follows from Proposition 2.5, (ii), that there exists a normal open subgroup $K \subseteq \Pi_{\mathbb{K}}^{\prime}$ such that $\Pi_{w}^{\prime}$ injects into $\Pi_{\mathbb{K}}^{\prime} / K$, for all vertices $w$ of $\mathbb{K}$; moreover, it follows that there exists an integer $M^{\prime}$ that is divisible both by $\left[\Pi_{\mathbb{K}}^{\prime}: K\right]$ and by twice the orders of all of the $\Pi_{w}^{\prime}$, as $w$ ranges over the vertices of $\mathbb{G}$.

Now we construct a [not necessarily connected!] finite étale covering

$$
\mathcal{G}^{\prime \prime} \rightarrow \mathcal{G}^{\prime}
$$

as follows: Over $\mathbb{K}$, we take this covering to be the union of $M^{\prime} /\left[\Pi_{\mathbb{K}}^{\prime}: K\right]$ copies of the covering defined by the normal open subgroup $K \subseteq \Pi_{\mathbb{K}}^{\prime}$. Over vertices $w \neq v$ not contained in $\mathbb{K}$, we take this covering to be a union of $M^{\prime} /\left[\Pi_{w}^{\prime}: 1\right]$ copies of a "universal covering" of $\mathcal{G}_{w}^{\prime}$. Over $v$, we take this covering to be a union of $M^{\prime} / 2\left[\Pi_{v}^{\prime}: N_{M}\right]$ copies of the covering defined by the $\Pi_{v}^{\prime}$-set $\Pi_{v}^{\prime} / N_{M}$ and of $M^{\prime} / 2\left[\Pi_{v}^{\prime}: 1\right]$ copies of a "universal covering" of $\mathcal{G}_{v}^{\prime}$. Note that the restriction of any of these coverings over $w$ or $v$ to an abutting edge $e$ is isomorphic to a union of "universal coverings" of $\mathcal{G}_{e}^{\prime}$. Thus, by choosing appropriate gluing isomorphisms, we obtain a covering $\mathcal{G}^{\prime \prime} \rightarrow \mathcal{G}^{\prime}$.

On the other hand, the existence of this covering leads to a contradiction, as follows: This covering determines a finite $\Pi_{\mathbb{G}}$-set $S$; write $H^{\prime \prime} \stackrel{\text { def }}{=} \operatorname{Ker}\left(\Pi_{\mathbb{G}} \rightarrow\right.$ $\operatorname{Aut}(S)$ ); denote the images in $\Pi_{\mathbb{G}} / H^{\prime \prime}$ of the various fundamental groups under consideration by means of a "double dash". Thus, we have equalities $\Pi_{\mathbb{K}}^{\prime \prime}=\Pi_{\mathbb{K}} / K, \Pi_{v}^{\prime \prime}=\Pi_{v}^{\prime}$ [because of the existence of "universal coverings" in the restriction of $\mathcal{G}^{\prime \prime}$ to $v$ ] and inequalities as follows:

$$
\left[\Pi_{\mathbb{H}}^{\prime \prime}: \Pi_{\mathbb{K}}^{\prime \prime} \bigcap \Pi_{\mathbb{H}}^{\prime \prime}\right] \leq\left[\Pi_{\mathbb{H}}: \Pi_{\mathbb{K}} \bigcap \Pi_{\mathbb{H}}\right]<M
$$

Moreover, since $\Pi_{\mathbb{K}}^{\prime \prime}$ acts freely on $S$, it follows that $\Pi_{\mathbb{K}}^{\prime \prime} \bigcap \Pi_{\mathbb{H}}^{\prime \prime}$ also acts freely on $S$, and hence that the isotropy subgroup $I \subseteq \Pi_{\mathbb{H}}^{\prime \prime}$ associated to an element of $S$ has order $\leq\left[\Pi_{\mathbb{H}}^{\prime \prime}: \Pi_{\mathbb{K}}^{\prime \prime} \cap \Pi_{\mathbb{H}}^{\prime \prime}\right]<M$. In particular, $I_{v} \stackrel{\text { def }}{=} I \bigcap \Pi_{v}^{\prime \prime}$ has order $<M$. On the other hand, by the construction of $\mathcal{G}^{\prime \prime}$, it follows that for some such isotropy group $I_{v} \subseteq \Pi_{v}^{\prime \prime}=\Pi_{v}^{\prime}$, we have $N_{M} \subseteq I_{v}$, where we recall that $N_{M}$ has order $\geq M$, a contradiction. 
Next, we consider the case where $c=e$ is an edge. Since $e$ is sub-coverticial, it suffices [by replacing $\mathcal{G}$ by a finite étale covering of $\mathcal{G}$ ] to show that $\Pi_{\mathbb{K}} \bigcap \Pi_{\mathbb{H}}$ has infinite index in $\Pi_{\mathbb{H}}$ under the assumption that $\mathbb{H}$ contains a pair of distinct coverticial edges $e_{a}, e_{b}$, neither of which is contained in $\mathbb{K}$. Thus, let us suppose that $\Pi_{\mathbb{K}} \bigcap \Pi_{\mathbb{H}}$ has finite index in $\Pi_{\mathbb{H}}$; set $M \stackrel{\text { def }}{=}\left[\Pi_{\mathbb{H}}: \Pi_{\mathbb{K}} \bigcap \Pi_{\mathbb{H}}\right]+1$.

Now observe that by using the loop $\mathbb{L}$ of $\mathbb{H}$ constituted by $e_{a}, e_{b}$, we may construct a finite graph-covering of degree $M$

$$
\mathbb{G}^{\prime} \rightarrow \mathbb{G}
$$

which is trivial over $\mathbb{K}$, but connected over $\mathbb{L}$. Then considering the actions of $\Pi_{\mathbb{H}}, \Pi_{\mathbb{K}}$ on the corresponding finite $\Pi_{\mathbb{G}^{\mathbb{S}}}$-set yields a contradiction. This completes the proof.

Remark 2.6.1. Note that the hypothesis in Proposition 2.6 concerning the existence of an elevated vertex or a sub-coverticial edge is by no means superfluous: Indeed, let $G$ be a profinite group, $H \subseteq G$ an open subgroup. Suppose that $\mathbb{G}$ is the graph with precisely three vertices, labeled $1,2,3$, and precisely two edges, one of which joins the vertices 1,2 , the other of which joins the vertices 2, 3. Suppose that the graph of anabelioids structure of $\mathcal{G}$ is given by equipping the vertex 1 (respectively, $2 ; 3$ ) with the anabelioid $\mathcal{B}(G)$ (respectively, $\mathcal{B}(H) ; \mathcal{B}(H)$ ), the two edges with the anabelioid $\mathcal{B}(H)$; we take the morphisms $\mathcal{B}(H) \rightarrow \mathcal{B}(H), \mathcal{B}(H) \rightarrow \mathcal{B}(G)$ corresponding to the various branches of edges to be the morphisms induced by the identity and inclusion homomorphisms. Then one verifies immediately that the profinite fundamental group associated to this graph of anabelioids is naturally isomorphic to $G$, but that if one takes $\mathbb{H}$ to be the subgraph determined by the [non-elevated!] vertex 1 and $\mathbb{K}$ to be the subgraph determined by the vertices 2,3 and the $[$ non-subcoverticial!] edge that joins them, then the conclusion of Proposition 2.6 is false [even if one interchanges $\mathbb{H}, \mathbb{K}$ ].

Corollary 2.7 (Slimness and Commensurators). Let $\mathcal{G}$ be a connected, quasi-coherent graph of anabelioids. Let $\mathbb{H}, \mathbb{K} \subseteq \mathbb{G}$ be connected subgraphs of the underlying graph $\mathbb{G}$ of $\mathcal{G}$. Suppose that every vertex of $\mathbb{H}, \mathbb{K}$ is elevated [i.e., relative to $\mathcal{G}]$. Then:

(i) We have $C_{\Pi_{\mathbb{G}}}\left(\Pi_{\mathbb{H}}\right)=\Pi_{\mathbb{H}}$. In particular, if $v$ is an elevated vertex of $\mathbb{G}$, then $C_{\Pi_{\mathbb{G}}}\left(\Pi_{v}\right)=\Pi_{v}$.

(ii) Suppose that $\mathbb{H}$ contains a vertex $v$ such that $\mathcal{G}_{v}$ is slim. Then the natural morphism $\mathcal{B}\left(\mathcal{G}_{\mathbb{H}}\right) \rightarrow \mathcal{B}(\mathcal{G})$ is relatively slim [cf. [Mzk4], Definition 1.2.9]. In particular, $\mathcal{B}(\mathcal{G})$ is slim. 
(iii) Suppose that every edge of $\mathbb{H}, \mathbb{K}$ is sub-coverticial [i.e., relative to $\mathcal{G}]$ and that $\Pi_{\mathbb{H}}$ is commensurable to a conjugate of $\Pi_{\mathbb{K}}$ in $\Pi_{\mathbb{G}}$. Then $\mathbb{H}=\mathbb{K}$.

Proof. $\quad$ First, we consider assertion (i). Suppose that $g \in C_{\Pi_{\mathbb{G}}}\left(\Pi_{\mathbb{H}}\right)$, but $g \notin \Pi_{\mathbb{H}}$. Then there exists a connected finite Galois étale covering

$$
\mathcal{G}^{\prime} \rightarrow \mathcal{G}
$$

- whose restriction to $\mathcal{H} \stackrel{\text { def }}{=} \mathcal{G}_{\mathbb{H}}$, we denote by $\mathcal{H}^{\prime} \rightarrow \mathcal{H}$ - satisfying the property that there exists a connected component $\mathbb{H}^{\prime \prime}$ of $\mathbb{H}^{\prime}$ such that $g \cdot \mathbb{H}^{\prime \prime} \neq \mathbb{H}^{\prime \prime}$ in $\mathbb{G}^{\prime}$. Since [as one verifies immediately] $\mathbb{H}^{\prime}$ injects into $\mathbb{G}^{\prime}$ as a subgraph, it thus follows that $g \cdot \mathbb{H}^{\prime \prime} \cap \mathbb{H}^{\prime \prime}=\emptyset\left[\right.$ in $\left.\mathbb{G}^{\prime}\right]$. Thus, assertion (i) follows from Proposition 2.6.

Next, we consider assertion (ii). By assertion (i), we have, for any open subgroup $H \subseteq \Pi_{\mathbb{H}}$ :

$$
Z_{\Pi_{\mathbb{G}}}(H) \subseteq Z_{\Pi_{\mathbb{G}}}\left(H \bigcap \Pi_{v}\right) \subseteq C_{\Pi_{\mathbb{G}}}\left(\Pi_{v}\right)=\Pi_{v}
$$

Thus, we conclude [since $\mathcal{G}_{v}$ is slim] that $Z_{\Pi_{\mathbb{G}}}(H) \subseteq Z_{\Pi_{v}}\left(H \bigcap \Pi_{v}\right)=\{1\}$.

Finally, assertion (iii) is a formal consequence of Proposition 2.6.

Remark 2.7.1. There is a certain overlap between the content of Corollary 2.7 and the results obtained in [HR]. The techniques of [HR], however, are more group-theoretic in nature and somewhat different in spirit from those employed in the present exposition.

Remark 2.7.2. $\quad$ Note that Corollary 2.7, (i), implies in particular that [in the notation of loc. cit.] if $J \subseteq \Pi_{\mathbb{G}}$ is any [not necessarily closed!] subgroup such that $\Pi_{v} \subseteq J$, then we have

$$
C_{J}\left(\Pi_{v}\right) \subseteq C_{\Pi_{\mathbb{G}}}\left(\Pi_{v}\right)=\Pi_{v}
$$

- i.e., $\Pi_{v}$ is commensurably terminal in $J$.

Next, we consider some concrete examples:

Example 2.8. Trivial Edge Anabelioids. One verifies immediately that every semi-graph of anabelioids $\mathcal{G}$ such that $\mathcal{G}_{e}$ is trivial for all edges $e$ is quasi-coherent. In this case, a vertex $v$ of $\mathcal{G}$ is elevated if and only if $\Pi_{v}$ is infinite. Similarly, a closed edge abutting to vertices $v, w$ is sub-coverticial 
(respectively, universally sub-coverticial) if and only if both $\Pi_{v}$ and $\Pi_{w}$ are nontrivial (respectively, infinite).

Definition 2.9. $\quad$ Let $\Sigma$ be a set of prime numbers.

(i) We shall refer to as a $\Sigma$-integer any positive integer each of whose prime factors belongs to $\Sigma$.

(ii) Let $\mathcal{A}$ be a connected anabelioid. Then we shall refer to as the pro- $\Sigma$ completion of $\mathcal{A}$ the connected anabelioid constituted by the full subcategory of $\mathcal{A}$ determined by the objects dominated by a Galois covering of the final object of $\mathcal{A}$ whose degree is a $\Sigma$-integer. Similarly, given a semi-graph of anabelioids, we shall refer to as the pro- $\Sigma$ completion of the given semi-graph of anabelioids the semi-graph of anabelioids obtained by replacing each constituent anabelioid by its pro- $\Sigma$ completion.

Example 2.10. Stable Curves. Let $\Sigma$ be a nonempty set of prime numbers. Suppose that $\mathcal{G}$ is a semi-graph of anabelioids with the property that each $\Pi_{v}$ is the maximal pro- $\Sigma$ quotient of the fundamental group of a hyperbolic Riemann surface of finite type, and that each $\Pi_{b} \rightarrow \Pi_{v}$ is the inclusion morphism of the inertia group of one of the cusps. Now observe that a hyperbolic Riemann surface of finite type admits Galois coverings of degree a $\Sigma$-integer which are ramified with ramification index equal to an arbitrary given $\Sigma$-integer at a given cusp, but which are unramified at all other cusps. In light of this observation [together with various other properties that follow immediately from the well-known structure of fundamental groups of hyperbolic Riemann surfaces of finite type], one verifies immediately that $\mathcal{G}$ is coherent, totally elevated, totally universally sub-coverticial, totally estranged [cf. the proof of [Mzk3], Lemma 1.3.7], and verticially slim [cf. the proof of [Mzk3], Lemma 1.3.1]. In particular, the pro- $\Sigma$ completion of the semi-graph of anabelioids determined by the semi-graph of profinite groups with compact structure associated to a pointed stable curve over an algebraically closed field of characteristic 0 [cf. [Mzk3], Appendix] satisfies these properties. Similarly, if $\Sigma$ contains at least one prime $\neq p$, then a hyperbolic curve over a separably closed field of characteristic $p$ admits Galois coverings of degree a $\Sigma$-integer which are ramified with ramification index equal to an arbitrary given $\Sigma$-integer prime to $p$ at a given cusp, but which are unramified at all other cusps; in light of this observation [together with various other well-known properties of fundamental groups of hyperbolic curves over separably closed field of characteristic $p$ ], one verifies easily that the pro- $\Sigma$ completion of the semi-graph of anabelioids determined 
by the semi-graph of profinite groups with compact structure associated to a stable curve over a separably closed field of characteristic $p>0$ is coherent, totally elevated, totally universally sub-coverticial, totally estranged [cf. the proof of [Mzk3], Lemma 1.3.12], and verticially slim [cf. the proof of [Mzk3], Lemma 1.3.10].

Remark 2.10.1. In the case of Example 2.10, it is not difficult to show, using exactly the same techniques as those used in the proofs of Proposition 2.6, Corollary 2.7, that $C_{\Pi_{\mathbb{G}}}\left(\Pi_{b}\right)=\Pi_{b}$. Since, however, we shall not need this result in the following, and, moreover, a precise description of the condition on edges in the case of a more general $\mathcal{G}$ necessary to carry out such an argument [i.e., the analogue for edges of the notion of an "elevated vertex"] would be rather technical to write out in detail, we leave the task of working out the routine details to the interested reader.

The case of Example 2.10 [cf. also Example 3.10 below] motivates the following extension of the notion of a "morphism of semi-graphs of anabelioids": First, we observe that any semi-graph $\mathbb{G}$ may be regarded as a category

$$
\operatorname{Cat}(\mathbb{G})
$$

as follows: The objects of this category are the components [i.e., vertices and edges] of $\mathbb{G}$. The morphisms of this category are the identity morphisms of the components and the branches of edges [i.e., if $b$ is a branch of an edge $e$ that abuts to a vertex $v$, then we regard $b$ as a morphism $e \rightarrow v$ ] of $\mathbb{G}$. Thus, if $\mathbb{G}$ is the underlying semi-graph of a semi-graph of anabelioids $\mathcal{G}$, then for every object $c$ of Cat $(\mathbb{G})$, we have an anabelioid $\mathcal{G}_{c}$, and for every morphism $b: e \rightarrow v$ of Cat $(\mathbb{G})$, we have a morphism of anabelioids $b_{*}: \mathcal{G}_{e} \rightarrow \mathcal{G}_{v}$.

Definition 2.11. Let $\mathcal{G}, \mathcal{H}$ be semi-graphs of anabelioids. Then a generalized morphism of semi-graphs of anabelioids

$$
\Phi: \mathcal{G} \rightarrow \mathcal{H}
$$

is defined to be a collection of data, as follows:

(a) a functor $\operatorname{Cat}(\Phi): \operatorname{Cat}(\mathbb{G}) \rightarrow \operatorname{Cat}(\mathbb{H})$;

(b) for every object $c$ of $\operatorname{Cat}(\mathbb{G})$ that is mapped by $\operatorname{Cat}(\Phi)$ to an object $d$ of $\operatorname{Cat}(\mathbb{H})$, a morphism of anabelioids $\Phi_{c}: \mathcal{G}_{c} \rightarrow \mathcal{H}_{d}$; 
(c) for every arrow $\phi: c \rightarrow c^{\prime}$ of $\operatorname{Cat}(\mathbb{G})$ that is mapped by Cat $(\Phi)$ to an arrow $\psi: d \rightarrow d^{\prime}$ of $\operatorname{Cat}(\mathbb{H})$, an isomorphism $\Phi_{\phi}: \psi_{*} \circ \Phi_{c} \stackrel{\sim}{\rightarrow} \Phi_{c^{\prime}} \circ \phi_{*}$, such that $\Phi_{\phi}$ is the identity whenever $\phi$ is an identity morphism.

Remark 2.11.1. It is immediate from the definitions that every [nongeneralized] morphism of semi-graphs of anabelioids determines a generalized morphism of semi-graphs of anabelioids. Also, just as in the non-generalized case, it is immediate from the definitions that every generalized morphism of semi-graphs of connected anabelioids

$$
\Phi: \mathcal{G} \rightarrow \mathcal{H}
$$

determines, in a natural fashion, a morphism $\mathcal{B}(\mathcal{G}) \rightarrow \mathcal{B}(\mathcal{H})$ between the associated anabelioids.

\section{$\S 3 . \quad$ The Tempered Fundamental Group}

In this $\S$, we define and study the basic properties of the tempered fundamental group of a semi-graph of anabelioids. The notion of the tempered fundamental group is introduced in [André], §4. In the present manuscript, however, we wish to study this notion from a more categorical point of view.

Let $\Pi$ be a topological group. Then let us write

$$
\mathcal{B}^{\text {temp }}(\Pi)
$$

for the category whose objects are countable [i.e., of cardinality $\leq$ the cardinality of the set of natural numbers], discrete sets equipped with a continuous $\Pi$-action and whose morphisms are morphisms of П-sets.

Definition 3.1. $\quad$ (i) If $\Pi$ may be written as an inverse limit of an inverse system of surjections of countable discrete topological groups, then we shall say that $\Pi$ is tempered.

(ii) Any category equivalent to a category of the form $\mathcal{B}^{\text {temp }}(\Pi)$ for some tempered topological group $\Pi$ will be referred to as a connected temperoid. Any category equivalent to a product [in the sense of a product of categories] of a countable [hence possibly empty!] collection of connected temperoids will be referred to as a temperoid.

(iii) Let $\mathcal{T}_{1}, \mathcal{T}_{2}$ be temperoids. Then a morphism $\phi: \mathcal{T}_{1} \rightarrow \mathcal{T}_{2}$ is defined to be a functor $\phi^{*}: \mathcal{T}_{2} \rightarrow \mathcal{T}_{1}$ that preserves finite limits and countable colimits. A morphism $\phi$ will be called rigid if the functor $\phi^{*}$ is rigid [cf. $§ 0$ ]. 
(iv) A connected object $T$ of a temperoid $\mathcal{T}$ will be called Galois if, for any two arrows $\psi_{1}, \psi_{2}: S \rightarrow T$ of $\mathcal{T}$, where $S$ is connected, there exists a [unique] automorphism $\alpha \in \operatorname{Aut}_{\mathcal{T}}(T)$ of $T$ such that $\psi_{1}=\alpha \circ \psi_{2}$.

Remark 3.1.1. Observe that every profinite group is tempered. Moreover, just as in the case of profinite groups, if a tempered group may be written as an inverse limit of an inverse system indexed by a countable set of surjections of countable discrete topological groups, then the group is countably generated [i.e., generated as a topological group by a countable set of generators].

Remark 3.1.2. $\quad$ Suppose that $\Pi$ is tempered. Then every open subgroup of $\Pi$ is closed and of countable index in $\Pi$. Moreover, the topology of $\Pi$ admits a basis of open normal subgroups. If $H \subseteq \Pi$ is an arbitrary subgroup, then the $\Pi$-set $\Pi / H$ forms an object of $\mathcal{B}^{\text {temp }}(\Pi)$ if and only if $H$ is open. If $H_{1}, H_{2} \subseteq \Pi$ are open, then there is a natural bijection between the morphisms $\Pi / H_{1} \rightarrow \Pi / H_{2}$ and the cosets $h \cdot H_{2}$ satisfying $h^{-1} \cdot H_{1} \cdot h \subseteq H_{2}$. In particular, if $T_{1}, T_{2}$ are objects of a temperoid $\mathcal{T}$, and $T_{1}$ is connected, then the set $\operatorname{Hom}_{\mathcal{T}}\left(T_{1}, T_{2}\right)$ is countable. If $\Pi^{\prime}$ is also tempered, then any continuous homomorphism $\Pi \rightarrow \Pi^{\prime}$ determines [by composing the action of $\Pi^{\prime}$ on a $\Pi^{\prime}$ set with this homomorphism so as to obtain a П-set] a morphism of connected temperoids $\mathcal{B}^{\text {temp }}(\Pi) \rightarrow \mathcal{B}^{\text {temp }}\left(\Pi^{\prime}\right)$.

Remark 3.1.3. $\quad$ Suppose that $\Pi$ is tempered. Then an object of $\mathcal{B}^{\text {temp }}(\Pi)$ is Galois if and only if it is isomorphic to the object determined by a $\Pi$-set of the form $\Pi / N$, where $N \subseteq \Pi$ is an open normal subgroup. Alternatively, a connected object $T$ of $\mathcal{T} \stackrel{\text { def }}{=} \mathcal{B}^{\text {temp }}(\Pi)$ is Galois if and only if the product $T \times T$ is isomorphic to the coproduct of copies of $T$ indexed by the elements of the [countable!] set $\operatorname{Aut}_{\mathcal{T}}(T)$, where the restriction to the copy labeled by $\sigma \in \operatorname{Aut}_{\mathcal{T}}(T)$ of the projection to the first (respectively, second) factor of $T \times T$ is given by the identity (respectively, $\sigma$ ).

Remark 3.1.4. Note that if $\mathcal{X}$ is an anabelioid (respectively, connected anabelioid), then

$$
\mathcal{X}^{\top \stackrel{\text { def }}{=}}\left(\mathcal{X}^{0}\right)^{\top}
$$

[cf. §0] is a temperoid (respectively, connected temperoid). We shall refer to $\mathcal{X}^{\top}$ as the temperification of $\mathcal{X}$. Just as in the case of anabelioids, the decomposition of a temperoid into a countable coproduct of connected temperoids each of which we shall refer to as a connected component of the original temperoid - may be recovered completely category-theoretically from the categorical 
structure of the original temperoid [i.e., by considering decompositions of the terminal object — cf. [Mzk4], Definitions 1.1.8, 1.1.9]. Also, just as in the case of anabelioids, to give a morphism between two temperoids is equivalent to giving, for each connected component of the domain temperoid, a morphism [of connected temperoids] — which we shall refer to as a component morphism of the morphism - from that connected component to some connected component of the codomain temperoid. Finally, we remark that, just as in the case of anabelioids, it is crucial in the geometry of temperoids to allow for multiple connected components which arise, for instance, when one pulls back an étale [cf. Definition 3.4, (i) below] morphism of connected temperoids via an arbitrary morphism of connected temperoids.

Remark 3.1.5. It is immediate from the definitions that every temperoid is an almost totally epimorphic category of countably connected type [cf. §0].

Remark 3.1.6. Observe that although every endomorphism of a connected object of an anabelioid is an automorphism, temperoids do not, in general, satisfy this property. Indeed, if $\Pi$ is a [discrete] free group on generators $e_{1}, e_{2}$, and $H \subseteq \Pi$ is the subgroup generated by elements of the form $e_{2}^{n} \cdot e_{1} \cdot e_{2}^{-n}$, where $n$ ranges over the positive integers, then conjugation by $e_{2}$ determines an endomorphism $H \rightarrow H$ [i.e., an endomorphism of the object determined by the $\Pi$-set $\Pi / H$ of $\left.\mathcal{B}^{\text {temp }}(\Pi)\right]$ which is not an automorphism. Nevertheless, it is immediate from the definitions that every endomorphism of a Galois connected object of a temperoid is an automorphism.

Remark 3.1.7. In some situations, instead of considering temperoids or anabelioids, it is useful to consider slightly more general versions of these notions, which we shall refer to as "quasi-temperoids", "quasi-anabelioids", respectively. For more on these "routine" generalizations, we refer to the Appendix.

Remark 3.1.8. For a more general treatment of "categories that behave like anabelioids and temperoids", we refer to [Dub].

Proposition 3.2 (The "Grothendieck Conjecture" for Connected Temperoids). For $i=1,2$, let $\Pi_{i}$ be a tempered [topological] group; write $\mathcal{T}_{i} \stackrel{\text { def }}{=}$ $\mathcal{B}^{\text {temp }}\left(\Pi_{i}\right)$. Then the category of morphisms

$$
\mathcal{T}_{1} \rightarrow \mathcal{T}_{2}
$$


is equivalent to the category whose objects are continuous group homomorphisms $\phi: \Pi_{1} \rightarrow \Pi_{2}$ and whose morphisms $\phi \rightarrow \psi$ are elements $g \in \Pi_{2}$ such that $\gamma_{g} \circ \phi=\psi$, where we write $\gamma_{g}$ for the automorphism of $\Pi_{2}$ given by conjugating by $g$. In particular, there is a natural bijective correspondence between the set of isomorphism classes of morphisms $\mathcal{T}_{1} \rightarrow \mathcal{T}_{2}$ and the set of [continuous] outer homomorphisms $\Pi_{1} \rightarrow \Pi_{2}$.

Proof. Observe that, by thinking of a Galois object $T \in \mathrm{Ob}\left(\mathcal{T}_{2}\right)$ as an "Aut $\mathcal{T}_{2}(T)$-torsor object" [cf. Remark 3.1.3], it follows that if $\phi: \mathcal{T}_{1} \rightarrow \mathcal{T}_{2}$ is a morphism of temperoids, then the object $\phi^{*}(T)$ of $\mathcal{T}_{1}$ is Galois. In light of this observation, the proof of Proposition 3.2 is formally entirely similar to that of [Mzk4], Proposition 1.1.4 [i.e. the "Grothendieck Conjecture" for connected anabelioids].

Remark 3.2.1. In particular, Proposition 3.2 implies that, if $\mathcal{X}$ is a connected temperoid, then it makes sense to write $\pi_{1}^{\text {temp }}(\mathcal{X})$. We shall refer to this tempered group $\pi_{1}^{\text {temp }}(\mathcal{X})$ [which is well-defined, up to inner automorphism] as the tempered fundamental group of $\mathcal{X}$.

Corollary 3.3 (Rigid Morphisms of Connected Temperoids). We maintain the notation of Proposition 3.2. Let $\phi: \Pi_{1} \rightarrow \Pi_{2}$ be a continuous homomorphism that gives rise to a morphism of temperoids $\mathcal{B}^{\text {temp }}(\phi): \mathcal{T}_{1} \rightarrow \mathcal{T}_{2}$. Then $\mathcal{B}^{\text {temp }}(\phi)$ is rigid if and only if the centralizer $Z_{\Pi_{2}}(\operatorname{Im}(\phi))$ is trivial.

Proof. This is a formal consequence of Proposition 3.2 [cf. [Mzk4], Corollary 1.1.6].

Next, let us observe that, if $T \in \mathrm{Ob}(\mathcal{T})$ is an object of a connected temperoid $\mathcal{T}$, then the category

\section{$\mathcal{T}_{T}$}

forms a temperoid [which is connected if and only if $T$ is connected]. Moreover, forming the product with $T$ yields a functor $\mathcal{T} \rightarrow \mathcal{T}_{T}$ which determines a morphism of temperoids as follows:

$$
\mathcal{T}_{T} \rightarrow \mathcal{T}
$$

Thus, in the spirit of [Mzk4], 1 1.2, we make the following definition:

Definition 3.4. $\quad$ (i) An étale morphism of connected temperoids is a morphism that is abstractly equivalent to a morphism of the form $\mathcal{T}_{T} \rightarrow \mathcal{T}$ considered in the above discussion. An étale morphism of temperoids is a morphism each of whose component morphisms is étale. 
(ii) A continuous homomorphism of tempered groups $\Pi_{1} \rightarrow \Pi_{2}$ will be called relatively temp-slim if, for every open subgroup $H \subseteq \Pi_{1}$, the centralizer $Z_{\Pi_{2}}(\operatorname{Im}(H))$ is trivial. A tempered group will be called temp-slim if its identity morphism is relatively temp-slim. A temperoid will be called temp-slim if and only if each of its connected components is equivalent to the " $\mathcal{B}^{\text {temp }}(-)$ " of some temp-slim tempered group.

Remark 3.4.1. In light of Corollary 3.3 above [cf. also [Mzk4], Corollary 1.1.6], one verifies immediately that a temperoid is slim as a category [cf. $\S 0]$ if and only if it is temp-slim as a temperoid; similarly, an anabelioid is slim as an anabelioid [cf. [Mzk4], Definition 1.2.4, (ii)] if and only if it is slim as a category [cf. §0]; moreover, an anabelioid is slim as an anabelioid if and only if its temperification [cf. Remark 3.1.4] is temp-slim as a temperoid.

Remark 3.4.2. $\quad$ By Corollary 3.3, one may work with relatively tempslim morphisms of temperoids as if they are "morphisms in a category" [not "1-morphisms in a 2-category"]. In particular, if one works under this convention, then the category of étale objects over a given temp-slim temperoid $\mathcal{T}$ forms a category which is equivalent to $\mathcal{T}$ itself [cf. [Mzk4], Proposition 1.2.5]. Moreover, just as in the case of anabelioids [cf. [Mzk4], §1.2], one may work with "pro-temperoids" and hence consider universal coverings [which are pro-temperoids] of a given temp-slim temperoid.

Now we return to semi-graphs of anabelioids. Let $\mathcal{G}$ be a connected, countable [i.e., the underlying semi-graph is countable] semi-graph of anabelioids. If $\mathbb{G}$ has at least one vertex, then let us denote by

$$
\mathcal{B}^{\operatorname{cov}}(\mathcal{G})
$$

the category of objects given by data

$$
\left\{S_{v}, \phi_{e}\right\}
$$

where $v$ (respectively, $e$ ) ranges over the vertices (respectively, edges) of $\mathbb{G}$; for each vertex $v, S_{v} \in \mathrm{Ob}\left(\mathcal{G}_{v}^{\top}\right)$; for each edge $e$, with branches $b_{1}, b_{2}$ abutting to vertices $v_{1}, v_{2}$, respectively, $\phi_{e}:\left\{\left(b_{1}\right)_{*}\right\}^{*} S_{v_{1}} \stackrel{\sim}{\rightarrow}\left\{\left(b_{2}\right)_{*}\right\}^{*} S_{v_{2}}$ is an isomorphism in $\mathcal{G}_{e}^{\top}$, and morphisms given by morphisms [in the evident sense] between such data. If $\mathbb{G}$ has no vertices - and hence precisely one edge $e$, which is necessarily isolated - then we shall write $\mathcal{B}^{\operatorname{cov}}(\mathcal{G}) \stackrel{\text { def }}{=} \mathcal{G}_{e}^{\top}$.

The definition of $\mathcal{B}^{\text {cov }}(\mathcal{G})$ extends immediately to arbitrary semi-graphs of anabelioids, each connected component of which is countable. Moreover, for 
such $\mathcal{G}$, we have a natural full embedding:

$$
\mathcal{B}(\mathcal{G}) \hookrightarrow \mathcal{B}^{\operatorname{cov}}(\mathcal{G})
$$

An object of $\mathcal{B}^{\operatorname{cov}}(\mathcal{G})$ that lies in the essential image of $\mathcal{B}(\mathcal{G})$ will be called finite.

Now observe that we may associate, in a natural way, to any object of $\mathcal{B}^{\text {cov }}(\mathcal{G})$ a morphism of countable semi-graphs of anabelioids:

$$
\mathcal{G}^{\prime} \rightarrow \mathcal{G}
$$

[cf. the discussion of $\S 2$ following Definition 2.1 in the case of $\mathcal{B}(\mathcal{G})$ ].

Definition 3.5. (i) We shall refer to a morphism of semi-graphs of anabelioids $\mathcal{G}^{\prime} \rightarrow \mathcal{G}$ that may be constructed in this way as a covering of semigraphs of anabelioids [so, in the terminology of $\S 2$, coverings of semi-graphs of anabelioids that arise from finite objects of $\mathcal{B}^{\mathrm{cov}}(\mathcal{G})$ determine "finite étale coverings of semi-graphs of anabelioids"].

(ii) Suppose that $\mathcal{G}^{\prime} \rightarrow \mathcal{G}$ satisfies the condition that there exists a finite étale covering $\mathcal{G}^{\prime \prime} \rightarrow \mathcal{G}$ with the property that, for any component [i.e., vertex or edge] $c$ of $\mathbb{G}$, the restriction of $\mathcal{G}^{\prime \prime} \rightarrow \mathcal{G}$ to $\mathcal{G}_{c}$ splits the restriction of $\mathcal{G}^{\prime} \rightarrow \mathcal{G}$ to $\mathcal{G}_{c}$. Then we shall say that the covering $\mathcal{G}^{\prime} \rightarrow \mathcal{G}$, as well as the object of $\mathcal{B}^{\operatorname{cov}}(\mathcal{G})$ that gave rise to this covering, is tempered. Also, we shall write

$$
\mathcal{B}^{\text {temp }}(\mathcal{G}) \subseteq \mathcal{B}^{\text {cov }}(\mathcal{G})
$$

for the full subcategory determined by the tempered objects. Thus, we have natural full embeddings:

$$
\mathcal{B}(\mathcal{G}) \hookrightarrow \mathcal{B}^{\text {temp }}(\mathcal{G}) \hookrightarrow \mathcal{B}^{\text {cov }}(\mathcal{G})
$$

(iii) An arrow $\phi: H_{1} \rightarrow H_{2}$ of $\mathcal{B}^{\operatorname{cov}}(\mathcal{G})$ with connected domain and codomain will be called Galois if, for any two arrows $\psi_{1}, \psi_{2}: K \rightarrow H_{1}$ such that $\phi \circ \psi_{1}=\phi \circ \psi_{2}$, there exists a [unique] automorphism $\alpha \in \operatorname{Aut}_{H_{2}}\left(H_{1}\right)$ of $H_{1}$ over $H_{2}$ such that $\psi_{1}=\alpha \circ \psi_{2}$.

Remark 3.5.1. It is immediate from the definitions that passing to the underlying morphism of semi-graphs yields an equivalence between the datum of a locally trivial covering of the semi-graph of anabelioids $\mathcal{G}$ and the datum of a graph-covering with countable fibers of the semi-graph $\mathbb{G}$. 
Remark 3.5.2. It follows from Remark 2.4.2 that, if $\mathcal{G}$ is totally aloof and verticially slim, then the construction given above of a covering of semigraphs of anabelioids associated to an object of $\mathcal{B}^{\operatorname{cov}}(\mathcal{G})$ determines a natural full embedding of $\mathcal{B}^{\text {cov }}(\mathcal{G})$ into the category of totally aloof, verticially slim semigraphs of anabelioids and locally finite étale morphisms over $\mathcal{G}$.

Now let us assume that the semi-graph of anabelioids $\mathcal{G}$ is connected, countable, quasi-coherent, totally elevated, totally aloof, and verticially slim. Let

$$
\left\{\mathcal{G}^{i} \rightarrow \mathcal{G}\right\}_{i \in I}
$$

be some cofinal [i.e., in $\mathcal{B}(\mathcal{G})$ ] collection of connected finite étale Galois coverings, indexed by a set $I$. Thus, $\widehat{\pi}_{1}(\mathcal{G})$ may be constructed as the inverse limit

$$
\varliminf_{i} \operatorname{Gal}\left(\mathcal{G}^{i} / \mathcal{G}\right)
$$

of the resulting inverse system of finite groups $\operatorname{Gal}\left(\mathcal{G}^{i} / \mathcal{G}\right)$. Let us write

$$
\mathcal{G}^{\infty, i} \rightarrow \mathcal{G}^{i}
$$

for the "combinatorial universal covering" of $\mathcal{G}^{i}$ [i.e., the covering of $\mathcal{G}^{i}$ determined by the universal graph-covering of the underlying semi-graph $\mathbb{G}^{i}$; thus, $\operatorname{Gal}\left(\mathcal{G}^{\infty, i} / \mathcal{G}^{i}\right)$ is a free discrete group $]$. One verifies immediately that $\mathcal{G}^{\infty, i} \rightarrow \mathcal{G}$ is a Galois tempered covering. Then we set:

$$
\pi_{1}^{\text {temp }}(\mathcal{G}) \stackrel{\text { def }}{=} \underbrace{}_{i} \operatorname{Gal}\left(\mathcal{G}^{\infty, i} / \mathcal{G}\right)
$$

Note that $\pi_{1}^{\text {temp }}(\mathcal{G})$ is independent, up to inner automorphism, of the choice of the cofinal system $\left\{\mathcal{G}^{i} \rightarrow \mathcal{G}\right\}_{i \in I}$.

Proposition 3.6 (The Tempered Fundamental Group of a Semi-graph of Anabelioids). Let $\mathcal{G}, \mathcal{G}^{\prime}$ be connected, countable, quasi-coherent, totally elevated, totally aloof, verticially slim semi-graphs of anabelioids. Then:

(i) The topological group $\pi_{1}^{\mathrm{temp}}(\mathcal{G})$ defined above is tempered.

(ii) There is a natural equivalence of categories:

$$
\mathcal{B}^{\text {temp }}\left(\pi_{1}^{\text {temp }}(\mathcal{G})\right) \stackrel{\sim}{\rightarrow} \mathcal{B}^{\text {temp }}(\mathcal{G})
$$

In particular, the category $\mathcal{B}^{\text {temp }}(\mathcal{G})$ is a connected temperoid. We shall refer to $\pi_{1}^{\text {temp }}(\mathcal{G})$ as the tempered fundamental group of $\mathcal{G}$. 
(iii) The full embedding $\mathcal{B}(\mathcal{G}) \hookrightarrow \mathcal{B}^{\text {temp }}(\mathcal{G})$ induces an injection $\pi_{1}^{\text {temp }}(\mathcal{G})$ $\hookrightarrow \widehat{\pi}_{1}(\mathcal{G})$ of topological groups.

(iv) Any morphism of semi-graphs of anabelioids $\mathcal{G}^{\prime} \rightarrow \mathcal{G}$ induces a morphism of temperoids

$$
\mathcal{B}^{\text {temp }}\left(\mathcal{G}^{\prime}\right) \rightarrow \mathcal{B}^{\text {temp }}(\mathcal{G})
$$

[by pulling back tempered coverings of $\mathcal{G}$ to tempered coverings of $\mathcal{G}^{\prime}$ ]. Moreover, if the original morphism of semi-graphs of anabelioids is locally open, then this morphism of temperoids is relatively temp-slim. In particular, the temperoid $\mathcal{B}^{\text {temp }}(\mathcal{G})$ is temp-slim.

(v) Suppose that $\mathcal{G}$ is coherent, and that we are given a tempered covering $\mathcal{G}^{\prime} \rightarrow \mathcal{G}$. Then the resulting morphism of temperoids

$$
\mathcal{B}^{\text {temp }}\left(\mathcal{G}^{\prime}\right) \rightarrow \mathcal{B}^{\text {temp }}(\mathcal{G})
$$

is étale.

Proof. Assertion (i) follows from the definitions and the fact that $\mathcal{G}$ is countable. Next, we consider assertion (ii). First, we observe that, by the definition of a tempered covering, it follows that every connected tempered covering $\mathcal{H} \rightarrow \mathcal{G}$ appears as a subcovering of some $\mathcal{G}^{\infty, i} \rightarrow \mathcal{G}$; in particular, we may choose a factorization $\mathcal{G}^{\infty, i} \rightarrow \mathcal{H} \rightarrow \mathcal{G}$. Thus, in light of the definition of $\mathcal{G}^{\infty, i}$ as the "combinatorial universal covering" of $\mathcal{G}^{i}$ [together with the fact that $\mathcal{G}^{i} \rightarrow \mathcal{G}$ is Galois], it follows immediately that the fiber product covering $\mathcal{G}^{\infty, i} \times{ }_{\mathcal{G}} \mathcal{H}$ [regarded as a covering of $\mathcal{G}^{\infty, i}$ via the projection to the first factor] splits as a covering of $\mathcal{G}^{\infty, i}$. Similarly, [cf. also Remark 3.1.3] the covering $\mathcal{G}^{\infty, i} \times_{\mathcal{G}} \mathcal{G}^{\infty, i}$ [regarded as a covering of $\mathcal{G}^{\infty, i}$ via the projection to the first factor] splits as a covering of $\mathcal{G}^{\infty, i}$. Moreover, the action of $\operatorname{Gal}\left(\mathcal{G}^{\infty, i} / \mathcal{G}\right)$ on the first factor of $\mathcal{G}^{\infty, i} \times{ }_{\mathcal{G}} \mathcal{G}^{\infty, i}$ determines an action of $\operatorname{Gal}\left(\mathcal{G}^{\infty, i} / \mathcal{G}\right)$ on the set of components $\pi_{0}\left(\mathcal{G}^{\infty, i} \times{ }_{\mathcal{G}} \mathcal{G}^{\infty, i}\right)$ which is transitive and free of fixed points [cf. Remark 3.1.3]. On the other hand, since the domain and codomain of the covering $\mathcal{G}^{\infty, i} \rightarrow \mathcal{H}$ are connected, one verifies immediately that the resulting map on sets of connected components

$$
\xi: \pi_{0}\left(\mathcal{G}^{\infty, i} \times_{\mathcal{G}} \mathcal{G}^{\infty, i}\right) \rightarrow \pi_{0}\left(\mathcal{G}^{\infty, i} \times_{\mathcal{G}} \mathcal{H}\right)
$$

is surjective and compatible with the natural actions of $\operatorname{Gal}\left(\mathcal{G}^{\infty, i} / \mathcal{G}\right)$ on the domain and codomain [arising from the first factor of the fiber products]. In particular, the fiber of the element of the codomain of $\xi$ determined by the 
"graph" of the covering morphism $\mathcal{G}^{\infty, i} \rightarrow \mathcal{H}$ is the $H$-orbit, for some subgroup $H \subseteq \operatorname{Gal}\left(\mathcal{G}^{\infty, i} / \mathcal{G}\right)$, of the element of the domain of $\xi$ determined by the "graph" of the identity morphism $\mathcal{G}^{\infty, i} \rightarrow \mathcal{G}^{\infty, i}$. Now forming the quotient of $\mathcal{G}^{\infty, i}$ by $H$ yields an intermediate covering $\mathcal{G}^{\infty, i} \rightarrow \mathcal{G}^{H} \rightarrow \mathcal{G}$ such that the morphism $\mathcal{G}^{H} \rightarrow \mathcal{G}$ admits a factorization [by the definition of $H$ !] $\mathcal{G}^{H} \rightarrow \mathcal{H} \rightarrow \mathcal{G}$. On the other hand, by forming the fiber product with $\mathcal{G}^{\infty, i}$ over $\mathcal{G}$ and looking at the induced morphisms on sets of connected components, we obtain surjections

$$
\pi_{0}\left(\mathcal{G}^{\infty, i} \times{ }_{\mathcal{G}} \mathcal{G}^{\infty, i}\right) \rightarrow \pi_{0}\left(\mathcal{G}^{\infty, i} \times{ }_{\mathcal{G}} \mathcal{G}^{H}\right) \rightarrow \pi_{0}\left(\mathcal{G}^{\infty, i} \times{ }_{\mathcal{G}} \mathcal{H}\right)
$$

the composite of which is equal to $\xi$. Moreover, by the construction of $\mathcal{G}^{H}$, it follows formally that the first of these two surjections factors through the quotient of the set $\pi_{0}\left(\mathcal{G}^{\infty, i} \times{ }_{\mathcal{G}} \mathcal{G}^{\infty, i}\right)$ by the action of $H$; we thus conclude [from the fact that $\xi$ induces a bijection of the quotient of the set $\pi_{0}\left(\mathcal{G}^{\infty, i} \times_{\mathcal{G}} \mathcal{G}^{\infty, i}\right)$ by the action of $H$ onto the set $\left.\pi_{0}\left(\mathcal{G}^{\infty, i} \times{ }_{\mathcal{G}} \mathcal{H}\right)\right]$ that the surjection $\pi_{0}\left(\mathcal{G}^{\infty, i} \times \mathcal{G}\right.$ $\left.\mathcal{G}^{H}\right) \rightarrow \pi_{0}\left(\mathcal{G}^{\infty, i} \times \mathcal{G} \mathcal{H}\right)$ is, in fact, a bijection. But from this, one concludes immediately that the covering morphism $\mathcal{G}^{H} \rightarrow \mathcal{H}$ is an isomorphism. Thus, in summary, we conclude that the covering $\mathcal{H} \rightarrow \mathcal{G}$ may be constructed as the quotient of the covering $\mathcal{G}^{\infty, i} \rightarrow \mathcal{G}$ by the action of $\operatorname{Gal}\left(\mathcal{G}^{\infty, i} / \mathcal{H}\right)$ [i.e., $H$ ]; and [by applying the surjectivity of $\xi$ to the "graphs" of various covering morphisms] that every morphism of coverings [of $\mathcal{G}$ ] from $\mathcal{G}^{\infty, i}$ to $\mathcal{H}$ may be obtained from the original morphism by composing with the action of $\operatorname{Gal}\left(\mathcal{G}^{\infty, i} / \mathcal{G}\right)$. Conversely, one verifies immediately that the quotient of $\mathcal{G}^{\infty, i} \rightarrow \mathcal{G}$ by the action of any subgroup of $\operatorname{Gal}\left(\mathcal{G}^{\infty, i} / \mathcal{G}\right)$ yields a subcovering of $\mathcal{G}^{\infty, i} \rightarrow \mathcal{G}$. This shows that there is a natural equivalence between $\mathcal{B}^{\text {temp }}\left(\operatorname{Gal}\left(\mathcal{G}^{\infty, i} / \mathcal{G}\right)\right)$ and the full subcategory of $\mathcal{B}^{\text {temp }}(\mathcal{G})$ determined by the subcoverings of $\mathcal{G}^{\infty, i} \rightarrow \mathcal{G}$. Passing to the limit over $i$ then completes the proof of assertion (ii).

Assertion (iii) follows from the fact that discrete free groups [such as $\operatorname{Gal}\left(\mathcal{G}^{\infty, i} / \mathcal{G}^{i}\right)$ ] are always residually finite [cf., e.g., Corollary 1.7]. The first sentence of assertion (iv) follows from the definitions; the remainder of assertion (iv) follows, in light of the injection of assertion (iii), formally from Corollary 2.7, (ii). Finally, to verify assertion (v), it suffices to show that the composite of any tempered covering $\mathcal{H}^{\prime} \rightarrow \mathcal{G}^{\prime}$ with the given tempered covering $\mathcal{G}^{\prime} \rightarrow \mathcal{G}$ forms a tempered covering $\mathcal{H}^{\prime} \rightarrow \mathcal{G}$; moreover, [since composites of "combinatorial" [i.e., locally trivial] coverings manifestly form "combinatorial" coverings] we may assume without loss of generality that the covering $\mathcal{H}^{\prime} \rightarrow \mathcal{G}^{\prime}$ is finite étale. But then it follows from the coherence of $\mathcal{G}$ [cf. Definition 2.3, (iii)] that there exists a finite étale covering $\mathcal{H} \rightarrow \mathcal{G}$ whose pull-back to $\mathcal{G}^{\prime}$ splits the restrictions of $\mathcal{H}^{\prime} \rightarrow \mathcal{G}^{\prime}$ to each of the $\mathcal{G}_{c}^{\prime}$ [where $c$ is a component of $\left.\mathbb{G}^{\prime}\right]$. 
Theorem 3.7 (Maximal Compact Subgroups of the Tempered Fundamental Group). Let $\mathcal{G}$ be a connected, countable, quasi-coherent, totally elevated, totally estranged, verticially slim semi-graph of anabelioids. Assume that $\mathbb{G}$ has at least one vertex. Then:

(i) For each vertex $v$ of $\mathcal{G}$, there is a natural continuous, injective outer homomorphism $\widehat{\pi}_{1}\left(\mathcal{G}_{v}\right) \hookrightarrow \pi_{1}^{\mathrm{temp}}(\mathcal{G})$. By abuse of notation, we shall write $\widehat{\pi}_{1}\left(\mathcal{G}_{v}\right)$ for the subgroup [well-defined up to conjugation] determined by the image of this homomorphism. We shall refer to the [necessarily compact!] subgroups of $\pi_{1}^{\text {temp }}(\mathcal{G})$ that arise in this way as the verticial subgroups.

(ii) Let us think of the verticial subgroups as being parametrized by a vertex $v$ of $\mathcal{G}$ and an element of the coset space $\pi_{1}^{\text {temp }}(\mathcal{G}) / \widehat{\pi}_{1}\left(\mathcal{G}_{v}\right)$. Then if $H_{1}$, $\mathrm{H}_{2}$ are verticial subgroups of $\pi_{1}^{\text {temp }}(\mathcal{G})$ that arise from distinct parametrization data, then $H_{1} \bigcap H_{2}$ has infinite index in $H_{1}$. In particular, verticial subgroups that arise from distinct parametrization data are distinct.

(iii) Every compact subgroup of $\pi_{1}^{\mathrm{temp}}(\mathcal{G})$ is contained in at least one verticial subgroup. If a nontrivial compact subgroup of $\pi_{1}^{\text {temp }}(\mathcal{G})$ is contained in more than one verticial subgroup, then it is contained in precisely two verticial subgroups, determined by a compatible system of pairs of vertices of the $\mathbb{G}^{\infty, i}$ [ as $i$ ranges over the elements of I] joined to one another by a single [closed] edge. In particular, in this case, this compact subgroup is contained in the image of some $\widehat{\pi}_{1}\left(\mathcal{G}_{e}\right)$, for some edge e of $\mathbb{G}$. We shall refer to such images of " $\widehat{\pi}_{1}\left(\mathcal{G}_{e}\right)$ 's" as the edge-like subgroups of $\pi_{1}^{\mathrm{temp}}(\mathcal{G})$.

(iv) The maximal compact subgroups of $\pi_{1}^{\text {temp }}(\mathcal{G})$ are precisely the verticial subgroups. The nontrivial intersections of two distinct maximal compact subgroups of $\pi_{1}^{\text {temp }}(\mathcal{G})$ are precisely the edge-like subgroups.

Proof. In light of the injection of Proposition 3.6, (iii), assertion (i) (respectively, (ii)) follows from the definitions; Proposition 2.5, (i) (respectively, Proposition 2.6; Corollary 2.7, (i) [cf. also Remark 2.7.2]).

Now suppose that $H \subseteq \pi_{1}^{\text {temp }}(\mathcal{G})$ is a nontrivial compact subgroup. Then $H$ acts continuously on the semi-graph $\mathbb{G}^{\infty, i}$, for each $i \in I$. Thus, this action factors through a finite quotient. In particular, by Lemma 1.8, (ii), (a), $H$ fixes at least one edge or vertex of the semi-graph $\mathbb{G}^{\infty, i}$. Since the action of $H$ is over $\mathbb{G}$, it follows that if $H$ fixes an edge, then it does not switch the branches of the edge. Since $\mathbb{G}$, hence also $\mathbb{G}^{\infty, i}$, is connected 
and has at least one vertex, it thus follows that every edge of $\mathbb{G}^{\infty, i}$ abuts to at least one vertex. In particular, we conclude that if $H$ fixes an edge of $\mathbb{G}^{\infty, i}$, then it fixes a vertex, i.e., that $H$ always fixes at least one vertex of $\mathbb{G}^{\infty, i}$.

Now suppose that for some cofinal subset $J \subseteq I, H$ fixes $\geq 3$ vertices of $\mathbb{G}^{\infty, j}$, for every $j \in J$. Then by Lemma 1.8, (ii), (c), we conclude that, for every $j \in J, H$ acts trivially on some subjoint of $\mathbb{G}^{\infty, j}$. In particular, $H$ acts trivially on some subjoint of $\mathbb{G}^{j}$. Since the semi-graphs $\mathbb{G}^{j}$ are all finite, we thus conclude that we may choose a compatible system of such subjoints [i.e., on which $H$ acts trivially] of the $\mathbb{G}^{j}$. But this implies [cf. Remark 2.2.1] that $H$ is contained in some conjugate in the profinite fundamental group $\widehat{\pi}_{1}(\mathcal{G})$ of some $\widehat{\pi}_{1}\left(\mathcal{G}_{v}\right)$, and, moreover, that it is in fact contained, for two distinct branches $b$, $b^{\prime}$ abutting to $v$ of edges $e, e^{\prime}$, respectively [where $e$ is not necessarily distinct from $e^{\prime}$, in the intersection of the images of $\widehat{\pi}_{1}\left(\mathcal{G}_{e}\right), \widehat{\pi}_{1}\left(\mathcal{G}_{e^{\prime}}\right)$, via $b, b^{\prime}$. But since $\mathcal{G}$ is assumed to be totally estranged, we thus conclude that $H$ is trivial, in contradiction to our hypotheses.

Thus, in summary, we have shown that for some cofinal subset $J \subseteq I$, $H$ fixes at least one, but no more than two vertices of $\mathbb{G}^{\infty, j}$, for every $j \in J$. Moreover, by Lemma 1.8, (ii), (b), it follows that if $H$ fixes two vertices of $\mathbb{G}^{\infty, j}$, then these two vertices are joined to one another by a single [closed] edge. In particular, by possibly replacing $J$ by some smaller cofinal subset, we may assume that there exists a compatible system of vertices of $\mathbb{G}^{\infty, j}$, for $j \in J$, each of which is fixed by $H$. On the other hand, we may also conclude that there exist at most two such compatible systems. This completes the proof of assertion (iii). Finally, assertion (iv) follows formally from assertions (ii), (iii).

Remark 3.7.1. The notion that

maximal compact subgroups correspond to points

is a recurrent theme in the geometry of group actions. Classical well-known examples of this phenomenon include the theory of symmetric spaces obtained as quotients of a real reductive group by a maximal compact subgroup or, in the $p$-adic case, of $\mathbb{Q}_{p}$-valued points of a reductive group by $\mathbb{Z}_{p}$-valued points. Another example of this sort of situation is the "discrete real section conjecture" of $[\mathrm{Mzk} 5], \S 3.2$. 
Definition 3.8. We shall refer to as quasi-geometric any continuous homomorphism of tempered groups

$$
\Pi_{1} \rightarrow \Pi_{2}
$$

that satisfies the following condition: Any maximal compact subgroup $K_{1} \subseteq \Pi_{1}$ (respectively, nontrivial intersection $K_{1} \bigcap H_{1}$ of two distinct maximal compact subgroups $\left.K_{1}, H_{1} \subseteq \Pi_{1}\right)$ maps surjectively to an open subgroup of some maximal compact subgroup $K_{2} \subseteq \Pi_{2}$ (respectively, of some nontrivial intersection $K_{2} \bigcap H_{2}$ of two distinct maximal compact subgroups $K_{2}, H_{2} \subseteq \Pi_{2}$ ). A quasigeometric morphism of temperoids is a morphism of temperoids each of whose component morphisms arises [cf. Proposition 3.2] from a quasi-geometric continuous homomorphism of tempered groups.

Remark 3.8.1. It is immediate that any isomorphism of temperoids is quasi-geometric.

Corollary 3.9 (Reconstruction of the Underlying Semi-graph of Anabelioids). Let $\mathcal{G}, \mathcal{H}$ be connected, countable, quasi-coherent, totally elevated, totally estranged, verticially slim graphs of anabelioids. Then applying " $\mathcal{B}^{\text {temp }}(-)$ " determines a natural bijective correspondence between locally open morphisms of semi-graphs of anabelioids

$$
\mathcal{G} \rightarrow \mathcal{H}
$$

and quasi-geometric morphisms of temperoids $\mathcal{B}^{\text {temp }}(\mathcal{G}) \rightarrow \mathcal{B}^{\text {temp }}(\mathcal{H})$.

Proof. $\quad$ First, we note that any locally open morphism of semi-graphs of anabelioids $\mathcal{G} \rightarrow \mathcal{H}$ determines a morphism of temperoids $\mathcal{B}^{\text {temp }}(\mathcal{G}) \rightarrow$ $\mathcal{B}^{\text {temp }}(\mathcal{H})$ [cf. Proposition 3.6, (iv)] whose quasi-geometricity follows by "substituting" the equivalences of Theorem 3.7, (iv), into Definition 3.8. Next, we observe that by Proposition 3.2; Definition 3.8; Theorem 3.7, (ii), (iii), any quasi-geometric $\phi: \mathcal{B}^{\text {temp }}(\mathcal{G}) \rightarrow \mathcal{B}^{\text {temp }}(\mathcal{H})$ determines a map from the vertices to $\mathbb{G}$ to the vertices of $\mathbb{H}$ - i.e., by considering the unique [conjugacy class of] verticial subgroup(s) of $\pi_{1}^{\text {temp }}(\mathcal{H})$ that contain(s) the image of a given verticial subgroup of $\pi_{1}^{\text {temp }}(\mathcal{G})$. Similarly, by considering nontrivial intersections of maximal compact subgroups, one obtains [in light of the fact that, since $\mathcal{H}$ is totally elevated and totally aloof, all of the edge-like subgroups of $\pi_{1}^{\text {temp }}(\mathcal{H})$ are infinite] that any quasi-geometric $\phi: \mathcal{B}^{\text {temp }}(\mathcal{G}) \rightarrow \mathcal{B}^{\text {temp }}(\mathcal{H})$ determines a map from the edges to $\mathbb{G}$ to the edges of $\mathbb{H}$ which is compatible with the 
map obtained above on vertices. Thus, in summary, we conclude that a quasigeometric $\phi: \mathcal{B}^{\text {temp }}(\mathcal{G}) \rightarrow \mathcal{B}^{\text {temp }}(\mathcal{H})$ determines a map on the underlying graphs $\mathbb{G} \rightarrow \mathbb{H}$ that is functorial in $\phi$.

Next, we observe that if $\phi: \mathcal{B}^{\text {temp }}(\mathcal{G}) \rightarrow \mathcal{B}^{\text {temp }}(\mathcal{H})$ is quasi-geometric, then any morphism

$$
\phi^{\prime}: \mathcal{B}^{\text {temp }}\left(\mathcal{G}^{\prime}\right) \rightarrow \mathcal{B}^{\text {temp }}\left(\mathcal{H}^{\prime}\right)
$$

induced by $\phi$ between étale coverings of the domain and codomain of $\phi$ [i.e., $\mathcal{G}^{\prime} \rightarrow \mathcal{G}, \mathcal{H}^{\prime} \rightarrow \mathcal{H}$ are tempered coverings $]$ is again quasi-geometric. Indeed, this follows immediately from the characterization of nontrivial maximal compact subgroups (respectively, nontrivial intersections of two distinct maximal compact subgroups) as the verticial subgroups (respectively, edge-like subgroups) cf. Theorem 3.7, (iii), (iv). Thus, we obtain a morphism of graphs $\mathbb{G}^{\prime} \rightarrow \mathbb{H}^{\prime}$, which is functorial in $\mathcal{G}^{\prime}, \mathcal{H}^{\prime}$. Finally, by varying $\mathcal{G}^{\prime}, \mathcal{H}^{\prime}$, we conclude that $\phi$ arises from a morphism of graphs of anabelioids which [again by Theorem 3.7, (iii), (iv)] is manifestly unique and locally open. This completes the proof of Corollary 3.9.

Remark 3.9.1. Suppose that $\mathcal{G}$ is as in Theorem 3.7. Then observe that, if $\mathbb{G}$ is a semi-graph which is not a graph, then the techniques developed here are not sufficient, in general, to reconstruct the open edges of $\mathbb{G}$ from, say, the isomorphism class of the tempered group $\pi_{1}^{\text {temp }}(\mathcal{G})$ - cf. Remark 4.8.1.

Example 3.10. Pointed Stable Curves over $p$-adic Local Fields I. Let $K$ be a finite extension of $\mathbb{Q}_{p} ; \bar{K}$ an algebraic closure of $K ; X_{K}^{\text {log a smooth }}$ $\log$ curve over $K$ [cf. §0]. Let us write $X_{\bar{K}}^{\log } \stackrel{\text { def }}{=} X_{K}^{\log } \times_{K} \bar{K}$;

$$
\pi_{1}^{\mathrm{temp}}\left(X_{K}^{\log }\right)
$$

for the tempered fundamental group of [André], $\S 4$. Thus, $\pi_{1}^{\text {temp }}\left(X_{K}^{\log }\right)$ is a tempered topological group [in the sense of Definition 3.1, (i)] and fits into a natural exact sequence:

$$
1 \rightarrow \pi_{1}^{\mathrm{temp}}\left(X_{\bar{K}}^{\log }\right) \rightarrow \pi_{1}^{\mathrm{temp}}\left(X_{K}^{\log }\right) \rightarrow G_{K} \rightarrow 1
$$

[where $G_{K} \stackrel{\text { def }}{=} \operatorname{Gal}(\bar{K} / K)$; we write $\pi_{1}^{\text {temp }}\left(X_{\bar{K}}^{\log }\right)$ for the geometric tempered fundamental group of $X_{K}^{\log }$, i.e., the tempered fundamental group of $X_{\bar{K}}^{\log } \times \bar{K}$ $(\bar{K})^{\wedge}$; the " $\wedge$ " denotes the $p$-adic completion]. To simplify the notation, let us write:

$$
\Pi \stackrel{\text { def }}{=} \pi_{1}^{\text {temp }}\left(X_{K}^{\log }\right) ; \quad \Delta \stackrel{\text { def }}{=} \pi_{1}^{\text {temp }}\left(X_{\bar{K}}^{\log }\right)
$$


Note that $\Delta$ is also tempered, so we obtain temperoids:

$$
\mathcal{B}^{\text {temp }}(\Pi) ; \quad \mathcal{B}^{\text {temp }}(\Delta)
$$

Now let us write

$$
\mathcal{G} \text { (respectively, } \mathcal{G}^{\mathrm{c}} \text { ) }
$$

for the graph of anabelioids (respectively, semi-graph of anabelioids) determined by the semi-graph of profinite groups [without compact structure!] (respectively, with compact structure) associated to the geometric special fiber of the stable model of $X_{\bar{K}}^{\log }$ [cf. [Mzk3], Appendix] over the ring of integers $\mathcal{O}_{\bar{K}}$ of $\bar{K}$. Note that it follows from the definitions that we have a natural equivalence $\mathcal{B}^{\text {temp }}(\mathcal{G}) \stackrel{\sim}{\rightarrow} \mathcal{B}^{\text {temp }}\left(\mathcal{G}^{\mathrm{c}}\right)$ and a natural full embedding:

$$
\mathcal{B}^{\text {temp }}(\mathcal{G}) \hookrightarrow \mathcal{B}^{\text {temp }}(\Delta)
$$

Now suppose that we are given an exhaustive sequence of open characteristic [hence normal] subgroups of finite index

$$
\cdots \subseteq N_{i} \subseteq \cdots \subseteq \Delta
$$

[where $i$ ranges over the positive integers] of $\Delta$; write $\Delta_{i} \stackrel{\text { def }}{=} \Delta / N_{i}$. Thus, $N_{i}$ determines a finite $\log$ étale covering of $X_{\bar{K}}^{\log }$, whose geometric special fiber gives rise to semi-graphs of anabelioids

$$
\mathcal{G}_{i} ; \quad \mathcal{G}_{i}^{\mathrm{c}}
$$

on which $\Delta_{i}$ acts faithfully. Recall from Example 2.10 that $\mathcal{G}_{i}, \mathcal{G}_{i}^{\mathrm{c}}$ are coherent, totally elevated, totally universally sub-coverticial, totally estranged, and verticially slim. In particular, $\mathcal{G}_{i}$ satisfies the hypotheses of Theorem 3.7, Corollary 3.9 .

Next, let us observe that we obtain a natural compatible system of generalized [cf. Definition 2.11] morphisms of graphs of anabelioids

$$
\mathcal{G}_{i} \rightarrow \mathcal{G}_{j}
$$

[where $i \geq j$ ], which are compatible with the actions of $\Delta_{i}, \Delta_{j}$, as follows: The functor

$$
\operatorname{Cat}\left(\mathbb{G}_{i}\right) \rightarrow \operatorname{Cat}\left(\mathbb{G}_{j}\right)
$$

is obtained by mapping a vertex $v$ (respectively, vertex $v$; edge $e$; edge $e$ ) of $\mathbb{G}_{i}$ to a $(\mathrm{n})$ vertex $v^{\prime}$ (respectively, edge $e^{\prime}$; edge $v^{\prime}$; edge $e^{\prime}$ ) of $\mathbb{G}_{j}$ whenever the map on geometric special fibers between the coverings determined by $\Delta_{i}$, 
$\Delta_{j}$ maps the irreducible component corresponding to $v$ into the irreducible component corresponding to $v^{\prime}$ in such a way that the image of the irreducible component corresponding to $v$ is not equal to a node (respectively, the irreducible component corresponding to $v$ into the node corresponding to $e^{\prime}$; the node corresponding to $e$ to a non-nodal point lying in the irreducible component corresponding to $v^{\prime}$; the node corresponding to $e$ to the node corresponding to $\left.e^{\prime}\right)$. The remainder of the data necessary to define the generalized morphism of graphs of anabelioids $\mathcal{G}_{i} \rightarrow \mathcal{G}_{j}$ is determined naturally by considering the map on geometric special fibers between the coverings determined by $\Delta_{i}, \Delta_{j}$. For a more group-theoretic description of these generalized morphisms $\mathcal{G}_{i} \rightarrow \mathcal{G}_{j}$, we refer to the discussion of Example 5.6 below.

Finally, we observe that these generalized morphisms of graphs of anabelioids induce - by applying " $\mathcal{B}^{\text {temp }}(-)$ " [cf. Remark 2.11.1] — natural morphisms of temperoids

$$
\cdots \rightarrow \mathcal{B}^{\text {temp }}\left(\mathcal{G}_{i}\right) \rightarrow \cdots \rightarrow \mathcal{B}^{\text {temp }}\left(\mathcal{G}_{j}\right) \rightarrow \cdots \rightarrow \mathcal{B}^{\text {temp }}(\mathcal{G})
$$

compatible with the actions of the $\Delta_{i}$, hence also corresponding surjections of tempered groups [where we note that each of the "outer semi-direct product groups" [cf. §0] admits a natural topology with respect to which the resulting topological group is tempered]:

$$
\begin{aligned}
\Delta \rightarrow \cdots & \rightarrow \Delta[i] \stackrel{\text { def }}{=} \pi_{1}^{\text {temp }}\left(\mathcal{G}_{i}\right) \stackrel{\text { out }}{\rtimes} \Delta_{i} \rightarrow \cdots \rightarrow \Delta[j] \stackrel{\text { def }}{=} \pi_{1}^{\text {temp }}\left(\mathcal{G}_{j}\right) \stackrel{\text { out }}{\rtimes} \Delta_{j} \\
& \rightarrow \cdots \rightarrow \pi_{1}^{\text {temp }}(\mathcal{G})
\end{aligned}
$$

Note that each $\Delta[i]$ is temp-slim. [Indeed, this follows from the fact that $\Delta_{i}$ acts faithfully on $\mathcal{G}_{i}$, hence also faithfully on $\mathcal{B}^{\text {temp }}\left(\mathcal{G}_{i}\right)$ [cf., e.g., the injectivity portion of the bijection of Corollary 3.9]; the temp-slimness portion of Proposition 3.6, (iv).] Since $\Delta$ is the inverse limit of the $\Delta[i]$, and $G_{K}$ is slim [cf. e.g., [Mzk3], Theorem 1.1.1], we thus conclude that both $\Delta$ and $\Pi$ are temp-slim.

Remark 3.10.1. We maintain the notation of Example 3.10. Write $\mathcal{B}^{\text {temp }}\left(X_{K}^{\log }\right)$ for the category of tempered coverings of $X_{K}^{\log }\left[\right.$ so $\mathcal{B}^{\text {temp }}\left(X_{K}^{\log }\right)$ is a temperoid whose tempered fundamental group is $\left.\pi_{1}^{\text {temp }}\left(X_{K}^{\log }\right)\right]$. Let $\Sigma$ be a set of prime numbers. Denote by

$$
\mathcal{B}^{\text {temp }}\left(X_{K}^{\log }\right)^{\Sigma} \subseteq \mathcal{B}^{\text {temp }}\left(X_{K}^{\log }\right)
$$

the full subcategory determined by the tempered coverings dominated by coverings which arise as a combinatorial covering [i.e., a covering arising from a graph-covering of the dual graph of the geometric special fiber] of a finite étale 
Galois covering of $X_{K}^{\log }$ whose degree is a $\Sigma$-integer. One verifies immediately that $\mathcal{B}^{\text {temp }}\left(X_{K}^{\log }\right)^{\Sigma}$ is a temperoid. We shall refer to the tempered fundamental group of this temperoid as the pro- $\Sigma$ tempered fundamental group of $X_{K}^{\log }$. Then, as long as $\Sigma$ contains at least one prime $\neq p$, the entire discussion of Example 3.10 may be carried out for the pro- $\Sigma$ tempered fundamental group of $X_{K}^{\log }$. [We leave the routine details to the interested reader.] Also, we observe that the analogue of "G" (respectively, "G $\mathcal{G}$ ") in the pro- $\Sigma$ case is precisely the pro- $\Sigma$ completion [in the sense of Definition 2.9, (ii)] of the semi-graph of anabelioids $\mathcal{G}$ (respectively, $\mathcal{G}^{\mathrm{c}}$ ).

In the case of tempered fundamental groups of pointed stable curves, i.e., Example 3.10, we observe [cf. Remark 3.9.1] that, in the notation of Example 3.10 , not only $\mathcal{G}$, but also the semi-graph of anabelioids $\mathcal{G}^{\mathrm{c}}$ may be reconstructed group-theoretically from the tempered fundamental group:

Corollary 3.11 (Reconstruction of Semi-graphs of Anabelioids Associated to Pointed Stable Curves). For $\square=\alpha, \beta$, let $K_{\square}$ be a finite extension of $\mathbb{Q}_{p_{\square}} ; \bar{K}_{\square}$ an algebraic closure of $K_{\square} ;\left(X_{\square}^{\log }\right)_{K_{\square}}$ a smooth log curve over $K_{\square}[c f . \quad \S 0]$. Let us write $\left(X_{\square}^{\log }\right)_{K_{\square}} \stackrel{\text { def }}{=}\left(X_{\square}^{\log }\right)_{K_{\square}} \times_{K_{\square}} \bar{K}_{\square} ; \Delta[\square] \stackrel{\text { def }}{=}$ $\pi_{1}^{\text {temp }}\left(\left(X_{\square}^{\log }\right)_{\bar{K}_{\square}}\right)$;

$$
\mathcal{G}[\square]\left(\text { respectively, } \mathcal{G}^{\mathrm{c}}[\square]\right)
$$

for the graph of anabelioids (respectively, semi-graph of anabelioids) determined by the semi-graph of profinite groups [without compact structure!] (respectively, with compact structure) associated to the geometric special fiber of the stable model of $\left(X_{\square}^{\mathrm{log}}\right)_{\bar{K}_{\square}}[c f$. [Mzk3], Appendix] over the ring of integers $\mathcal{O}_{\bar{K}_{\square}}$ of $\bar{K}_{\square}[$ cf. Example 3.10]. Then any isomorphism of topological groups

$$
\gamma: \Delta[\alpha] \stackrel{\sim}{\rightarrow} \Delta[\beta]
$$

determines a compatible isomorphism of semi-graphs of anabelioids

$$
\mathcal{G}^{\mathrm{c}}[\alpha] \stackrel{\sim}{\rightarrow} \mathcal{G}^{\mathrm{c}}[\beta]
$$

in a fashion that is functorial with respect to $\gamma$. Moreover, if such a $\gamma$ exists, then $p_{\alpha}=p_{\beta}$.

Proof. $\quad$ Let $\Sigma$ be a set of prime numbers such that $p_{\alpha}, p_{\beta} \notin \Sigma$. Write $\widehat{\Delta}[\square]$ for the profinite completion of $\Delta[\square] ; \Delta[\square]^{\Sigma}$ for the pro- $\Sigma$ tempered fundamental group of Remark 3.10.1; $\widehat{\Delta}[\square]^{\Sigma}$ for the maximal pro- $\Sigma$ quotient of $\widehat{\Delta}[\square] ; \mathcal{G}^{\mathrm{c}}[\square]^{\Sigma}, \mathcal{G}[\square]^{\Sigma}$ for the respective pro- $\Sigma$ completions [in the sense of Definition 2.9, (ii)] of $\mathcal{G}^{\mathrm{c}}[\square], \mathcal{G}[\square]$. Moreover, since Galois coverings of degree a 
$\Sigma$-integer are necessarily admissible [cf., e.g., [Mzk1], §3], it follows that $\Delta[\square]^{\Sigma}$ may be identified with the tempered fundamental group $\pi_{1}^{\text {temp }}\left(\mathcal{G}^{\mathrm{c}}[\square]^{\Sigma}\right) \cong$ $\pi_{1}^{\text {temp }}\left(\mathcal{G}[\square]^{\Sigma}\right)$.

Next, let us observe that the kernel

$$
I_{\Sigma}[\square] \stackrel{\text { def }}{=} \operatorname{Ker}\left(\Delta[\square] \rightarrow \Delta[\square]^{\Sigma}\right)
$$

may be recovered as the kernel $J_{\Sigma}[\square]$ of the natural morphism $\Delta[\square] \rightarrow \widehat{\Delta}[\square]^{\Sigma}$. Indeed, since it follows from the definitions that the morphism $\Delta[\square] \rightarrow \widehat{\Delta}[\square]^{\Sigma}$ factors through $\Delta[\square]^{\Sigma}$, we obtain that $I_{\Sigma}[\square] \subseteq J_{\Sigma}[\square]$. On the other hand, the fact that $J_{\Sigma}[\square] \subseteq I_{\Sigma}[\square]$ follows from the fact that free discrete groups inject into their pro- $\Sigma$ completions [cf. Remark 1.7.1]. This completes the proof of the equality $I_{\Sigma}[\square]=J_{\Sigma}[\square]$.

In particular, we conclude that the quotients

$$
\Delta[\square] \rightarrow \Delta[\square]^{\Sigma} \cong \pi_{1}^{\text {temp }}\left(\mathcal{G}[\square]^{\Sigma}\right)
$$

are compatible with $\gamma$. Thus, by Corollary 3.9, we conclude that $\gamma$ induces a natural, functorial isomorphism of graphs of anabelioids

$$
\mathcal{G}[\alpha]^{\Sigma} \stackrel{\sim}{\rightarrow} \mathcal{G}[\beta]^{\Sigma}
$$

hence, in particular, an isomorphism of graphs $\mathbb{G}[\alpha] \stackrel{\sim}{\rightarrow} \mathbb{G}[\beta]$.

Next, let us observe, that:

(i) The technique used to obtain an isomorphism of graphs of anabelioids in the preceding paragraph may also be applied to open subgroups of finite index $\Delta^{\prime}[\alpha] \subseteq \Delta[\alpha], \Delta^{\prime}[\beta] \subseteq \Delta[\beta]$ that correspond via $\gamma$.

Moreover, let us recall that:

(ii) The decomposition groups of cusps in $\Delta[\square]^{\Sigma}$ are commensurably terminal [cf. [Mzk3], Lemma 1.3.7].

(iii) Every nontrivial image via the natural morphism

$$
\Delta^{\prime}[\square]^{\Sigma} \rightarrow \Delta[\square]^{\Sigma}
$$

associated to some open subgroup of finite index $\Delta^{\prime}[\square] \subseteq \Delta[\square]$ of the decomposition group of a node in $\Delta^{\prime}[\square]^{\Sigma}$ [i.e., in the terminology of Theorem 3.7, an "edge-like subgroup"] is either an open subgroup of an edge-like subgroup of $\Delta[\square]^{\Sigma}$ or an open subgroup of a decomposition group of a cusp in $\Delta[\square]^{\Sigma}$ [but not both, since $\mathcal{G}[\square]^{\Sigma}$ is totally aloof - cf. Example 2.10, Remark 2.4.1]. 
(iv) Every decomposition group of a cusp in $\Delta[\square]^{\Sigma}$ admits an open subgroup that arises as the image via some morphism $\Delta^{\prime}[\square]^{\Sigma} \rightarrow \Delta[\square]^{\Sigma}$ as in (ii) of an edge-like subgroup of $\Delta^{\prime}[\square]^{\Sigma}$.

Indeed, (ii) follows from [Mzk3], Lemma 1.3.7; (iii) is immediate from the definitions. On the other hand, (iv) may be verified as follows: Suppose that

$$
\Delta^{\prime}[\square] \subseteq \Delta[\square]
$$

is an open normal subgroup of finite index that corresponds to a covering of $\left(X_{\square}^{\log }\right)_{\bar{K}_{\square}}$ whose ramification indices at the cusps are prime to $p_{\square}$, but which is ramified over the irreducible component $C_{\square}$ of the special fiber of the stable model of $\left(X_{\square}^{\log }\right)_{\bar{K}_{\square}}$ that contains [the restriction to the special fiber of] the cusp of interest, which we shall denote by $x_{\square}$. [Note that such a $\Delta^{\prime}[\square]$ always exists: Indeed, by passing to a Galois covering of degree a $\Sigma$-integer, one may assume that the normalization of $C_{\square}$ is of genus $\geq 2$; then one verifies immediately that the covering arising from "multiplication by $p_{\square}$ on the Jacobian" satisfies the conditions just stated.] Then the ramification over $C_{\square}$ implies that this covering has $\geq p_{\square}$ distinct cusps lying over $x_{\square}$ which, nevertheless, map to the same point of the normalization of $C_{\square}$ in the [necessarily inseparable — by our ramification assumption!] field extension of its function field determined by the covering. Thus, we conclude that [the restrictions to the special fiber of] these distinct cusps must lie on an irreducible component of the special fiber [of the stable model] of the covering that collapses to [the restriction to the special fiber of] $x_{\square}$ [cf. also [Tama2], Theorem 0.2 , for a more general result concerning the existence of coverings with collapsing irreducible components]. Now, sorting through the definitions, we see that this completes the proof of (iv).

Thus, in summary, it follows formally from (i), (ii), (iii), (iv) that the natural, functorial isomorphism of graphs of anabelioids

$$
\mathcal{G}[\alpha]^{\Sigma} \stackrel{\sim}{\rightarrow} \mathcal{G}[\beta]^{\Sigma}
$$

induced by $\gamma$ extends uniquely to a natural, functorial isomorphism of semigraphs of anabelioids

$$
\mathcal{G}^{\mathrm{c}}[\alpha]^{\Sigma} \stackrel{\sim}{\rightarrow} \mathcal{G}^{\mathrm{c}}[\beta]^{\Sigma}
$$

[which may also be regarded as being induced by $\gamma$ ], hence, in particular, an isomorphism of semi-graphs $\mathbb{G}^{\mathrm{c}}[\alpha] \stackrel{\sim}{\rightarrow} \mathbb{G}^{\mathrm{c}}[\beta]$.

Next, let us observe that, if $\Delta^{\prime}[\alpha] \subseteq \Delta[\alpha], \Delta^{\prime}[\beta] \subseteq \Delta[\beta]$ are normal open subgroups of finite index that correspond via $\gamma$, and we write $\mathbb{G}^{\prime}[\square], \mathcal{G}^{\prime}[\square]$ for 
the corresponding graphs/graphs of anabelioids [without compact structure], then the decomposition group

$$
D_{v} \subseteq \Delta[\square] / \Delta^{\prime}[\square]
$$

determined by a vertex $v$ of $\mathbb{G}^{\prime}[\square]$ acts naturally on the anabelioid $\mathcal{G}^{\prime}[\square]_{v}^{\Sigma}$ [i.e., the pro- $\Sigma$ completion of the anabelioid $\left.\mathcal{G}^{\prime}[\square]_{v}\right]$. Thus, the inertia group

$$
I_{v} \subseteq D_{v}
$$

at $v$ - i.e., the subgroup that acts trivially on this anabelioid - is necessarily of order a power of $p_{\square}$. (Indeed, here we use the easily verified fact that any nontrivial automorphism of an irreducible component of the special fiber [of the stable model of the covering determined by $\left.\Delta^{\prime}[\square]\right]$ induces a nontrivial outer automorphism of the tame pro- $\Sigma$ fundamental group [i.e., where "tame" means that one only allows tame ramification at the nodes and cusps] of the open subscheme of this irreducible component given by taking the complement [in this irreducible component] of the nodes and cusps.) In particular, [since there exist $\Delta^{\prime}[\square]$ for which $I_{v}$ is nontrivial - cf., e.g., the proof of assertion (iv) given above] we obtain that $p_{\alpha}=p_{\beta}$. Thus, in the following, we shall write $p \stackrel{\text { def }}{=} p_{\alpha}=p_{\beta}$.

Next, let us observe that the natural quotient

$$
\Delta[\square] \rightarrow \pi_{1}^{\mathrm{temp}}(\mathcal{G}[\square]) \cong \pi_{1}^{\mathrm{temp}}\left(\mathcal{G}^{\mathrm{c}}[\square]\right)
$$

- i.e., the quotient determined by the "admissible quotient" of $\widehat{\Delta}[\square]$, in the sense of [Mzk3], 2 - may be characterized as follows: A normal open subgroup of finite index $\Delta^{\prime}[\square] \subseteq \Delta[\square]$ arises from this quotient if and only if no irreducible component of the special fiber of the stable model of the corresponding covering collapses in the stable model of $\left(X_{\square}^{\log }\right)_{\bar{K}_{\square}}$, and, moreover, the decomposition groups at the nodes and cusps (respectively, inertia groups at the irreducible components) of the corresponding covering are prime to $p$ (respectively, trivial). Indeed, this follows immediately from well-known "purity of the branch locus" results and the well-known "structure of local fundamental groups of stable curves" [cf., e.g., [Tama2], Lemma 2.1]. Now let us write $\left(\mathbb{G}^{\prime}\right)^{\mathrm{c}}[\square],\left(\mathcal{G}^{\prime}\right)^{\mathrm{c}}[\square]$ for the semi-graph/semigraph of anabelioids [with compact structure!] associated to $\Delta^{\prime}[\square]$. Then observe that this characterization is equivalent to the following "group-theoretic" condition [i.e., condition compatible with $\gamma$ ]: 
The natural map $\Delta^{\prime}[\square]^{\Sigma} \rightarrow \Delta[\square]^{\Sigma}$ is quasi-geometric; the stabilizer $\subseteq$ $\Delta[\square] / \Delta^{\prime}[\square]$ of any edge of the semi-graph $\left(\mathbb{G}^{\prime}\right)^{\mathrm{c}}[\square]$ has order prime to $p$; the stabilizer $\subseteq \Delta[\square] / \Delta^{\prime}[\square]$ of any vertex $v$ of the semi-graph $\left(\mathbb{G}^{\prime}\right)^{\mathrm{c}}[\square]$ acts faithfully on the anabelioid $\left(\mathcal{G}^{\prime}\right)^{\mathrm{c}}[\square]_{v}^{\Sigma}$ [i.e., the pro- $\Sigma$ completion of the anabelioid $\left.\left(\mathcal{G}^{\prime}\right)^{\mathrm{c}}[\square]_{v}\right]$.

Thus, we conclude that $\gamma$ induces an isomorphism

$$
\pi_{1}^{\mathrm{temp}}(\mathcal{G}[\alpha]) \cong \pi_{1}^{\mathrm{temp}}(\mathcal{G}[\beta])
$$

hence, by Corollary 3.9, we conclude that $\gamma$ induces an isomorphism of graphs of anabelioids

$$
\mathcal{G}[\alpha] \stackrel{\sim}{\rightarrow} \mathcal{G}[\beta]
$$

which — by applying the functorial isomorphisms " $\mathbb{G}^{c}[\alpha] \stackrel{\sim}{\rightarrow} \mathbb{G}^{c}[\beta]$ " obtained above to arbitrary normal open subgroups of finite index $\Delta^{\prime}[\square] \subseteq \Delta[\square]$ that arise from the "admissible quotient" — induces a uniquely determined isomorphism of semi-graphs of anabelioids

$$
\mathcal{G}^{\mathrm{c}}[\alpha] \stackrel{\sim}{\rightarrow} \mathcal{G}^{\mathrm{c}}[\beta]
$$

[which is, of course, functorial in $\gamma$ ], as desired.

Remark 3.11.1. $\quad$ Note that for any set of primes $\Sigma$ of cardinality $\geq 3$ [i.e., so that $\Sigma$ contains at least one prime $\neq p_{\alpha}, p_{\beta}$ ], the argument given above also yields a "pro- $\Sigma$ version" of Corollary 3.11, i.e., where one replaces the isomorphism

$$
\gamma: \Delta[\alpha] \stackrel{\sim}{\rightarrow} \Delta[\beta]
$$

in the statement of Corollary 3.11 by an isomorphism

$$
\Delta[\alpha]^{\Sigma} \stackrel{\sim}{\rightarrow} \Delta[\beta]^{\Sigma}
$$

between the respective pro- $\Sigma$ tempered fundamental groups [in the sense of Remark 3.10.1].

Remark 3.11.2. $\quad$ Once one recovers the "admissible quotients" $\Delta[\square] \rightarrow$ $\pi_{1}^{\text {temp }}\left(\mathcal{G}^{\mathrm{c}}[\square]\right)$, one may apply the results of [Tama1] to the various verticial subgroups of $\pi_{1}^{\text {temp }}\left(\mathcal{G}^{\mathrm{c}}[\square]\right)$ to recover, in certain cases, the isomorphism class of the curve determined by the complement of the nodes and cusps in the irreducible component of the special fiber corresponding to this verticial subgroup. 


\section{$\S 4$. Categories of Localizations}

In this $\S$, we consider categories of localizations of a semi-graph of anabelioids satisfying certain conditions.

Let $\mathcal{G}$ be a totally aloof, verticially slim semi-graph of anabelioids [so that we may apply Remark 2.4.2]; $\Gamma$ a finite group of automorphisms of $\mathcal{G}$ [i.e., $\Gamma$ acts faithfully on $\mathcal{G}$ ].

If $\mathbb{H} \rightarrow \mathbb{G}$ is a morphism of semi-graphs, then we shall write $\mathcal{G}_{\mathbb{H}}$ for the semi-graph of anabelioids obtained by pulling back [in the evident sense] the semi-graph of anabelioids structure of $\mathcal{G}$ via $\mathbb{H} \rightarrow \mathbb{G}$. If $v$ (respectively, $e ; b$ ) is $\mathrm{a}(\mathrm{n})$ vertex (respectively, edge; branch of an edge) of $\mathbb{G}$, then we shall write

$$
\mathcal{G}[v] \stackrel{\text { def }}{=} \mathcal{G}_{\mathbb{G}[v]} ; \quad \mathcal{G}[e] \stackrel{\text { def }}{=} \mathcal{G}_{\mathbb{G}[e]} ; \quad \mathcal{G}[b] \stackrel{\text { def }}{=} \mathcal{G}_{\mathbb{G}[b]}
$$

[i.e., relative to the natural morphisms $\mathbb{G}[v] \rightarrow \mathbb{G}, \mathbb{G}[e] \rightarrow \mathbb{G}, \mathbb{G}[b] \rightarrow \mathbb{G}$ of $\S 1]$.

Definition 4.1. $\quad$ (i) We shall say that $\Gamma$ acts piecewise faithfully on $\mathcal{G}$ if every element $\gamma \in \Gamma$ satisfies the following condition: If there exists a vertex $v$ of $\mathbb{G}$ such that $\gamma$ fixes $v$ as well as all of the branches of closed edges of $\mathbb{G}$ that abut to $v$, then $\gamma$ is the identity.

(ii) Any locally trivial morphism of totally aloof, verticially slim semigraphs of anabelioids $\mathcal{G}^{\prime} \rightarrow \mathcal{G}$ whose underlying morphism of semi-graphs is an immersion (respectively, excision; embedding) will also be referred to as an immersion (respectively, excision; embedding), or, alternatively, as an immersive (respectively, excisive; embedding) morphism.

(iii) Let $\mathcal{H}$ be a totally aloof, verticially slim semi-graph of anabelioids. Then we shall refer to as a $(\mathcal{G}, \Gamma)$-structure on $\mathcal{H}$ any $\Gamma$-orbit [relative to the action of $\Gamma$ on $\mathcal{G}$ ] of locally finite étale morphisms of totally aloof, verticially slim semi-graphs of anabelioids $\mathcal{H} \rightarrow \mathcal{G}$. We shall refer to any of the morphisms in this orbit as a structure morphism [relative to this particular $(\mathcal{G}, \Gamma)$-structure on $\mathcal{H}]$. We shall say that a $(\mathcal{G}, \Gamma)$-structure on $\mathcal{H}$ is iso-immersive (respectively, iso-excisive) if some [or, equivalently, every] structure morphism $\mathcal{H} \rightarrow \mathcal{G}$ factors as the composite of an immersion (respectively, excision) $\mathcal{H} \rightarrow \mathcal{G}^{\prime}$ with a finite étale morphism $\mathcal{G}^{\prime} \rightarrow \mathcal{G}$ such that $\mathbb{G}^{\prime}$ is untangled [so the composites of the immersion $\mathbb{H} \rightarrow \mathbb{G}^{\prime}$ with the various $\mathbb{H}[v] \rightarrow \mathbb{H}, \mathbb{H}[e] \rightarrow \mathbb{H}, \mathbb{H}[b] \rightarrow \mathbb{H}$ are all embeddings]. If $\mathcal{H} \rightarrow \mathcal{H}^{\prime}$ is a locally finite étale morphism between totally aloof, verticially slim semi-graphs of anabelioids that are equipped with $(\mathcal{G}, \Gamma)$ structures, then we shall say that this morphism is compatible with the $(\mathcal{G}, \Gamma)$ structures if the composite of this morphism with a structure morphism of $\mathcal{H}^{\prime}$ yields a structure morphism of $\mathcal{H}$. 
(iv) Let $\mathcal{H}$ be a totally aloof, verticially slim semi-graph of anabelioids. Then we shall refer to as a local $(\mathcal{G}, \Gamma)$-structure on $\mathcal{H}$ the datum of a $(\mathcal{G}, \Gamma)$ structure for each $\mathcal{H}[c]$, where $c$ varies among the components [i.e., vertices and edges] of the underlying semi-graph $\mathbb{H}$ satisfying the property that if a branch $b$ of an edge $e$ of $\mathbb{H}$ abuts to a vertex $v$ of $\mathbb{H}$, then the given $(\mathcal{G}, \Gamma)$-structures on $\mathcal{H}[v], \mathcal{H}[e]$ coincide on $\mathcal{H}[b]$. We shall say that a local $(\mathcal{G}, \Gamma)$-structure is isoimmersive (respectively, iso-excisive) if each of its constituent $(\mathcal{G}, \Gamma)$-structures is iso-immersive (respectively, iso-excisive). We shall say that a local $(\mathcal{G}, \Gamma)$ structure is verticially iso-excisive if each of its constituent $(\mathcal{G}, \Gamma)$-structures at a vertex is iso-excisive. If $\mathcal{H} \rightarrow \mathcal{H}^{\prime}$ is a locally finite étale morphism between totally aloof, verticially slim semi-graphs of anabelioids that are equipped with local $(\mathcal{G}, \Gamma)$-structures, then we shall say that this morphism is compatible with the local $(\mathcal{G}, \Gamma)$-structures if each of the induced morphisms $\mathcal{H}[c] \rightarrow \mathcal{H}\left[c^{\prime}\right]$ (where $c$ is a component of $\mathbb{H}$ mapping to a component $c^{\prime}$ of $\left.\mathbb{H}^{\prime}\right)$ is compatible with the given $(\mathcal{G}, \Gamma)$-structures.

Remark 4.1.1. $\quad$ Let $\mathcal{H}$ be a totally aloof, verticially slim semi-graph of anabelioids, equipped with a local $(\mathcal{G}, \Gamma)$-structure. Then each component $c$ of $\mathbb{H}$ determines a well-defined $\Gamma$-orbit of components of $\mathbb{G}$ [by mapping $c$ to $\mathbb{G}$ via a structure morphism]. In particular, if $c$ is an edge, then it makes sense to say that $c$ lies over an open (respectively, closed) edge of $\mathbb{G}$. In this case, we shall say that $c$ is $\mathbb{G}$-open (respectively, $\mathbb{G}$-closed). One verifies immediately that any locally finite étale morphism compatible with given local $(\mathcal{G}, \Gamma)$-structures maps $\mathbb{G}$-open (respectively, $\mathbb{G}$-closed) edges to $\mathbb{G}$-open (respectively, $\mathbb{G}$-closed) edges.

Remark 4.1.2. The reason for working with the action of $\Gamma$ [which at times may appear to be somewhat cumbersome - cf., e.g., Definition 4.1, (iii), (iv)] is to "simulate" a stack-theoretic situation, of the sort which occurs naturally, for instance, when one considers formal localizations of pointed stable "orbicurves" [cf. the discussion in the Introduction]. In particular, this circumvents the need to introduce [conceivably even more cumbersome] notions of "orbi-semi-graphs of anabelioids", etc.

Next, we assume further that $\Gamma$ acts piecewise faithfully on $\mathcal{G}$, and that $\mathcal{G}$ is finite, connected, coherent, totally elevated, and totally universally subcoverticial. Then we may define a category of localizations of $\mathcal{G}$ 
associated to the pair $(\mathcal{G}, \Gamma)$ as follows: The (finite) closed objects are the connected finite étale coverings $\mathcal{G}^{\prime}$ of $\mathcal{G}$, which we regard as being equipped with the resulting $\mathcal{G}$ - [i.e., $(\mathcal{G},\{1\})$-)] structure. The infinite open objects are the semigraphs of anabelioids $\mathcal{G}^{\prime \prime}$ that appear as connected tempered coverings of $\mathcal{G}$ of infinite degree. We regard infinite open objects as being equipped with the resulting $\mathcal{G}$-structure. An object that is either closed or infinite open will be called tempered. A finite open object $\mathcal{H}$ is a finite, connected, quasi-coherent, totally elevated, totally universally sub-coverticial, totally aloof, verticially slim semigraph of anabelioids, equipped with an iso-immersive, verticially iso-excisive local $(\mathcal{G}, \Gamma)$-structure, such that $\mathbb{H}$ contains at least one non-isolated open edge which is, however, $\mathbb{G}$-closed. The morphisms between tempered objects (respectively, morphisms from a finite open object to an arbitrary object) are the locally finite étale morphisms of semi-graphs of anabelioids compatible with the $\mathcal{G}$-structure (respectively, local $(\mathcal{G}, \Gamma)$-structure). There are no morphisms from a tempered to a finite open object. This completes the definition of the category $\mathfrak{L o c}(\mathcal{G}, \Gamma)$.

Definition 4.2. $\quad$ (i) The (possibly infinite) verticial length (respectively, edge-wise length) of an object of $\mathfrak{L o c}(\mathcal{G}, \Gamma)$ is defined to be the cardinality of the set of vertices (respectively, closed edges) of the underlying semi-graph. An open object of verticial length 1 (respectively, 2) and edge-wise length 0 (respectively, 1) will be referred to as a nuclear object (respectively, link). A locally trivial morphism from a nuclear object to a link (respectively, an arbitrary object) will be referred to as an $N L$-morphism (respectively, a verticial morphism). The verticial degree of an arrow $\mathcal{H} \rightarrow \mathcal{K}$ in $\mathfrak{L o c}(\mathcal{G}, \Gamma)$ at a vertex $v$ of $\mathbb{H}$ mapping to a vertex $w$ of $\mathbb{K}$ is defined to be the [necessarily finite] degree of the finite étale morphism of anabelioids $\mathcal{H}_{v} \rightarrow \mathcal{K}_{w}$ induced by the arrow.

(ii) A graph-localization morphism in $\mathfrak{L o c}(\mathcal{G}, \Gamma)$ is defined to be a locally trivial morphism which satisfies the condition that it is an isomorphism whenever its domain is closed. A strict graph-localization morphism is a graphlocalization morphism for which the induced arrow on underlying semi-graphs is injective on vertices.

Remark 4.2.1. $\quad$ Note that a morphism in $\mathfrak{L o c}(\mathcal{G}, \Gamma)$ is locally trivial if and only if it is excisive. [Indeed, sufficiency follows from the definition of the term "excisive" [cf. Definition 4.1, (ii)]; as for necessity, the "local surjectivity" of branches follows from the verticial iso-excisiveness of the local $(\mathcal{G}, \Gamma)$-structures involved; the "local injectivity" of branches follows, in light of 
the local triviality assumption, from the total aloofness of the semi-graphs of anabelioids involved.]

Remark 4.2.2. One verifies immediately from the definitions that every embedding in $\mathfrak{L o c}(\mathcal{G}, \Gamma)$ is a strict graph-localization morphism. The converse to this statement, however, may easily be seen to be false in general.

Proposition 4.3 (Basic Properties of the Category of Localizations).

(i) The underlying semi-graph of anabelioids of an object of $\mathfrak{L o c}(\mathcal{G}, \Gamma)$ is connected, coherent, totally elevated, totally universally subcoverticial, totally aloof, verticially slim, and of injective type; if, moreover, this object is finite open, then it is of positive verticial length. The underlying morphism of semi-graphs of a morphism of $\mathfrak{L o c}(\mathcal{G}, \Gamma)$ is locally finite étale.

(ii) If $\mathcal{H}$ is a finite open object of $\mathfrak{L o c}(\mathcal{G}, \Gamma)$, then any excision $\mathbb{H}^{\prime} \rightarrow \mathbb{H}$ of finite connected graphs of positive verticial length determines an excision $\mathcal{H}^{\prime} \stackrel{\text { def }}{=} \mathcal{H}_{\mathbb{H}^{\prime}} \rightarrow \mathcal{H}$ of $\mathfrak{L o c}(\mathcal{G}, \Gamma)$.

(iii) Let $\phi: \mathcal{H} \rightarrow \mathcal{K}$ be a morphism in $\mathfrak{L o c}(\mathcal{G}, \Gamma)$ from a finite open object $\mathcal{H}$ to a tempered object $\mathcal{K}$. Then $\phi$ is not an isomorphism of semi-graphs of anabelioids.

(iv) Let $\mathcal{H}$ be a finite open object of $\mathfrak{L o c}(\mathcal{G}, \Gamma)$ such that $\mathbb{H}$ is a tree. Then the local $(\mathcal{G}, \Gamma)$-structure on $\mathcal{H}$ arises from a unique $(\mathcal{G}, \Gamma)$-structure on $\mathcal{H}$ [hence, in particular, from a [not necessarily unique] $\mathcal{G}$-structure].

(v) Every morphism in $\mathfrak{L o c}(\mathcal{G}, \Gamma)$ is an epimorphism. In particular, the category $\mathfrak{L o c}(\mathcal{G}, \Gamma)$ is totally epimorphic.

(vi) Every endomorphism of a finite [open or closed] object of $\mathfrak{L o c}(\mathcal{G}, \Gamma)$ is an automorphism. Moreover, the automorphism group of any finite object of $\mathfrak{L o c}(\mathcal{G}, \Gamma)$ is finite.

Proof. Assertion (i) (respectively, (ii)) is immediate from the definitions [cf. also Remark 2.4.1] (respectively, [cf. also the fact that the domain of any nonproper excision admits an edge that maps to an edge of strictly greater verticial cardinality]). As for assertion (iii), we may assume without loss of generality that $\mathcal{K}$ is finite. Then assertion (iii) follows from the fact that $\phi$ 
necessarily maps an open, $\mathbb{G}$-closed edge of $\mathbb{H}$ [which always exists, by the definition of an open object] to an open, $\mathbb{G}$-closed edge of $\mathbb{K}$. But this contradicts the easily verified fact that every $\mathbb{G}$-closed edge of $\mathbb{K}$ is closed in $\mathbb{K}$.

Next, we consider assertion (iv). The desired morphism $\mathcal{H} \rightarrow \mathcal{G}$ may be constructed by gluing together local structure morphisms to $\mathcal{G}$; the fact that such a gluing operation may be performed — despite the " $\Gamma$-ambiguities" involved - follows from our assumption that $\mathbb{H}$ is a tree. Finally, the uniqueness of the $(\mathcal{G}, \Gamma)$-structure follows by reducing to the case of nuclear $\mathcal{H}$ by assertion (v) below [one checks immediately that there are no vicious circles in the argument], in which case the desired uniqueness is immediate from the definitions.

Next, we consider assertion (v). Let $\phi, \psi: \mathcal{H} \rightarrow \mathcal{K}, \xi: \mathcal{H}^{\prime} \rightarrow \mathcal{H}$ be morphisms in $\mathfrak{L o c}(\mathcal{G}, \Gamma)$ such that $\phi \circ \xi=\psi \circ \xi$. By localizing on $\mathbb{H}$ [and applying the fact that any finite étale morphism of slim anabelioids is an epimorphism in the category of finite étale morphisms of slim anabelioids], one verifies immediately that it suffices to treat the case where $\xi$ is an $N L$-morphism [so, in particular, $\mathcal{H}^{\prime}$ is nuclear; $\mathcal{H}$ is a link]. Write $e_{H}$ for the unique closed edge of $\mathbb{H} ; b_{H}$ for the branch of $e_{H}$ that abuts to the unique vertex $v$ of $\mathbb{H}^{\prime}$. Since $\phi, \psi$ then coincide on the edges of $\mathbb{H}$ that abut to $v$, we conclude that $\phi, \psi$ coincide on $\mathbb{H}\left[e_{H}\right]$. In particular, we may also assume that $\mathcal{K}$ is a link. Write $e_{K}$ for the unique closed edge of $\mathbb{K}$.

Now I claim that it suffices to show that $\phi, \psi$ coincide on $\mathcal{H}\left[e_{H}\right]$. Indeed, since $\mathcal{H}$ is a link, $\mathcal{H}\left[e_{H}\right]$ may be obtained from $\mathcal{H}$ by simply omitting the open edges. Thus, it suffices to check that $\phi, \psi$ map each open edge $e^{\prime}$ of $\mathbb{H}$ to the same open edge $e^{\prime \prime}$ of $\mathbb{K}$ and induce the same morphism $\mathcal{H}_{e^{\prime}} \rightarrow \mathcal{K}_{e^{\prime \prime}}$. On the other hand, since $\phi, \psi$ coincide on $\mathcal{H}\left[e_{H}\right]$, both of these assertions follow from the fact that $\mathcal{K}$ is totally aloof. This completes the proof of the claim.

To show that $\phi, \psi$ coincide on $\mathcal{H}\left[e_{H}\right]$, we reason as follows: First, we observe that [by our definition of the category $\mathfrak{L o c}(\mathcal{G}, \Gamma)$; the piecewise faithfulness of $\Gamma] \mathcal{H}\left[b_{H}\right] ; \mathcal{H}\left[e_{H}\right] ; \mathcal{K}\left[e_{K}\right]$ may be equipped with $\mathcal{G}$-structures that are compatible with $\xi, \phi$, and $\psi$. Thus, these $\mathcal{G}$-structures induce injective [by Proposition 2.5, (i)] outer homomorphisms

$$
\begin{gathered}
\widehat{\pi}_{1}\left(\mathcal{H}^{\prime}\right)=\widehat{\pi}_{1}\left(\mathcal{H}\left[b_{H}\right]\right) \hookrightarrow \widehat{\pi}_{1}(\mathcal{G}) ; \\
\widehat{\pi}_{1}(\mathcal{H})=\widehat{\pi}_{1}\left(\mathcal{H}\left[e_{H}\right]\right) \hookrightarrow \widehat{\pi}_{1}(\mathcal{G}) ; \quad \widehat{\pi}_{1}(\mathcal{K})=\widehat{\pi}_{1}\left(\mathcal{K}\left[e_{K}\right]\right) \hookrightarrow \widehat{\pi}_{1}(\mathcal{G})
\end{gathered}
$$

which are compatible with the outer homomorphisms

$$
\widehat{\pi}_{1}(\xi): \widehat{\pi}_{1}\left(\mathcal{H}^{\prime}\right) \rightarrow \widehat{\pi}_{1}(\mathcal{H}) ; \quad \widehat{\pi}_{1}(\phi), \widehat{\pi}_{1}(\psi): \widehat{\pi}_{1}(\mathcal{H}) \rightarrow \widehat{\pi}_{1}(\mathcal{K})
$$

induced by $\xi, \phi, \psi$. This compatibility implies that $\widehat{\pi}_{1}(\phi), \widehat{\pi}_{1}(\psi)$ differ by conjugation by some element $g \in \widehat{\pi}_{1}(\mathcal{G})$. By the coincidence of $\phi, \psi$ on $\mathcal{H}^{\prime}$, 
however, we may assume that $g$ centralizes [the image of] $\widehat{\pi}_{1}\left(\mathcal{H}^{\prime}\right)$. Thus, by Corollary 2.7, (i), we conclude that $g$ is the identity, and hence that $\widehat{\pi}_{1}(\phi)$, $\widehat{\pi}_{1}(\psi)$ coincide. On the other hand, by Corollary 2.7 , (i), and the fact that $\mathcal{K}$ is totally aloof, this implies that $\phi, \psi$ coincide on $\mathcal{H}\left[e_{H}\right]$, as desired.

Finally, we consider assertion (vi). Let $\phi$ be an endomorphism of a finite [open or closed] object $\mathcal{H}$. By finiteness, it is immediate that, for some integer $M \geq 1, \phi^{M}$ fixes some vertex $v$ of $\mathcal{H}$ and induces the identity on the anabelioid $\mathcal{H}_{v}$. Thus, by assertion (v), we conclude that $\phi^{M}$ is the identity, so $\phi$ is an automorphism, as desired. The finiteness of the automorphism group of $\mathcal{H}$ is immediate from the finiteness of $\mathcal{H}$ itself.

Proposition 4.4 (Associated Anabelioids). Let $\mathcal{H} \rightarrow \mathcal{K}, \mathcal{L} \rightarrow \mathcal{K}$ be morphisms between finite [open or closed] objects in $\mathfrak{L o c}(\mathcal{G}, \Gamma)$. Then:

(i) There exists a finite étale covering $\mathcal{K}^{\prime} \rightarrow \mathcal{K}$ of $\mathcal{K}$ such that the induced morphism $\mathcal{H}^{\prime} \rightarrow \mathcal{K}^{\prime}$ from any connected component $\mathcal{H}^{\prime}$ of the pull-back of this finite étale covering to $\mathcal{H}$ is an embedding.

(ii) There exists a finite étale covering $\mathcal{K}^{\prime} \rightarrow \mathcal{K}$ of $\mathcal{K}$ such that $\mathcal{K}^{\prime}$ embeds into a finite étale covering $\mathcal{G}^{\prime} \rightarrow \mathcal{G}$ of $\overline{\mathcal{G}}$.

(iii) The morphism $\mathcal{H} \rightarrow \mathcal{K}$ in $\mathfrak{L o c}(\mathcal{G}, \Gamma)$ induces a relatively slim $\pi_{1}$ mono-morphism of slim anabelioids

$$
\mathcal{B}(\mathcal{H}) \rightarrow \mathcal{B}(\mathcal{K})
$$

which completely determines the original morphism $\mathcal{H} \rightarrow \mathcal{K}[$ among all morphisms in $\mathfrak{L o c}(\mathcal{G}, \Gamma)$ from $\mathcal{H}$ to $\mathcal{K}]$.

(iv) Suppose that $\mathcal{H} \rightarrow \mathcal{K}, \mathcal{L} \rightarrow \mathcal{K}$ are finite étale. Then every morphism $\mathcal{H} \rightarrow \mathcal{L}$ in $\mathfrak{L o c}(\mathcal{G}, \Gamma)$ lying over $\mathcal{K}$ is finite étale and induces a finite étale morphism on associated anabelioids [cf. assertion (iii)]. Moreover, the full subcategory of such finite étale objects over $\mathcal{K}$ determines a full embedding:

$$
\mathcal{B}(\mathcal{K})^{0} \hookrightarrow \mathfrak{L o c}(\mathcal{G}, \Gamma)_{\mathcal{K}}
$$

When $\mathcal{K}=\mathcal{G}$, we have $\mathfrak{L o c}(\mathcal{G}, \Gamma)_{\mathcal{K}}=\mathfrak{L o c}(\mathcal{G}, \Gamma)$; the essential image of this embedding is the full subcategory of closed objects.

Proof. First, we consider assertion (i). Since $\mathcal{K}$ is quasi-coherent, it follows from Proposition 2.5, (i), that we may reduce immediately to the case where the given morphism $\mathcal{H} \rightarrow \mathcal{K}$ is locally trivial. Thus, we are reduced to 
a problem in graph theory - a problem solved in $\S 1$ - cf. Theorem 1.2 [i.e., "Zariski's main theorem for semi-graphs"].

Assertion (ii) may be shown as follows: Let $\mathcal{K}^{\prime \prime} \rightarrow \mathcal{K}$ be a combinatorial universal covering. Although $\mathcal{K}^{\prime \prime}\left[\right.$ i.e., $\mathcal{K}^{\prime \prime} \rightarrow \mathcal{K}$ ] will not, in general, determine an object of $\mathfrak{L o c}(\mathcal{G}, \Gamma)_{\mathcal{K}}$, it can, nevertheless, be thought of as inductive limit of $\mathcal{K}_{\alpha} \in \operatorname{Ob}\left(\mathfrak{L o c}(\mathcal{G}, \Gamma)_{\mathcal{K}}\right)$ associated to connected finite sub-semi-graphs $\mathbb{K}_{\alpha}$ of $\mathbb{K}^{\prime \prime}$. By Proposition 4.3, (iv), these $\mathcal{K}_{\alpha}$ admit compatible [i.e., as $\alpha$ varies] morphisms [in $\mathfrak{L o c}(\mathcal{G}, \Gamma)_{\mathcal{K}}$ ] to $\mathcal{G}$. Moreover, the uniqueness, up to finitely many possibilities, [cf. Proposition 4.3, (iv)] of such a compatible system implies that some finite index subgroup of $\operatorname{Gal}\left(\mathcal{K}^{\prime \prime} / \mathcal{K}\right)$ fixes such a compatible system. In particular, we conclude that, for some finite subcovering $\mathcal{K}^{\prime \prime \prime} \rightarrow \mathcal{K}$ of $\mathcal{K}^{\prime \prime} / \mathcal{K}$, we obtain a morphism $\left[\right.$ in $\left.\mathfrak{L o c}(\mathcal{G}, \Gamma)_{\mathcal{K}}\right] \mathcal{K}^{\prime \prime \prime} \rightarrow \mathcal{G}$. Thus, the existence of a $\mathcal{K}^{\prime} \rightarrow \mathcal{K}$ as asserted follows by applying assertion (i) to the morphism $\mathcal{K}^{\prime \prime \prime} \rightarrow \mathcal{G}$.

Next, we consider of assertion (iii). The fact that $\mathcal{B}(\mathcal{H}), \mathcal{B}(\mathcal{K})$ are slim follows from Corollary 2.7, (ii). This slimness implies that the profinite fundamental groups of $\mathcal{B}(\mathcal{H}), \mathcal{B}(\mathcal{K})$ have no nontrivial normal finite closed subgroups. Thus, by assertion (i), to show that $\mathcal{B}(\mathcal{H}) \rightarrow \mathcal{B}(\mathcal{K})$ is a $\pi_{1}$-monomorphism, it suffices to show, under the further assumption that $\mathcal{H} \rightarrow \mathcal{K}$ is an embedding, that any finite étale covering $\mathcal{H}^{\prime} \rightarrow \mathcal{H}$ of $\mathcal{H}$ may be split by the pull-back of a finite étale covering $\mathcal{K}^{\prime} \rightarrow \mathcal{K}$ of $\mathcal{K}$; moreover, by the injectivity of Proposition 2.5, (i), we may even assume further that $\mathcal{H}^{\prime} \rightarrow \mathcal{H}$ is locally trivial. But then the existence of a covering $\mathcal{K}^{\prime} \rightarrow \mathcal{K}$ as desired follows from the [easily verified] assertion that "a finite graph-covering of a connected sub-semi-graph of a given connected semi-graph may be extended to a finite graph-covering of the given connected semi-graph" [cf. the proof of Proposition 2.5, (i)]. This completes the proof of the fact that $\mathcal{B}(\mathcal{H}) \rightarrow \mathcal{B}(\mathcal{K})$ is a $\pi_{1}$-monomorphism. Next, we show that $\mathcal{B}(\mathcal{H}) \rightarrow \mathcal{B}(\mathcal{K})$ is relatively slim and determines $\mathcal{H} \rightarrow \mathcal{K}$. By Proposition 4.3 , (v), we reduce immediately to the case where $\mathcal{H}$ is nuclear. Then our conclusion follows from Corollary 2.7, (i), (ii), (iii). This completes the proof of assertion (iii).

Finally, assertion (iv) is a formal consequence of assertion (iii); Proposition 4.3, (iii); and the definitions.

Proposition 4.5 (The Subcategory of Tempered Objects). Let $\mathcal{H}, \mathcal{K}$ be tempered objects of $\mathfrak{L o c}(\mathcal{G}, \Gamma)$. Then every morphism $\mathcal{H} \rightarrow \mathcal{K}$ in $\mathfrak{L o c}(\mathcal{G}, \Gamma)$ is a tempered covering. In particular, we have a natural full embedding

$$
\mathcal{B}^{\text {temp }}(\mathcal{G})^{0} \hookrightarrow \mathfrak{L o c}(\mathcal{G}, \Gamma)
$$

whose essential image is the full subcategory of tempered objects. 
Proof. Indeed, by Proposition 3.6, (iv), (v), we obtain a morphism of temperoids

$$
\mathcal{B}^{\text {temp }}(\mathcal{H}) \rightarrow \mathcal{B}^{\text {temp }}(\mathcal{K})
$$

that is compatible with the étale morphisms of temperoids $\mathcal{B}^{\text {temp }}(\mathcal{H}) \rightarrow$ $\mathcal{B}^{\text {temp }}(\mathcal{G}), \mathcal{B}^{\text {temp }}(\mathcal{K}) \rightarrow \mathcal{B}^{\text {temp }}(\mathcal{G})$ induced by the $\mathcal{G}$-structures. It thus follows formally that the morphism $\mathcal{B}^{\text {temp }}(\mathcal{H}) \rightarrow \mathcal{B}^{\text {temp }}(\mathcal{K})$ is étale, hence corresponds to some tempered covering $\mathcal{H} \rightarrow \mathcal{K}$. But this tempered covering must coincide with the original morphism $\mathcal{H} \rightarrow \mathcal{K}$. Indeed, both morphisms induce the same arrow $\mathcal{B}^{\text {temp }}(\mathcal{H}) \rightarrow \mathcal{B}^{\text {temp }}(\mathcal{K})$. Thus, if $v$ is a vertex of $\mathbb{H}$, and $\mathcal{K}^{\prime} \rightarrow \mathcal{K}$ is an excision with finite open domain such that the restrictions to $\mathcal{H}[v]$ of the two morphisms in question both factor through $\mathcal{K}^{\prime}$, then we conclude that these two morphisms both induce the same arrow $\mathcal{B}\left(\mathcal{H}_{v}\right) \rightarrow \mathcal{B}\left(\mathcal{K}^{\prime}\right)$ [cf. Proposition 2.5, (i); Corollary 2.7, (i)], so we conclude by Proposition 4.3, (v); Proposition 4.4, (iii).

Proposition 4.6 (Valuative Criterion for Finite Étale Morphisms). Let $\phi: \mathcal{H} \rightarrow \mathcal{K}$ be a morphism between finite objects in $\mathfrak{L o c}(\mathcal{G}, \Gamma)$. Then $\phi$ is a finite étale morphism if and only if the following condition is satisfied: for every $\mathbf{N L}$-morphism $\mathcal{H}_{0} \rightarrow \mathcal{H}_{1}$ and every commutative diagram

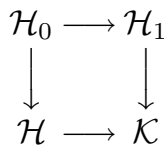

in $\mathfrak{L o c}(\mathcal{G}, \Gamma)$, there exists a commutative diagram

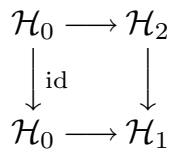

- where the horizontal arrows are NL-morphisms - such that the composite commutative diagram

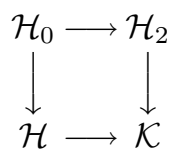

admits a morphism $\mathcal{H}_{2} \rightarrow \mathcal{H}$ in $\mathfrak{L o c}(\mathcal{G}, \Gamma)$ that makes the resulting triangles in this diagram commute.

Proof. First, we consider necessity. By pulling back the finite étale morphism $\mathcal{H} \rightarrow \mathcal{K}$ to $\mathcal{H}_{1}$, we reduce immediately to the case where $\mathcal{H}_{1}=\mathcal{K}$. 
But then the fact that the condition in question is satisfied follows immediately from the definition of a finite étale morphism.

Next, we consider sufficiency. By base-changing $\mathcal{H} \rightarrow \mathcal{K}$ by some finite étale morphism as in Proposition 4.4, (i), we reduce immediately to the case where $\mathcal{H} \rightarrow \mathcal{K}$ is an embedding. But then the condition in question implies that $\mathbb{H} \rightarrow \mathbb{K}$ is surjective, which implies that $\mathcal{H} \rightarrow \mathcal{K}$ is an isomorphism [hence, in particular, finite étale], as desired.

Proposition 4.7 (Domination of Links). For $i=1,2$, let $\mathcal{H}_{0} \rightarrow \mathcal{H}_{i}$ be an NL-morphism. Suppose that the unique closed edge of $\mathbb{H}_{i}$ is the image of the same [i.e., for $i=1,2]$ edge of $\mathbb{H}_{0}$. Then there exists an NL-morphism $\mathcal{H}_{0} \rightarrow \mathcal{H}_{3}$ which fits into commutative diagrams

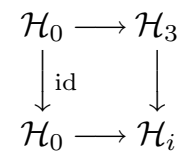

for $i=1,2$.

Proof. $\quad$ First, we observe that we may choose $\mathcal{G}$-structures on $\mathcal{H}_{1}, \mathcal{H}_{2}$ that coincide when restricted to $\mathcal{H}_{0}$ [cf. Proposition 4.3, (iv)]. In the following, " $i$ " will always range over the elements of the set $\{1,2\}$. Now by the definition of $\mathfrak{L o c}(\mathcal{G}, \Gamma)$, there exists a finite Galois étale covering

$$
\mathcal{G}^{\prime} \rightarrow \mathcal{G}
$$

together with finite étale subcoverings $\mathcal{G}^{\prime} \rightarrow \mathcal{G}_{i} \rightarrow \mathcal{G}$ [for $\left.i=1,2\right]$ such that $\mathbb{G}^{\prime}$, $\mathbb{G}_{i}$ are untangled; there exists an embedding $\mathcal{H}_{i} \rightarrow \mathcal{G}_{i}$ compatible with the $\mathcal{G}$ structures. Then [by conjugating $\mathcal{G}_{i}$ appropriately] we may assume that there exists a vertex $v^{\prime}$ (respectively, edge $e^{\prime}$ ) of $\mathcal{G}^{\prime}$ whose image in $\mathcal{G}_{i}$ is equal to the image of the unique vertex of $\mathcal{H}_{0}$ (respectively, the unique closed edge of $\mathcal{H}_{i}$ ) via the composite morphism $\mathcal{H}_{0} \rightarrow \mathcal{H}_{i} \rightarrow \mathcal{G}_{i}$ (respectively, $\mathcal{H}_{i} \rightarrow \mathcal{G}_{i}$ ), and, moreover, that $I \bigcap H_{1}=I \bigcap H_{2}$, where we write

$$
H_{i} \subseteq \operatorname{Gal}\left(\mathcal{G}^{\prime} / \mathcal{G}\right)
$$

for the subgroup determined by the subcovering $\mathcal{G}_{i} \rightarrow \mathcal{G}$ and

$$
I \subseteq \operatorname{Gal}\left(\mathcal{G}^{\prime} / \mathcal{G}\right)
$$

for the isotropy subgroup associated to $v^{\prime}$. Set $H_{3} \stackrel{\text { def }}{=} I \bigcap H_{1}=I \bigcap H_{2}$; write $\mathcal{G}_{3} \rightarrow \mathcal{G}$ for the subcovering determined by $H_{3}$. Then if we take $\mathcal{H}_{3}$ to be 
the link contained in $\mathcal{G}_{3}$ which is determined by the images $v_{3}, e_{3}$ of $v^{\prime}, e^{\prime}$, respectively, then the natural morphism $\mathcal{G}_{3} \rightarrow \mathcal{G}_{i}$ restricts to a morphism

$$
\mathcal{H}_{3} \rightarrow \mathcal{H}_{i}
$$

with the desired properties.

Remark 4.7.1. $\quad$ Note that by applying Proposition 4.7 in an iterative fashion, one may construct an $N L$-morphism with domain $\mathcal{H}_{0}$ that dominates an arbitrary given finite collection of NL-morphisms $\mathcal{H}_{0} \rightarrow \mathcal{H}_{i}$, for $i=1, \ldots, n$ [i.e., such that the unique closed edge of each $\mathbb{H}_{i}$ is the image of the same edge of $\mathbb{H}_{0}$ ]. Moreover, given any pair of such dominating NL-morphisms $\mathcal{H}_{0} \rightarrow \mathcal{H}_{a}$, $\mathcal{H}_{0} \rightarrow \mathcal{H}_{b}$, there exists [again by Proposition 4.7] an NL-morphism $\mathcal{H}_{0} \rightarrow \mathcal{H}_{c}$ that dominates $\mathcal{H}_{0} \rightarrow \mathcal{H}_{a}, \mathcal{H}_{0} \rightarrow \mathcal{H}_{b}$. Observe that in this situation, although $a$ priori, we obtain two arrows $\mathcal{H}_{c} \rightarrow \mathcal{H}_{i}$ [i.e., one passing through $\mathcal{H}_{a}$, the other passing through $\mathcal{H}_{b}$ ], these two arrows necessarily coincide [by Proposition 4.3, (v)]. Thus, in summary, for each edge $e$ of $\mathcal{H}_{0}$, we obtain a natural system of dominating $N L$-morphisms with domain $\mathcal{H}_{0}$, each of whose codomains is a link with closed edge given by the image [via the NL-morphism under consideration] of $e$.

Theorem 4.8 (Category-Theoreticity of Categories of Localizations). For $i=1,2$, let $\mathcal{G}_{i}$ be a finite, connected, coherent, totally elevated, totally universally sub-coverticial, totally aloof, verticially slim graph of anabelioids, with underlying graph $\mathbb{G}_{i}$; let $\Gamma_{i}$ be a finite group that acts piecewise faithfully on $\mathcal{G}_{i}$. Suppose that $\mathbb{G}_{i}$ has at least one edge. Write

$$
\mathfrak{L o c}\left(\mathcal{G}_{i}, \Gamma_{i}\right)^{\mathrm{fin}} \subseteq \mathfrak{L o c}\left(\mathcal{G}_{i}, \Gamma_{i}\right)
$$

for the full subcategory determined by the finite objects. Then the categories $\mathfrak{L o c}\left(\mathcal{G}_{i}, \Gamma_{i}\right)^{\text {fin }}$ (respectively, $\left.\mathfrak{L o c}\left(\mathcal{G}_{i}, \Gamma_{i}\right)\right)$ are $\mathbf{s l i m}$; every equivalence of categories

$$
\begin{aligned}
& \Phi: \mathfrak{L o c}\left(\mathcal{G}_{1}, \Gamma_{1}\right) \text { fin } \stackrel{\sim}{\rightarrow} \mathfrak{L o c}\left(\mathcal{G}_{2}, \Gamma_{2}\right)^{\text {fin }} \\
&\text { respectively, } \left.\Phi: \mathfrak{L o c}\left(\mathcal{G}_{1}, \Gamma_{1}\right) \stackrel{\sim}{\rightarrow} \mathfrak{L o c}\left(\mathcal{G}_{2}, \Gamma_{2}\right)\right)
\end{aligned}
$$

arises, up to unique isomorphism, from a unique isomorphism of graphs of anabelioids

$$
\mathcal{G}_{1} \stackrel{\sim}{\rightarrow} \mathcal{G}_{2}
$$

together with a compatible isomorphism $\Gamma_{1} \stackrel{\sim}{\rightarrow} \Gamma_{2}$. 
Proof. First, we reconstruct the underlying semi-graph of anabelioids of an object $\mathcal{H}$ of $\mathcal{C} \stackrel{\text { def }}{=} \mathfrak{L o c}\left(\mathcal{G}_{i}, \Gamma_{i}\right)$ fin (respectively, $\mathcal{C} \stackrel{\text { def }}{=} \mathfrak{L o c}\left(\mathcal{G}_{i}, \Gamma_{i}\right)$ ) [where $i=1,2]$ category-theoretically as follows: The objects of verticial length 1 are precisely the indissectible [cf. $\S 0]$ objects. An object $\mathcal{H}$ of verticial length 1 is nuclear if and only if the domain of every morphism with codomain $\mathcal{H}$ is of verticial length 1 . If $\mathcal{H}$ is nuclear, then the result of applying " $\perp$ " to the category $\mathcal{C}_{\mathcal{H}}$ of objects and morphisms over $\mathcal{H}$ is a Galois category isomorphic to the anabelioid $\mathcal{B}(\mathcal{H})$. The verticial morphisms are precisely the morphisms with nuclear domain which are, moreover, minimal-adjoint [cf. §0] to the morphisms with nuclear codomain. The vertices of the underlying semigraph of an object $\mathcal{H}$ are precisely the isomorphism classes, over $\mathcal{H}$, of verticial morphisms $\mathcal{K} \rightarrow \mathcal{H}$. Thus, in particular, we conclude that $\Phi$ induces a bijection between the sets of vertices of the underlying semi-graphs of corresponding objects, together with compatible isomorphisms of the various constituent anabelioids at the vertices; moreover, these bijections and isomorphisms are compatible with arrows in $\mathcal{C}$. In particular, $\Phi$ preserves locally trivial morphisms.

An object $\mathcal{H}$ is a link if and only if $\mathcal{H}$ is of verticial length 2, and, moreover, any locally trivial morphism $\mathcal{K} \rightarrow \mathcal{H}$, where $\mathcal{K}$ is also of verticial length 2 , is an isomorphism. The closed edges of the underlying semigraph of an object $\mathcal{H}$ are precisely the isomorphism classes, over $\mathcal{H}$, of locally trivial morphisms $\mathcal{K} \rightarrow \mathcal{H}$, where $\mathcal{K}$ is a link. An [open] edge of a nuclear object $\mathcal{H}$ is a system of compatible closed edges of NL-morphisms $\mathcal{H} \rightarrow \mathcal{K}$, as we vary the NL-morphism as described in Remark 4.7.1. Thus, we conclude that $\Phi$ induces an isomorphism between the underlying semi-graphs of corresponding objects; moreover, these isomorphisms are compatible with arrows in $\mathcal{C}$. Note that this implies, for instance, that $\Phi$ preserves the embeddings.

In particular, $\Phi$ preserves the finite objects [i.e., objects of finite verticial length], as well as the $N L$-morphisms. Moreover, by Proposition 4.6, $\Phi$ preserves the finite étale morphisms between finite objects. Thus, by considering the isotropy subgroups associated to the various vertices and edges in the Galois group of a finite étale Galois covering, one sees that $\Phi$ induces an isomorphism between the underlying semi-graphs of anabelioids of corresponding finite objects in a fashion that is compatible with arrows in $\mathcal{C}$. Moreover, these induced isomorphisms may be extended immediately to the case of infinite objects [i.e., when such objects exist in $\mathcal{C}$ ] by representing such objects as inductive limits of inductive systems consisting of finite objects and embeddings. 
Next, we observe that the closed objects $\mathcal{H}$ of $\mathcal{C}$ are precisely the finite objects whose underlying semi-graph $\mathbb{H}$ is a graph; $\mathcal{G}_{i}$ is the unique closed object of $\mathcal{C}$, up to isomorphism, to which every other closed object maps.

Next, we observe that one may recover the various local $(\mathcal{G}, \Gamma)$-structures on open objects as follows: First, we note that we may reconstruct the automorphisms $\mathcal{G}_{i} \rightarrow \mathcal{G}_{i}$ in $\Gamma_{i}$ by localizing on $\mathcal{G}_{i}$. That is to say, if $\phi: \mathcal{H} \rightarrow \mathcal{G}_{i}$ is a morphism with finite open domain [where we note that such a $\phi$ always exists, for instance, if $\mathbb{H}$ is a tree - cf. Proposition 4.3, (iv)], then by Proposition $4.3,(\mathrm{v})$, the $\Gamma_{i}$-span of $\phi$ is precisely the set of all morphisms $\mathcal{H} \rightarrow \mathcal{G}_{i}$ in $\mathcal{C}$. Moreover, since $\Gamma_{i}$ acts piecewise faithfully, it follows that the cardinality of this set is always equal to the order of $\Gamma_{i}$. Thus, by taking $\mathcal{H}$ to be various localizations of $\mathcal{G}_{i}$ and then gluing, we recover first the set of morphisms of semi-graphs of anabelioids $\mathcal{G}_{i} \rightarrow \mathcal{G}_{i}$ arising from elements of $\Gamma_{i}$ and then the group structure [by composing morphisms of semi-graphs of anabelioids].

Thus, in summary, we have shown that $\Phi$ induces an isomorphism of semigraphs of anabelioids

$$
\mathcal{G}_{1} \stackrel{\sim}{\rightarrow} \mathcal{G}_{2}
$$

together with a compatible isomorphism $\Gamma_{1} \stackrel{\sim}{\rightarrow} \Gamma_{2}$. Moreover, we have shown that $\Phi$ induces an isomorphism between the underlying semi-graph of anabelioids of corresponding objects in a fashion that is compatible with arrows in $\mathcal{C}$, as well as with the given local $(\mathcal{G}, \Gamma)$-structures. Thus, it is an easily verified tautology that the equivalence $\Phi$ is isomorphic to the equivalence induced by the isomorphisms $\mathcal{G}_{1} \stackrel{\sim}{\rightarrow} \mathcal{G}_{2}, \Gamma_{1} \stackrel{\sim}{\rightarrow} \Gamma_{2}$.

Finally, it remains to verify that $\mathcal{C}$ is $\operatorname{slim}$. Let $A \in \mathrm{Ob}(\mathcal{C})$; suppose that $\psi$ is an automorphism of the natural functor $\mathcal{C}_{A} \rightarrow \mathcal{C}$. Concretely speaking, this means that for every object $\beta: B \rightarrow A$ of $\mathcal{C}_{A}$, we are given an automorphism $\psi_{\beta} \in \operatorname{Aut}_{\mathcal{C}}(B)$ in such a way that the assignment $\beta \mapsto \psi_{\beta}$ is compatible with the image in $\mathcal{C}$ of arrows of $\mathcal{C}_{A}$. Since $A$ is arbitrary, it suffices [by considering, for given $\beta: B \rightarrow A$, the automorphism of the resulting composite functor $\mathcal{C}_{B} \rightarrow \mathcal{C}_{A} \rightarrow \mathcal{C}$ induced by $\psi$ ] to show that $\psi_{A}$ [i.e., the automorphism assigned to the identity $A \rightarrow A]$ is the identity. By considering $\beta$ with nuclear domain, we conclude immediately that $\psi_{\beta}$ fixes the set of vertices of $A$. Thus, since $\mathcal{G}_{i}$ is totally aloof, we reduce to the case where $A$ is nuclear.

Now since $A$ is nuclear, to show that $\psi$ is trivial, it suffices to show the following: If $v$ be a vertex of $\mathbb{G}_{i} ; \Gamma_{v} \subseteq \Gamma_{i}$ is the corresponding isotropy subgroup; and $H_{v}$ is the extension of $\Gamma_{v}$ by $\widehat{\pi}_{1}\left(\left(\mathcal{G}_{i}\right)_{v}\right)$ arising from the action of $\Gamma_{v}$ on $\left(\mathbb{G}_{i}\right)_{v}$, then the profinite group $H_{v}$ is slim. But this is an immediate formal 
consequence of the fact that $\mathcal{G}_{i}$ is verticially slim and totally aloof, together with our assumption that the action of $\Gamma_{i}$ on $\mathcal{G}_{i}$ is piecewise faithful.

Remark 4.8.1. Returning to the notation used in the discussion preceding Theorem 4.8, suppose that the semi-graph $\mathbb{G}$ has at least one vertex. Write $\mathbb{H} \subseteq \mathbb{G}$ for the maximal subgraph [cf. $\S 1$ ] of $\mathbb{G}$. Then observe that [whenever $\mathfrak{L o c}(\mathcal{G}, \Gamma)$ is defined] if we set $\mathcal{H} \stackrel{\text { def }}{=} \mathcal{G}_{\mathbb{H}}$, then $\Gamma$ acts naturally and piecewise faithfully on $\mathcal{H} ; \mathfrak{L o c}(\mathcal{H}, \Gamma)$ is defined; and we have natural equivalences

$$
\mathfrak{L o c}(\mathcal{G}, \Gamma)^{\mathrm{fin}} \stackrel{\sim}{\rightarrow} \mathfrak{L o c}(\mathcal{H}, \Gamma)^{\mathrm{fin}} ; \quad \mathfrak{L o c}(\mathcal{G}, \Gamma) \stackrel{\sim}{\rightarrow} \mathfrak{L o c}(\mathcal{H}, \Gamma)
$$

[defined by simply omitting all $\mathbb{G}$-open edges]. Thus, there is no essential loss of generality in restricting Theorem 4.8 to the case where $\mathbb{G}$ is a graph.

Remark 4.8.2. One verifies easily that [whenever $\mathfrak{L o c}(\mathcal{G}, \Gamma)$ is defined] the following five conditions are equivalent:

(i) $\mathbb{G}$ has no closed edges.

(ii) $\mathbb{G}$ is a tree [cf. $\S 1]$ with at most one vertex.

(iii) $\mathfrak{L o c}(\mathcal{G}, \Gamma)^{\perp}$ is a Galois category.

(iv) Every monomorphism of $\mathfrak{L o c}(\mathcal{G}, \Gamma)$ is an isomorphism.

(v) Every object of $\mathfrak{L o c}(\mathcal{G}, \Gamma)$ is closed.

Moreover, if any of these conditions is satisfied, then there is a natural equivalence

$$
\mathfrak{L o c}(\mathcal{G}, \Gamma)^{\perp} \stackrel{\sim}{\rightarrow} \mathcal{B}(\mathcal{G})
$$

— so, in particular, $\mathfrak{L o c}(\mathcal{G}, \Gamma)^{\perp}$ does not depend on the action of $\Gamma$ [which is, at any rate, trivial, if $\mathbb{G}$ admits at least one vertex]. Thus, since equivalences between connected anabelioids are "well-understood", there is no essential loss of generality in excluding from Theorem 4.8 the case in which these conditions are satisfied.

Remark 4.8.3. In the resp'd case of Theorem 4.8 [i.e., where one includes the infinite objects], if one assumes further that $\mathcal{G}_{i}$ is totally estranged, then the proof of Theorem 4.8 may be simplified somewhat, by applying Proposition 4.5, Corollary 3.9. 
Remark 4.8.4. In the notation of the proof of Theorem 4.8, if an object $A$ of $\mathcal{C}$ is nuclear, then the category $\mathcal{C}_{A}^{\perp}$ is easily verified to be a connected anabelioid; the category $\mathcal{C}[A]^{\perp}$ [cf. $\left.\S 0\right]$ is easily verified to be a "connected quasi-anabelioid" [cf. Remark A.4.2 of the Appendix].

\section{§5. Arithmetic Semi-graphs of Anabelioids}

In this $\S$, we consider semi-graphs of anabelioids equipped with a continuous action of a profinite group, which we think of as an "arithmetic structure" on the semi-graph of anabelioids. We then proceed to study a certain "arithmetic analogue" of the theory of maximal compact subgroups of $\S 3$.

Definition 5.1. (i) Let $\mathcal{G}$ be a connected, coherent, totally aloof, verticially slim semi-graph of anabelioids. Let $\mathcal{A}$ be a slim connected anabelioid, equipped with a basepoint, so we may speak of $\widehat{\pi}_{1}(\mathcal{A})$. We shall refer to as an action of $\widehat{\pi}_{1}(\mathcal{A})$ on $\mathcal{G}$ the datum of a homomorphism

$$
\rho_{\mathcal{G}}: \widehat{\pi}_{1}(\mathcal{A}) \rightarrow \operatorname{Aut}(\mathcal{G})
$$

[where $\operatorname{Aut}(\mathcal{G})$ denotes the group of automorphisms of $\mathcal{G}$ as a totally aloof, verticially slim semi-graph of anabelioids]. Note that any such pair $\left(\mathcal{G}, \rho_{\mathcal{G}}\right)$ admits an "inner action" by $\widehat{\pi}_{1}(\mathcal{A})$ - i.e., by letting $\widehat{\pi}_{1}(\mathcal{A})$ act on $\widehat{\pi}_{1}(\mathcal{A})$ by conjugation and on $\mathcal{G}$ via $\rho_{\mathcal{G}}$. We shall say that an action of $\widehat{\pi}_{1}(\mathcal{A})$ on $\mathcal{G}$ is continuous if, for some open subgroup $H \subseteq \widehat{\pi}_{1}(\mathcal{A})$, the following conditions are satisfied:

(a) $\widehat{\pi}_{1}(\mathcal{A})$ is topologically finitely generated.

(b) The semi-graph $\mathbb{G}$ is locally finite.

(c) The action of $H$ on $\mathbb{G}$ is trivial; the resulting outer homomorphism $H \rightarrow$ $\operatorname{Out}\left(\widehat{\pi}_{1}\left(\mathcal{G}_{v}\right)\right)$, where $v$ ranges over the vertices of $\mathbb{G}$, is continuous [i.e., relative to the natural profinite group topology on $\left.\operatorname{Out}\left(\widehat{\pi}_{1}\left(\mathcal{G}_{v}\right)\right)\right]$.

(d) There is a finite set $V$ of vertices of $\mathbb{G}$ such that for every vertex $w$ of $\mathbb{G}$, there exists a $v \in V$ and an isomorphism of semi-graphs of anabelioids $\mathcal{G}[v] \stackrel{\sim}{\rightarrow} \mathcal{G}[w]$ that is compatible with the action of $H$ on both sides.

(ii) A triple $\overline{\mathcal{G}}=\left(\mathcal{G}, \mathcal{A}, \rho_{\mathcal{G}}\right)$ as in (i), where $\rho_{\mathcal{G}}$ is a continuous action of $\widehat{\pi}_{1}(\mathcal{A})$ on $\mathcal{G}$, will be referred to as a connected arithmetic semi-graph of anabelioids (over $\mathcal{A})$. Suppose that $\overline{\mathcal{G}}=\left(\mathcal{G}, \mathcal{A}, \rho_{\mathcal{G}}\right)$ is a connected arithmetic semi-graph 
of anabelioids. Then we shall refer to $\mathcal{G}$ (respectively, $\mathcal{A} ; \rho_{\mathcal{G}}$ ) as the geometric component (respectively, arithmetic component; arithmetic action) of $\overline{\mathcal{G}}$. The arithmetic action of an arithmetic semi-graph of groups induces [what, by abuse of terminology, we shall also refer to as] "arithmetic actions" on various objects [e.g., the underlying semi-graph, etc.] associated to this arithmetic semi-graph of groups.

(iii) A [not necessarily connected] arithmetic semi-graph of anabelioids $\overline{\mathcal{G}}$ is defined to be a formal collection of connected arithmetic semi-graphs of anabelioids; each object in this collection will be referred to as a connected component of $\overline{\mathcal{G}}$. Note that the geometric components of each of the connected components of $\overline{\mathcal{G}}$ together determine a natural geometric component [i.e., a (not necessarily connected) semi-graph of anabelioids] of $\overline{\mathcal{G}}$. We shall say that an arithmetic semi-graph of anabelioids is finite (respectively, elevated at a vertex; totally elevated; sub-coverticial at a closed edge; universally sub-coverticial at a closed edge; totally sub-coverticial; totally universally sub-coverticial; aloof at an edge; totally aloof; estranged at an edge; totally estranged) if its geometric component is so.

(iv) Given two connected arithmetic semi-graphs of anabelioids $\overline{\mathcal{G}}^{\prime}=$ $\left(\mathcal{G}^{\prime}, \mathcal{A}^{\prime}, \rho_{\mathcal{G}^{\prime}}\right), \overline{\mathcal{G}}=\left(\mathcal{G}, \mathcal{A}, \rho_{\mathcal{G}}\right)$, a morphism of connected arithmetic semi-graphs of anabelioids

$$
\overline{\mathcal{G}}^{\prime} \rightarrow \overline{\mathcal{G}}
$$

consists of a pair

$$
\widehat{\pi}_{1}\left(\mathcal{A}^{\prime}\right) \rightarrow \widehat{\pi}_{1}(\mathcal{A}) ; \quad \mathcal{G}^{\prime} \rightarrow \mathcal{G}
$$

[i.e., a continuous homomorphism of profinite groups and a morphism of semigraphs of anabelioids] which is compatible with $\rho_{\mathcal{G}^{\prime}}, \rho_{\mathcal{G}}$, and which we regard up to composition with the inner action of $\widehat{\pi}_{1}(\mathcal{A})$ on $\left(\mathcal{G}, \rho_{\mathcal{G}}\right)$. We shall refer to the morphism $\mathcal{G}^{\prime} \rightarrow \mathcal{G}$ (respectively, the induced morphism of anabelioids $\mathcal{A}^{\prime} \rightarrow \mathcal{A}$ ) as the geometric (respectively, arithmetic) component of the morphism. [In particular, if we restrict our attention to $\overline{\mathcal{G}}^{\prime} \rightarrow \overline{\mathcal{G}}$ whose geometric component is locally open, then we may work with such morphisms as if they are "morphisms in a category" - cf. Remark 2.4.2.] A morphism of [not necessarily connected] arithmetic semi-graphs of anabelioids

$$
\overline{\mathcal{G}}^{\prime} \rightarrow \overline{\mathcal{G}}
$$

is defined to be a collection of morphisms, one from each connected component of $\overline{\mathcal{G}}^{\prime}$ to some connected component of $\overline{\mathcal{G}}$. We shall say that $\overline{\mathcal{G}}^{\prime} \rightarrow \overline{\mathcal{G}}$ 
is finite étale (respectively, tempered; locally trivial; locally open; locally finite étale; immersive; excisive; an embedding; BC-finite étale) if each of its geometric components is finite étale (respectively, a tempered covering [a term which we recall only makes sense when $\mathcal{G}$ is countable]; locally trivial; locally open; locally finite étale; immersive; excisive; an embedding; an isomorphism); each of its arithmetic components is finite étale (respectively, finite étale; an isomorphism; a composite of a $\pi_{1}$-epimorphism with a finite étale morphism; finite étale; an isomorphism; an isomorphism; an isomorphism; finite étale); and its induced map on connected components has finite [but possibly empty] fibers (respectively, has countable fibers; is arbitrary; is arbitrary; is arbitrary; is arbitrary; is arbitrary; is injective; has finite [but possibly empty] fibers). [Here, the abbreviation "BC" is to be understood to stand for the phrase "base of constants".]

Proposition 5.2 (Arithmetic Tempered Coverings). Let $\overline{\mathcal{G}}=\left(\mathcal{G}, \mathcal{A}, \rho_{\mathcal{G}}\right)$ be a connected arithmetic semi-graph of anabelioids. Then:

(i) Every tempered covering of $\mathcal{G}$ appears as the geometric component of a tempered covering of $\overline{\mathcal{G}}$.

(ii) Suppose that $\overline{\mathcal{H}} \rightarrow \overline{\mathcal{G}}, \overline{\mathcal{K}} \rightarrow \overline{\mathcal{G}}$ are tempered coverings with isomorphic geometric components. Then there exist BC-finite étale coverings $\overline{\mathcal{H}}^{\prime} \rightarrow \overline{\mathcal{H}}$, $\overline{\mathcal{K}}^{\prime} \rightarrow \overline{\mathcal{K}}$ such that $\overline{\mathcal{H}}^{\prime}, \overline{\mathcal{K}}^{\prime}$ are isomorphic as tempered coverings over $\overline{\mathcal{G}}$.

(iii) Let us denote by

$$
\mathcal{B}^{\text {temp }}(\overline{\mathcal{G}})
$$

the category whose objects are tempered morphisms $\overline{\mathcal{G}}^{\prime} \rightarrow \overline{\mathcal{G}}$ and whose morphisms are tempered morphisms over $\overline{\mathcal{G}}$. Then $\mathcal{B}^{\text {temp }}(\overline{\mathcal{G}})$ is a connected temperoid. Similarly, the full subcategory

$$
\mathcal{B}(\overline{\mathcal{G}}) \subseteq \mathcal{B}^{\text {temp }}(\overline{\mathcal{G}})
$$

determined by the finite étale coverings forms a connected anabelioid. If $\overline{\mathcal{G}}$ is totally elevated, then $\mathcal{B}^{\text {temp }}(\overline{\mathcal{G}})$ temp-slim, and $\mathcal{B}(\overline{\mathcal{G}})$ is slim.

(iv) Write:

$$
\begin{aligned}
\Pi_{\overline{\mathcal{G}}}^{\text {temp }} \stackrel{\text { def }}{=} \pi_{1}^{\text {temp }}(\overline{\mathcal{G}}) \stackrel{\text { def }}{=} \pi_{1}^{\text {temp }}\left(\mathcal{B}^{\text {temp }}(\overline{\mathcal{G}})\right) ; \quad \Pi_{\mathcal{G}}^{\text {temp }} \stackrel{\text { def }}{=} \pi_{1}^{\text {temp }}\left(\mathcal{B}^{\text {temp }}(\mathcal{G})\right)=\pi_{1}^{\text {temp }}(\mathcal{G}) \\
\Pi_{\overline{\mathcal{G}}} \stackrel{\text { def }}{=} \widehat{\pi}_{1}(\overline{\mathcal{G}}) \stackrel{\text { def }}{=} \widehat{\pi}_{1}(\mathcal{B}(\overline{\mathcal{G}})) ; \quad \Pi_{\mathcal{G}} \stackrel{\text { def }}{=} \widehat{\pi}_{1}(\mathcal{B}(\mathcal{G}))=\widehat{\pi}_{1}(\mathcal{G})
\end{aligned}
$$


Then there are natural morphisms

$$
\mathcal{B}^{\text {temp }}(\mathcal{G}) \rightarrow \mathcal{B}^{\text {temp }}(\overline{\mathcal{G}}) \rightarrow \mathcal{A}^{\top} ; \quad \mathcal{B}(\mathcal{G}) \rightarrow \mathcal{B}(\overline{\mathcal{G}}) \rightarrow \mathcal{A}
$$

which induce natural exact sequences:

$$
\begin{aligned}
1 \rightarrow \Pi_{\mathcal{G}}^{\text {temp }} & \rightarrow \Pi_{\overline{\mathcal{G}}}^{\text {temp }} \rightarrow \Pi_{\mathcal{A}} \rightarrow 1 \\
1 \rightarrow \Pi_{\mathcal{G}} & \rightarrow \Pi_{\overline{\mathcal{G}}} \rightarrow \Pi_{\mathcal{A}} \rightarrow 1
\end{aligned}
$$

We shall refer to $\Pi_{\mathcal{G}}$ (respectively, $\left.\Pi_{\mathcal{G}}^{\text {temp }} ; \Pi_{\overline{\mathcal{G}}} ; \Pi_{\overline{\mathcal{G}}}^{\text {temp }} ; \Pi_{\mathcal{A}} \stackrel{\text { def }}{=} \widehat{\pi}_{1}(\mathcal{A})\right)$ as the geometric (respectively, geometric tempered; arithmetic; arithmetic tempered; BC-) fundamental group of $\overline{\mathcal{G}}$.

Proof. Assertions (i), (ii) follow from the various finiteness assumptions in our definition of a "continuous action" [cf. Definition 5.1, (i); the fact that $\mathcal{G}$ is coherent]. [Note, in particular, that one must make use of the assumption of Definition 5.1, (i), (d), in order to verify assertions (i), (ii) for arbitrary infinite $\overline{\mathcal{G}}$.] Except for the final sentence of assertion (iii), assertions (iii), (iv) follow immediately from the definitions and assertions (i), (ii); the final sentence of assertion (iii) follows from Corollary 2.7, (ii).

Remark 5.2.1. At this point, one could proceed to develop a theory of "categories of arithmetic localizations" of arithmetic semi-graphs of anabelioids, in the style of $\S 4$. Although this is quite possible [we leave the details to the enthusiastic reader!], it is rather cumbersome, so instead we restrict ourselves [cf. Remark 4.8.3] to considering the categorical representation of arithmetic semi-graphs of anabelioids afforded by the "arithmetically maximal compact subgroups" [cf. Definition 5.3 below] of the tempered fundamental groups of Proposition 5.2, in the style of Corollary 3.9.

Let $\overline{\mathcal{G}}$ be a connected, countable, totally elevated, totally estranged arithmetic semi-graph of anabelioids, with underlying semi-graph $\mathbb{G}$. In the notation of Proposition 5.2, we would like to consider compact subgroups of the arithmetic tempered fundamental group $\Pi_{\overline{\mathcal{G}}}^{\text {temp }}$. Note that for every vertex $v$ of $\mathbb{G}$, we obtain an associated decomposition group

$$
\Pi_{\overline{\mathcal{G}}, v}^{\mathrm{temp}} \subseteq \Pi_{\overline{\mathcal{G}}}^{\mathrm{temp}}
$$

[well-defined up to conjugation in $\Pi_{\overline{\mathcal{G}}}^{\text {temp }}$ ], which [by Corollary 2.7 , (i), (iii); the injection of Proposition 3.6, (iii)] may be thought of as the commensurator in 
$\Pi_{\overline{\mathcal{G}}}^{\text {temp }}$ of $\Pi_{\mathcal{G}, v}^{\text {temp }} \stackrel{\text { def }}{=} \Pi_{\overline{\mathcal{G}}, v}^{\text {temp }} \cap \Pi_{\mathcal{G}}^{\text {temp }}$. Similarly, if $b$ is a branch of an edge $e$ of $\mathbb{G}$ that abuts to $v$, then we obtain a decomposition group

$$
\Pi_{\overline{\mathcal{G}}, b}^{\mathrm{temp}} \subseteq \Pi_{\overline{\mathcal{G}}, v}^{\mathrm{temp}} \subseteq \Pi_{\overline{\mathcal{G}}}^{\mathrm{temp}}
$$

[well-defined up to conjugation in $\Pi_{\overline{\mathcal{G}}}^{\text {temp }}$ ], which [since $\mathcal{G}$ is totally estranged, hence, in particular totally aloof] may be thought of as the commensurator in $\Pi_{\overline{\mathcal{G}}, v}^{\text {temp }}$ of $\Pi_{\mathcal{G}, b}^{\text {temp }} \stackrel{\text { def }}{=} \Pi_{\overline{\mathcal{G}}, b}^{\text {temp }} \cap \Pi_{\mathcal{G}}^{\text {temp }}$.

Definition 5.3. $\quad$ (i) A closed subgroup of $\Pi_{\overline{\mathcal{G}}}^{\text {temp }}$ will be called arithmetically ample if it surjects onto an open subgroup of $\Pi_{\mathcal{A}}$. A compact subgroup of $\Pi_{\overline{\mathcal{G}}}^{\text {temp }}$ will be called arithmetically maximal if it is maximal among arithmetically ample compact subgroups of $\Pi_{\overline{\mathcal{G}}}^{\mathrm{temp}}$.

(ii) Let $e$ be an edge of $\mathbb{G}$. We shall say that $e$ is arithmetically estranged if, for every vertex $v$ to which some branch $b$ of $e$ abuts and every $g \in \Pi_{\overline{\mathcal{G}}, v}^{\text {temp }}$, the intersection in $\Pi_{\overline{\mathcal{G}}, v}^{\text {temp }}$ of $\Pi_{\overline{\mathcal{G}}, b}^{\text {temp }}$ with any subgroup of the form $g \cdot \Pi_{\overline{\mathcal{G}}, b^{\prime}}^{\text {temp }} \cdot g^{-1}$, where either $b^{\prime} \neq b$ is a branch of an edge that abuts to $v$ or $b^{\prime}=b$ and $g \notin \Pi_{\overline{\mathcal{G}}, b}^{\mathrm{temp}}$, fails to be arithmetically ample. If every edge of $\mathbb{G}$ is arithmetically estranged, then we shall say that $\overline{\mathcal{G}}$ is totally arithmetically estranged.

(iii) We shall refer to subgroups of $\Pi_{\overline{\mathcal{G}}}^{\text {temp }}$ of the form " $\Pi_{\overline{\mathcal{G}}, v}^{\mathrm{temp}}$ " (respectively, " $\left.\Pi_{\overline{\mathcal{G}}, b}^{\mathrm{temp}} "\right)$ as verticial (respectively, edge-like).

Remark 5.3.1. $\quad$ Note that all verticial and edge-like subgroups of $\Pi_{\overline{\mathcal{G}}}^{\text {temp }}$ are compact and arithmetically ample. Also, the intersection with $\Pi_{\mathcal{G}}^{\text {temp }}$ of a(n) verticial (respectively, edge-like) subgroup of $\Pi_{\overline{\mathcal{G}}}^{\text {temp }}$ is a(n) verticial (respectively, edge-like) subgroup of $\Pi_{\mathcal{G}}^{\text {temp }}$ in the sense of Theorem 3.7.

The main result of the present $\S 5$ is the following "arithmetic analogue" of Theorem 3.7, Corollary 3.9:

Theorem 5.4 (Arithmetically Maximal Compact Subgroups). Let $\overline{\mathcal{G}}$, $\overline{\mathcal{H}}$ be connected, countable, totally elevated, totally arithmetically estranged arithmetic graphs of anabelioids, with the same arithmetic component $\mathcal{A}$. Suppose, moreover, that the arithmetic actions on the underlying graphs $\mathbb{G}, \mathbb{H}$ do not switch the branches of any edge. Then: 
(i) Every arithmetically ample compact subgroup of $\pi_{1}^{\text {temp }}(\overline{\mathcal{G}})$ is contained in at least one verticial subgroup. If an arithmetically ample compact subgroup of $\pi_{1}^{\text {temp }}(\overline{\mathcal{G}})$ is contained in more than one verticial subgroup, then it is contained in precisely two verticial subgroups, whose intersection forms an edge-like subgroup.

(ii) The arithmetically maximal compact subgroups of $\pi_{1}^{\text {temp }}(\overline{\mathcal{G}})$ are precisely the verticial subgroups. The arithmetically ample intersections of two distinct arithmetically maximal compact subgroups of $\pi_{1}^{\text {temp }}(\overline{\mathcal{G}})$ are precisely the edge-like subgroups.

(iii) Applying " $\mathcal{B}^{\text {temp }}(-)$ " determines a natural bijective correspondence between locally open morphisms of arithmetic semi-graphs of anabelioids

$$
\overline{\mathcal{G}} \rightarrow \overline{\mathcal{H}}
$$

over $\mathcal{A}$ and "arithmetically quasi-geometric" morphisms of temperoids $\mathcal{B}^{\text {temp }}(\overline{\mathcal{G}}) \rightarrow \mathcal{B}^{\text {temp }}(\overline{\mathcal{H}})$ over $\mathcal{A}^{\top}$, i.e., morphisms that arise from a continuous morphism $\Pi_{\overline{\mathcal{G}}}^{\text {temp }} \rightarrow \Pi_{\overline{\mathcal{H}}}^{\text {temp }}$ that maps any arithmetically maximal compact subgroup $K_{1} \subseteq \Pi_{\overline{\mathcal{G}}}^{\text {temp }}$ (respectively, arithmetically ample intersection $K_{1} \cap H_{1}$ of two distinct arithmetically maximal compact subgroups $K_{1}, H_{1} \subseteq \Pi_{\overline{\mathcal{G}}}^{\mathrm{temp}}$ ) to an open subgroup of some arithmetically maximal compact subgroup $K_{2} \subseteq \Pi_{\overline{\mathcal{H}}}^{\text {temp }}$ (respectively, of some arithmetically ample intersection $K_{2} \bigcap H_{2}$ of two distinct arithmetically maximal compact subgroups $K_{2}, H_{2} \subseteq \Pi_{\overline{\mathcal{H}}}^{\text {temp }}$ ).

Proof. Modulo the evident "arithmetic translation" — e.g., "nontrivial" is to be replaced by "arithmetically ample" and "estranged" by "arithmetically estranged" - the proofs are entirely parallel to those of Theorem 3.7, Corollary 3.9.

Before proceeding, we review the following well-known result:

Lemma 5.5 (Decomposition Groups of Proper Hyperbolic Curves over Finite Fields). Let $X$ be a proper hyperbolic curve over a finite field $k$. Write $\Pi_{X}$ for the étale fundamental group of $X ; \Pi_{X} \rightarrow G_{k}$ for the natural augmentation to the absolute Galois group of $k$. Then a $k$-valued point $x \in X(k)$ is determined by the outer homomorphism $\sigma_{x}: G_{k} \rightarrow \Pi_{X}$ that it induces.

Proof. Write $J$ for the Jacobian of $X$; assume for simplicity that there exists a point $x_{0} \in X(k)$. Then $x_{0}$ determines a closed embedding $X \hookrightarrow$ 
$J$ whose induced morphism on étale fundamental groups $\Pi_{X} \rightarrow \Pi_{J}$ may be identified with the quotient of $\Pi_{X}$ by the commutator subgroup of the kernel of the surjection $\Pi_{X} \rightarrow G_{k}$. Thus, it suffices to show that a point $a \in J(k)$ is determined by the outer homomorphism $\sigma_{a}: G_{k} \rightarrow \Pi_{J}$ that it induces.

Write $\sigma_{0}: G_{k} \rightarrow \Pi_{J}$ for the outer homomorphism induced by the identity element of $J(k)$. Then the difference between $\sigma_{a}$ and $\sigma_{0}$ may be thought of as an element of $\eta_{a} \in H^{1}(k, T)$, where we define $T$ to be the kernel of the natural surjection $\Pi_{J} \rightarrow G_{k}$. Note, moreover, that we have a natural isomorphism $T \stackrel{\sim}{\rightarrow} \operatorname{Hom}(\mathbb{Q} / \mathbb{Z}, J(\bar{k}))$, where $\bar{k}$ is the algebraic closure of $k$ determined by the basepoint of $k$ implicit in the discussion. On the other hand, by wellknown general nonsense [cf., e.g., [Naka], Claim (2.2); [NTs], Lemma (4.14); [Mzk2], the Remark preceding Definition 6.2], there is a natural isomorphism $H^{1}(k, T) \stackrel{\sim}{\rightarrow} J(k)$, which maps $\eta_{a}$ to $a$. In particular, $\eta_{a}$, hence also $\sigma_{a}$, is sufficient to determine $a$ itself.

\section{Example 5.6. Pointed Stable Curves over $p$-adic Local Fields II.}

We work in the notation of Example 3.10. Also, Suppose that we are given an exhaustive sequence of open characteristic [hence normal] subgroups of finite index

$$
\cdots \subseteq M_{i} \subseteq \cdots \subseteq \Pi
$$

[where $i$ ranges over the positive integers] of $\Pi$ such that $N_{i}=M_{i} \cap \Delta$; write $\Pi_{i} \stackrel{\text { def }}{=} \Pi / M_{i}$. Thus, $M_{i}$ determines a finite log étale covering of $X_{K}^{\log }$; we assume that $M_{i}$ has been chosen so that this covering has stable reduction over the ring of integers of the finite extension of $K$ that it determines. Then the outer action of $M_{i}$ on $N_{i}$ determines an arithmetic action on the semi-graphs of anabelioids $\mathcal{G}_{i}, \mathcal{G}_{i}^{\mathrm{c}}$ of Example 3.10; that is to say, we obtain arithmetic semi-graphs of anabelioids

$$
\overline{\mathcal{G}}_{i} ; \quad \overline{\mathcal{G}}_{i}^{\mathrm{c}}
$$

with underlying semi-graphs of anabelioids $\mathcal{G}_{i}, \mathcal{G}_{i}^{\text {c }}$, respectively, equipped with natural actions by $\Pi_{i}$. Moreover, $\overline{\mathcal{G}}_{i}, \overline{\mathcal{G}}_{i}^{\mathrm{c}}$ are connected, finite, totally elevated, and totally universally sub-coverticial [cf. Example 3.10]. Also, it follows immediately from Lemma 5.5 that $\overline{\mathcal{G}}_{i}, \overline{\mathcal{G}}_{i}^{\mathrm{c}}$ are totally arithmetically estranged. In particular, [at least for $i$ sufficiently large] $\overline{\mathcal{G}}_{i}$ satisfies the hypotheses of Theorem 5.4.

Now I claim that the generalized morphisms of arithmetic graphs of anabelioids

$$
\overline{\mathcal{G}}_{i} \rightarrow \overline{\mathcal{G}}_{j}
$$


[where $i \geq j$ ] - i.e., the generalized morphisms of graphs of anabelioids of Example 3.10 considered together with the natural compatible arithmetic actions on the domain and codomain - may be recovered group-theoretically from the associated morphisms of tempered fundamental groups

$$
\Pi_{\overline{\mathcal{G}}_{i}}^{\text {temp }} \rightarrow \Pi_{\overline{\mathcal{G}}_{j}}^{\text {temp }}
$$

as follows: First, we consider the functor $\operatorname{Cat}\left(\mathbb{G}_{i}\right) \rightarrow \operatorname{Cat}\left(\mathbb{G}_{j}\right)$. Now observe that if $v$ (respectively, $e$ ) is a(n) vertex (respectively, edge) of $\mathbb{G}_{i}$ such that the image in $\Pi_{\overline{\mathcal{G}}_{j}}^{\text {temp }}$ of the verticial (respectively, edge-like) subgroup determined by $v$ (respectively, $e$ ) is contained in a [necessarily unique] edge-like subgroup $H$ of $\Pi_{\overline{\mathcal{G}}_{j}}^{\text {temp }}$, then this functor maps $v$ (respectively, e) to the edge of $\mathbb{G}_{j}$ determined by $H$. On the other hand, if $v$ (respectively, $e$ ) is a(n) vertex (respectively, edge) of $\mathbb{G}_{i}$ such that the image in $\Pi_{\overline{\mathcal{G}}_{j}}^{\text {temp }}$ of the verticial (respectively, edgelike) subgroup determined by $v$ (respectively, $e$ ) is not contained in an edge-like subgroup of $\Pi_{\overline{\mathcal{G}}_{j}}^{\text {temp }}$, but $i s$ contained in a verticial subgroup $H$ of $\Pi_{\overline{\mathcal{G}}_{j}}^{\text {temp }}$, then this functor maps $v$ (respectively, e) to the vertex of $\mathbb{G}_{j}$ determined by $H$ [cf. Lemma 5.5 in the case where this image fails to be an open subgroup of $H$ ]. That these characterizations make sense and, moreover, do indeed yield the map on objects determined by the functor in question follows from Theorem 5.4, (i), (ii); Lemma 5.5. The remainder of the data necessary to define the generalized morphism of arithmetic graphs of anabelioids $\overline{\mathcal{G}}_{i} \rightarrow \overline{\mathcal{G}}_{j}$ is determined naturally by considering the maps between the various verticial and edge-like subgroups of $\Pi_{\overline{\mathcal{G}}_{i}}^{\text {temp }}, \Pi_{\overline{\mathcal{G}}_{j}}^{\text {temp }}$. This completes the proof of the claim.

Moreover, by a similar argument, together with the technique of Corollary 3.11, one may reconstruct the generalized morphisms of arithmetic semi-graphs of anabelioids

$$
\overline{\mathcal{G}}_{i}^{\mathrm{c}} \rightarrow \overline{\mathcal{G}}_{j}^{\mathrm{c}}
$$

[where $i \geq j$ ] group-theoretically from the corresponding morphisms of tempered groups $M_{i} \rightarrow M_{j}$.

Remark 5.6.1. There is an immediate profinite generalization of the group-theoretic reconstruction in Example 5.6 of the generalized morphism of arithmetic graphs of anabelioids

$$
\overline{\mathcal{G}}_{i}^{\mathrm{c}} \rightarrow \overline{\mathcal{G}}_{j}^{\mathrm{c}}
$$

[where $i \geq j$ ] from the corresponding morphism of profinite groups:

$$
M_{i}^{\wedge} \rightarrow M_{j}^{\wedge}
$$


[where the " $\wedge$ " denotes profinite completion]. Indeed, this follows by applying [in place of Theorem 5.4, the technique of Corollary 3.11] the fact that the "dual semi-graph with compact structure of the geometric special fiber" may be recovered even in the profinite case, from the Galois action on the geometric profinite fundamental group [cf. [Mzk3], Lemma 2.3].

\section{§6. Tempered Anabelian Geometry}

In this $\S$, we observe that the theory of Galois sections in absolute anabelian geometry [cf. [Mzk8]] admits a fairly straightforward generalization to the case of tempered fundamental groups.

Let $K$ be a finite extension of $\mathbb{Q}_{p} ; \bar{K}$ an algebraic closure of $K ; X_{K}$ a hyperbolic curve over $K$. Let us write $X_{\bar{K}} \stackrel{\text { def }}{=} X_{K} \times_{K} \bar{K}$;

$$
\pi_{1}^{\mathrm{temp}}\left(X_{K}\right)
$$

for the tempered fundamental group of [André], $\S 4$ [cf. also the group " $\pi_{1}^{\text {temp }}\left(X_{K}^{\log }\right)$ " of Examples $\left.3.10,5.6\right]$. Thus, $\pi_{1}^{\text {temp }}\left(X_{K}\right)$ is a tempered topological group [in the sense of Definition 3.1, (i)] and fits into a natural exact sequence:

$$
1 \rightarrow \pi_{1}^{\mathrm{temp}}\left(X_{\bar{K}}\right) \rightarrow \pi_{1}^{\mathrm{temp}}\left(X_{K}\right) \rightarrow G_{K} \rightarrow 1
$$

[where $G_{K} \stackrel{\text { def }}{=} \operatorname{Gal}(\bar{K} / K)$; we write $\pi_{1}^{\text {temp }}\left(X_{\bar{K}}\right)$ for the geometric tempered fundamental group of $X_{K}$, i.e., the tempered fundamental group of $X_{\bar{K}} \times_{\bar{K}} \widehat{\bar{K}}$; the " $\wedge$ " denotes the $p$-adic completion]. To simplify the notation, let us write:

$$
\Pi_{X_{K}}^{\text {temp }} \stackrel{\text { def }}{=} \pi_{1}^{\text {temp }}\left(X_{K}\right) ; \quad \Delta_{X}^{\text {temp }} \stackrel{\text { def }}{=} \pi_{1}^{\text {temp }}\left(X_{\bar{K}}\right)
$$

In the following discussion, we shall denote the profinite completion of a group by means of a " $\wedge$ ". Also, we shall write $\Pi_{X_{K}} \stackrel{\text { def }}{=} \widehat{\Pi}_{X_{K}}^{\text {temp }} ; \Delta_{X} \stackrel{\text { def }}{=} \widehat{\Delta}_{X}^{\text {temp }}$. It follows from the well-known residual finiteness of discrete free groups [cf., e.g., Corollary 1.7] that we have natural injections $\Pi_{X_{K}}^{\text {temp }} \hookrightarrow \Pi_{X_{K}}, \Delta_{X}^{\text {temp }} \hookrightarrow \Delta_{X}$ [cf. the discussion of [André], §4.5].

Lemma 6.1 (Profinite Normalizers).

(i) Let $F$ be a finitely generated [discrete] free group of rank $>1$. Then $N_{\widehat{F}}(F)=F$.

(ii) We have: $N_{\Delta_{X}}\left(\Delta_{X}^{\text {temp }}\right)=\Delta_{X}^{\text {temp }}$. 
(iii) We have: $N_{\Pi_{X_{K}}}\left(\Pi_{X_{K}}^{\text {temp }}\right)=\Pi_{X_{K}}^{\text {temp }}$.

Proof. Assertion (i) (respectively, (ii)) is the content of [André], Lemma 3.2.1 (respectively, [André], Corollary 6.2.2). Assertion (iii) follows immediately from assertion (ii).

Definition 6.2. $\quad$ Let $F, F_{1}, F_{2}$ be tempered groups [cf. Definition 3.1, (i)]. Then:

(i) We shall refer to a [not necessarily closed] subgroup $H \subseteq F$ as being of DFG-type [i.e., "dense, finitely generated type"] if it is dense in some open subgroup of the profinite completion $\widehat{F}$, and, moreover, for any open normal subgroup $J \subseteq F$, the image of $H$ in $F / J$ is finitely generated.

(ii) We shall refer to a [not necessarily closed] subgroup $H \subseteq F$ as being of DOF-type [i.e., "dense in an open subgroup of finite index type"] if it is dense in some open subgroup of $F$ of finite index.

(iii) A continuous homomorphism $F_{1} \rightarrow F_{2}$ will be said to be of $D F G$ type (respectively, of DOF-type) if its image is a subgroup of $F_{2}$ of DFG-type (respectively, of DOF-type).

Lemma 6.3 (Dense Subgroups).

(i) Let $F$ be a finitely generated [discrete] free group of rank $>1$. Suppose that $H \subseteq F$ is a finitely generated subgroup which is dense in $\widehat{F}$. Then $H=F$.

(ii) Let $F$ be either $\Pi_{X_{K}}^{\text {temp }}$ or $\Delta_{X}^{\text {temp }}$; write $\widehat{F}$ for the profinite completion of $F$. Then a subgroup $H \subseteq F$ is of DFG-type if and only if it is of DOF-type.

(iii) Let $F, \widehat{F}$ be as in (ii). Suppose that $F_{1}, F_{2} \subseteq F$ are subgroups of DOFtype which are dense in $\widehat{F}$. Then, for any $f \in \widehat{F}$ such that $f \cdot F_{1} \cdot f^{-1}=F_{2}$, it follows that $f \in F$.

Proof. Assertion (i) follows immediately from the "structure theory of finitely generated subgroups of free groups of finite rank" [cf., e.g., Corollary 1.6, (ii)]. As for assertion (ii), let us first observe that by replacing $F$ by an open subgroup of $F$ of finite index containing $H$, we may assume that $H$ is 
dense in $\widehat{F}$. Now sufficiency is immediate. To prove necessity, we note that it follows from assertion (i), together with the assumption that $H$ is dense in $\widehat{F}$, that the image of $H$ in each $F / J$ is equal to $F / J$, i.e., that $H$ is dense in $F$, as desired. Finally, assertion (iii) follows from Lemma 6.1, (i) [cf. the proofs of Lemma 6.1, (ii), (iii)].

Now suppose that $L \subseteq \bar{K}$ is also a finite extension of $\mathbb{Q}_{p} ; Y_{L}$ is a hyperbolic curve over $L$. We shall use similar notation for the various fundamental groups [i.e., tempered, profinite étale, etc.] associated to $Y_{L}$ to the notation used thus far for $X_{K}$. Now we have the following result [cf. [Mzk8], Theorem 1.2]:

Theorem 6.4 (Tempered Anabelian Theorem for Hyperbolic Curves over Local Fields). The tempered fundamental group functor determines a bijection between the set of dominant morphisms of schemes

$$
X_{K} \rightarrow Y_{L}
$$

and the set of outer homomorphisms of DOF-type $\phi: \Pi_{X_{K}}^{\text {temp }} \rightarrow \Pi_{Y_{L}}^{\text {temp }}$ that fit into a commutative diagram

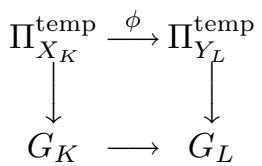

for which the induced morphism $G_{K} \rightarrow G_{L}$ is an open immersion [i.e., an isomorphism onto an open subgroup of $G_{L}$ ] which arises from an embedding of fields $L \hookrightarrow K$.

Proof. One verifies immediately from the definition of the "tempered fundamental group" that any $\Pi_{X_{K}}^{\text {temp }} \rightarrow \Pi_{Y_{L}}^{\text {temp }}$ that arises geometrically is of $D F G$-type, hence, by Lemma 6.3, (ii), of DOF-type. On the other hand, given a homomorphism $\phi: \Pi_{X_{K}}^{\text {temp }} \rightarrow \Pi_{Y_{L}}^{\text {temp }}$ of DOF-type, profinite completion yields an open homomorphism $\widehat{\phi}: \Pi_{X_{K}} \rightarrow \Pi_{Y_{L}}$, so by [Mzk8], Theorem 1.2 [i.e., in essence, [Mzk2], Theorem A], we obtain that $\widehat{\phi}$ arises, up to inner automorphism, from a dominant morphism of schemes $X_{K} \rightarrow Y_{L}$. In particular, this dominant morphism of schemes induces a homomorphism $\psi: \Pi_{X_{K}}^{\text {temp }} \rightarrow \Pi_{Y_{L}}^{\text {temp }}$ of DOF-type, whose profinite completion $\widehat{\psi}: \Pi_{X_{K}} \rightarrow \Pi_{Y_{L}}$ differs from $\widehat{\phi}$ by composition with an inner automorphism of $\Pi_{Y_{L}}$. On the other hand, by Lemma 6.3 , (iii), we thus conclude that $\phi$ differs from $\psi$ by composition with an inner automorphism of $\Pi_{Y_{L}}^{\text {temp }}$, as desired. 
Next, let us write $X_{K} \hookrightarrow \bar{X}_{K}$ for the compactification [cf. $\S 0$ ] of $X_{K}$. Let

$$
x \in \bar{X}_{K}
$$

be a closed point. Thus, $x$ determines, up to conjugation by an element of $\Pi_{X_{K}}^{\text {temp }}$, a decomposition group:

$$
D_{x} \subseteq \Pi_{X_{K}}^{\mathrm{temp}}
$$

We shall refer to a closed subgroup of $\Pi_{X_{K}}^{\mathrm{temp}}$ which arises in this way as a decomposition group of $\Pi_{X_{K}}^{\text {temp }}$. If $x$ is a cusp, then we shall refer to the decomposition group $D_{x}$ as cuspidal. Note that $D_{x}$ always surjects onto an open subgroup of $G_{K}$. Moreover, the subgroup

$$
I_{x} \stackrel{\text { def }}{=} D_{x} \bigcap \Delta_{X}^{\text {temp }}
$$

is isomorphic to $\widehat{\mathbb{Z}}(1)$ [i.e., the profinite completion of $\mathbb{Z}$, Tate twisted once] (respectively, $\{1\})$ if $x$ is (respectively, is not) a cusp. We shall refer to a closed subgroup of $\Pi_{X_{K}}^{\text {temp }}$ which is equal to " $I_{x}$ " for some cusp $x$ as a cuspidal geometric decomposition group.

Theorem 6.5 (Tempered Decomposition Groups).

(i) (Determination of the Point) The closed point $x$ is completely determined by the conjugacy class of the closed subgroup $D_{x} \subseteq \Pi_{X_{K}}^{\text {temp }}$. If $x$ is a cusp, then $x$ is completely determined by the conjugacy class of the closed subgroup $I_{x} \subseteq \Pi_{X_{K}}^{\text {temp }}$.

(ii) (Commensurable Terminality) The subgroup $D_{x}$ is commensurably terminal in $\Pi_{X_{K}}^{\text {temp }}$. If $x$ is a cusp, then $D_{x}=C_{\Pi_{X_{K}}^{\text {temp }}}(H)$ for any open subgroup $H \subseteq I_{x}$

(iii) (Absoluteness of Cuspidal Decomposition Groups) Every isomorphism of tempered groups

$$
\alpha: \Pi_{X_{K}}^{\mathrm{temp}} \stackrel{\sim}{\rightarrow} \Pi_{Y_{L}}^{\mathrm{temp}}
$$

preserves cuspidal decomposition groups and cuspidal geometric decomposition groups.

(iv) (Cuspidal and Noncuspidal Decomposition Groups) No noncuspidal decomposition group of $\Pi_{X_{K}}^{\text {temp }}$ is contained in a cuspidal decomposition group of $\Pi_{X_{K}}^{\text {temp }}$. 
Proof. Assertions (i), (ii), (iv) follow formally from [Mzk8], Theorem 1.3, (i), (ii), (iv), respectively. Assertion (iii) follows from Corollary 3.11.

To a large extent, the absolute anabelian geometry of tempered fundamental groups is essentially equivalent to the absolute anabelian geometry of profinite fundamental groups. Indeed, we have the following result:

Theorem 6.6 (Tempered and Profinite Outer Isomorphisms). Every outer isomorphism

$$
\Pi_{X_{K}} \stackrel{\sim}{\rightarrow} \Pi_{Y_{L}}
$$

of profinite groups arises from a unique outer isomorphism

$$
\Pi_{X_{K}}^{\mathrm{temp}} \stackrel{\sim}{\rightarrow} \Pi_{Y_{L}}^{\mathrm{temp}}
$$

of tempered groups.

Proof. Indeed, it is immediate that every outer isomorphism $\Pi_{X_{K}}^{\text {temp }}$ $\stackrel{\sim}{\rightarrow} \Pi_{Y_{L}}^{\text {temp }}$ determines an outer isomorphism $\Pi_{X_{K}} \stackrel{\sim}{\rightarrow} \Pi_{Y_{L}}$. Now let

$$
\widehat{\alpha}: \Pi_{X_{K}} \stackrel{\sim}{\rightarrow} \Pi_{Y_{L}}
$$

be an arbitrary outer isomorphism. Let $H_{X} \subseteq \Pi_{X_{K}}, H_{Y} \subseteq \Pi_{Y_{L}}$ be open normal subgroups [of finite index] that correspond via $\widehat{\alpha}$. Then by [Mzk3], Lemma 2.3, $\widehat{\alpha}$ determines a natural isomorphism

$$
\widehat{\alpha}_{H}: \mathcal{G}_{H_{X}}^{\mathrm{c}} \stackrel{\sim}{\rightarrow} \mathcal{G}_{H_{Y}}^{\mathrm{c}}
$$

between the "semi-graphs of anabelioids with compact structure" $\mathcal{G}_{H_{X}}^{\mathrm{c}}, \mathcal{G}_{H_{Y}}^{\mathrm{c}}$ [cf. Example 2.10; [Mzk3], Appendix] associated to the geometric special fibers of the coverings corresponding to $H_{X}, H_{Y}$. Moreover, $\widehat{\alpha}_{H}$ is compatible with the natural actions of $H_{X}^{\prime} \stackrel{\text { def }}{=} \Pi_{X_{K}} /\left(H_{X} \bigcap \Delta_{X}\right), H_{Y}^{\prime} \stackrel{\text { def }}{=} \Pi_{Y_{L}} /\left(H_{Y} \bigcap \Delta_{Y}\right)$, relative to the isomorphism $\widehat{\alpha}_{H^{\prime}}: H_{X}^{\prime} \stackrel{\sim}{\rightarrow} H_{Y}^{\prime}$ induced by $\widehat{\alpha}$.

In particular, we conclude that the closed subgroups $J_{X} \subseteq H_{X} \cap \Delta_{X}^{\text {temp }} \subseteq$ $\Delta_{X}^{\text {temp }}, J_{Y} \subseteq H_{Y} \cap \Delta_{Y}^{\text {temp }} \subseteq \Delta_{Y}^{\text {temp }}$ determined by considering the pro-tempered coverings of $X_{\bar{K}}, Y_{\bar{K}}$ arising from the various tempered coverings [cf. $\S 3$ ] of $\mathcal{G}_{H_{X}}^{\mathrm{c}}, \mathcal{G}_{H_{Y}}^{\mathrm{c}}$ satisfy $\widehat{\alpha}\left(J_{X}^{\wedge}\right)=J_{Y}^{\wedge}$ [where the " $\wedge$ " denotes profinite completion, or, equivalently, closure in $\Pi_{X_{K}}, \Pi_{Y_{L}}$. Also, we note that the natural outer actions of $H_{X}^{\prime}, H_{Y}^{\prime}$ on $\pi_{1}^{\text {temp }}\left(\mathcal{G}_{H_{X}}^{\mathrm{c}}\right), \pi_{1}^{\text {temp }}\left(\mathcal{G}_{H_{Y}}^{\mathrm{c}}\right)$, respectively, determine natural isomorphisms

$$
\begin{aligned}
\Pi_{X_{K}}^{\text {temp }} / J_{X} & \stackrel{\sim}{\rightarrow} \pi_{1}^{\text {temp }}\left(\mathcal{G}_{H_{X}}^{\mathrm{c}}\right) \stackrel{\text { out }}{\rtimes} H_{X}^{\prime} \\
\Pi_{Y_{L}}^{\text {temp }} / J_{Y} & \stackrel{\sim}{\rightarrow} \pi_{1}^{\text {temp }}\left(\mathcal{G}_{H_{Y}}^{\mathrm{c}}\right) \stackrel{\text { out }}{\rtimes} H_{Y}^{\prime}
\end{aligned}
$$


which we shall use in the following discussion to identify the quotients on the left with the "outer semi-direct products" [cf. §0] on the right.

Now let us write

$$
\beta_{H}: \pi_{1}^{\text {temp }}\left(\mathcal{G}_{H_{X}}^{\mathrm{c}}\right) \stackrel{\text { out }}{\rtimes} H_{X}^{\prime} \stackrel{\sim}{\rightarrow} \pi_{1}^{\text {temp }}\left(\mathcal{G}_{H_{Y}}^{\mathrm{c}}\right) \stackrel{\text { out }}{\rtimes} H_{Y}^{\prime}
$$

for the isomorphism induced by $\widehat{\alpha}_{H}, \widehat{\alpha}_{H^{\prime}}$. The functoriality of the construction of $\widehat{\alpha}_{H}$ in [Mzk3], Lemma 2.3, implies that the profinite completion $\widehat{\beta}_{H}$ differs from the isomorphism $\Pi_{X_{K}} / J_{X}^{\wedge} \stackrel{\sim}{\rightarrow} \Pi_{Y_{L}} / J_{Y}^{\wedge}$ induced by $\widehat{\alpha}$ by composition with an inner automorphism. Also, we observe that, as one varies $H_{X}, H_{Y}$, consideration of the resulting "generalized morphisms of arithmetic graphs of anabelioids" [cf. Example 5.6, Remark 5.6.1] shows that the resulting $\beta_{H}$ 's are compatible [up to inner automorphism]. Thus, by passing to the corresponding inverse limit, we conclude that the various $\beta_{H}$ determine an isomorphism of tempered fundamental groups

$$
\beta: \Pi_{X_{K}}^{\text {temp }} \stackrel{\sim}{\rightarrow} \Pi_{Y_{L}}^{\text {temp }}
$$

whose profinite completion $\widehat{\beta}$ differs from $\widehat{\alpha}$ by an inner automorphism, as desired. That such a $\beta$ is unique, up to inner automorphism, follows from Lemma 6.1, (iii).

Remark 6.6.1. One verifies easily that the technique used in the proof of Theorem 6.6 may also be applied to give another proof of Theorem 6.5, (iii) [i.e., without resorting to the theory of $\S 3]$.

Now that we have the tempered versions - i.e., Theorems $6.4,6.5$ - of [Mzk8], Theorems 1.2, 1.3, the theory of [Mzk8], $\S 2$, concerning the category of dominant localizations $\operatorname{DLoc}_{K}\left(X_{K}\right)$ [cf. loc. cit.] generalizes in a fairly straightforward fashion to the tempered case:

First, we define the category

$$
\operatorname{DLoc}_{G_{K}}\left(\Pi_{X_{K}}^{\mathrm{temp}}\right)
$$

as follows: An object of this category is a surjection of tempered groups

$$
H \rightarrow J
$$

where $H \subseteq \Pi_{X_{K}}^{\text {temp }}$ is an open subgroup of finite index; $J$ is the quotient of $H$ by the closed normal subgroup generated by some collection of cuspidal geometric decomposition groups; and we assume that $J$ is "hyperbolic", in the sense that 
the image of $\Delta_{X}^{\text {temp }} \cap H$ in $J$ is nonabelian. Given two objects $H_{i} \rightarrow J_{i}$, where $i=1,2$, of this category, a morphism in this category is defined to be a diagram of the form

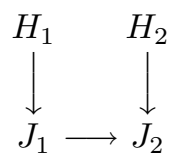

where the vertical morphisms are the given morphisms, and the horizontal morphism is an outer homomorphism of DOF-type that is compatible with the various natural [open] outer homomorphisms from the $H_{i}, J_{i}$ to $G_{K}$.

Next, let

$$
D_{x} \subseteq \Pi_{X_{K}}^{\mathrm{temp}}
$$

be a decomposition group associated to some closed point $x \in \bar{X}_{K}$.

Definition 6.7. We shall say that $x$ or $D_{x}$ is of tempered DLoc-type if $D_{x}$ admits an open subgroup that arises as the image via a morphism $Z \rightarrow X_{K}$ of $\operatorname{DLoc}_{K}\left(X_{K}\right)$ of some cuspidal decomposition group of $\Pi_{Z}^{\text {temp }}$.

Theorem 6.8 (Tempered Group-theoreticity of the Category of Dominant Localizations). Let $K, L$ be finite extensions of $\mathbb{Q}_{p} ; X_{K}$ (respectively, $\left.Y_{L}\right)$ a hyperbolic curve over $K$ (respectively, $\left.L\right)$. Then:

(i) The tempered fundamental group functor determines equivalences of categories

$$
\operatorname{DLoc}_{K}\left(X_{K}\right) \stackrel{\sim}{\rightarrow} \operatorname{DLoc}_{G_{K}}\left(\Pi_{X_{K}}^{\text {temp }}\right) ; \quad \operatorname{DLoc}_{L}\left(Y_{L}\right) \stackrel{\sim}{\rightarrow} \operatorname{DLoc}_{G_{L}}\left(\Pi_{Y_{L}}^{\text {temp }}\right)
$$

(ii) Every isomorphism of tempered groups

$$
\alpha: \Pi_{X_{K}}^{\mathrm{temp}} \stackrel{\sim}{\rightarrow} \Pi_{Y_{L}}^{\mathrm{temp}}
$$

induces an equivalence of categories

$$
\operatorname{DLoc}_{G_{K}}\left(\Pi_{X_{K}}^{\mathrm{temp}}\right) \stackrel{\sim}{\rightarrow} \operatorname{DLoc}_{G_{L}}\left(\Pi_{Y_{L}}^{\mathrm{temp}}\right)
$$

hence also [by applying the equivalences of (i)] an equivalence of categories

$$
\operatorname{DLoc}_{K}\left(X_{K}\right) \stackrel{\sim}{\rightarrow} \operatorname{DLoc}_{L}\left(Y_{L}\right)
$$

in a fashion that is functorial, up to unique isomorphisms of equivalences of categories, with respect to $\alpha$. Moreover, $\alpha$ preserves the decomposition groups of tempered DLoc-type. 
(iii) In the situation of (ii) above, suppose further that $X_{K}, Y_{L}$ are oncepunctured elliptic curves. Then $\alpha$ preserves the decomposition groups of the "torsion closed points" - i.e., the closed points that arise from torsion points of the underlying elliptic curve. Moreover, the resulting bijection between torsion closed points of $X_{K}, Y_{L}$ is compatible with the isomorphism on abelianizations of geometric fundamental groups $\Delta_{X}^{\mathrm{ab}} \stackrel{\sim}{\rightarrow} \Delta_{Y}^{\mathrm{ab}}-$ i.e., "Tate modules" - induced by $\alpha$.

(iv) In the situation of (ii) above, suppose further that $X_{K}, Y_{L}$ are isogenous [cf. $\S 0]$ to hyperbolic curves of genus zero. Then the isomorphism $\alpha$ preserves the decomposition groups of the algebraic closed points. In particular, $X_{K}$ is defined over a number field if and only if $Y_{L}$ is.

Proof. $\quad$ In light of Theorems 6.4, 6.5, the present Theorem 6.8 follows by exactly the same arguments as those applied in [Mzk8] to prove [Mzk8], Theorem 2.3; Corollaries 2.5, 2.6, 2.8.

Remark 6.8.1. Just as in the case of [Mzk8], Corollaries 2.6, 2.8, the proofs of Theorem 6.8, (iii), (iv), only require the isomorphism version of Theorem 6.4 [cf. [Mzk8], Remark 2.8.1].

Corollary 6.9 (Tempered Absoluteness of Decomposition Groups for Genus Zero). In the situation of Theorem 6.8, (iv), suppose further both $X_{K}$ and $Y_{L}$ are defined over a number field. Then the isomorphism $\alpha$ preserves the decomposition groups of all the closed points.

Proof. Corollary 6.9 follows from Theorem 6.8, (iv), by applying a similar argument to the argument used in the proof of [Mzk8], Corollary 3.2. In the present tempered case, one must therefore verify the tempered analogue of [Mzk8], Lemma 3.1. We do this as follows: First, we choose a sequence of characteristic open subgroups [cf., e.g., [André], Lemma 6.1.2, (i)]

$$
\cdots \subseteq \Delta_{X}^{\text {temp }}[j+1] \subseteq \Delta_{X}^{\text {temp }}[j] \subseteq \cdots \subseteq \Delta_{X}^{\text {temp }}
$$

[where $j$ ranges over the positive integers] of $\Delta_{X}^{\text {temp }}$ such that the $\Delta_{X}^{\text {temp }}[j]$ form a base of the topology of $\Delta_{X}^{\text {temp }}$. Next, let us observe that if $H \subseteq \Delta_{X}^{\text {temp }}$ is a characteristic open subgroup of finite index, then the open subgroup of $H$ determined by the combinatorial universal covering of the dual graph of the geometric special fiber of the covering corresponding to $H$ is still characteristic [cf. [André], Lemma 6.1.1, or, indeed, Corollary 3.11 of the present paper]. 
Thus, we may assume, without loss of generality, that [for all positive integers $j$ ] the dual graph $\mathbb{G}_{j}$ of the [geometric] special fiber of each of the tempered covering of $X_{\bar{K}}$ corresponding to $\Delta_{X}^{\text {temp }}[j]$ is a tree. In particular, given any section

$$
\sigma: G_{K} \rightarrow \Pi_{X_{K}}^{\mathrm{temp}}
$$

we obtain open subgroups

$$
\Pi_{X_{K}[j, \sigma]}^{\mathrm{temp}} \stackrel{\text { def }}{=} \operatorname{Im}(\sigma) \cdot \Delta_{X}^{\text {temp }}[j] \subseteq \Pi_{X_{K}}^{\text {temp }}
$$

[where $\operatorname{Im}(\sigma)$ denotes the image of $\sigma$ in $\Pi_{X_{K}}^{\text {temp }}$ ] corresponding to a tower of tempered coverings of $X_{K}$ :

$$
\cdots \rightarrow X_{K}[j+1, \sigma] \rightarrow X_{K}[j, \sigma] \rightarrow \cdots \rightarrow X_{K}
$$

Also, we observe that the natural action of $\Pi_{X_{K}}^{\text {temp }}$ on the tree $\mathbb{G}_{j}$ factors through $\Delta_{X[j]}^{\text {temp }}$.

Now suppose that $\operatorname{Im}(\sigma)$ is not contained in any cuspidal decomposition group of $\Pi_{X_{K}}^{\text {temp }}$. Then the following conditions on $\sigma$ are equivalent:

(i) $\sigma$ arises from a point $x \in X_{K}(K)$ [i.e., $\left." \operatorname{Im}(\sigma)=D_{x} "\right]$.

(ii) For every integer $j \geq 1, X_{K}[j, \sigma](K) \neq \emptyset$.

(iii) For every integer $j \geq 1, X_{K}[j, \sigma](K)^{\text {alg }} \neq \emptyset$ [where the superscript "alg" denotes the subset of algebraic $K$-rational points, i.e., $K$-rational points that map to algebraic points of $\left.X_{K}(K)\right]$.

(iv) For every integer $j \geq 1, \Pi_{X_{K}[j, \sigma]}^{\text {temp }}$ contains a decomposition group [i.e., relative to $\left.\Pi_{X_{K}}^{\mathrm{temp}}\right]$ of an algebraic closed point of $X_{K}$ that surjects onto $G_{K}$.

Indeed, the implications (i) $\Longrightarrow$ (ii); (iii) $\Longrightarrow$ (ii), (iv); and (iv) $\Longrightarrow$ (iii) follow formally as in the proof of [Mzk8], Lemma 3.1. Moreover, the implication (ii) $\Longrightarrow$ (iii) - i.e., "approximation via Krasner's lemma" [cf. the proof of [Mzk8], Lemma 3.1] - follows as in loc. cit., since given any point $x_{j} \in X_{K}[j, \sigma](K)$ with image $x \in X_{K}(K)$, the completion at [the $\mathcal{O}_{K}$-valued point determined by] $x_{j}$ of the normalization in $X_{K}[j, \sigma]$ of some proper model of $X_{K}$ over $\mathcal{O}_{K}$ is finite over the completion of this proper model at [the $\mathcal{O}_{K}$-valued point determined by] $x$.

Finally, we consider the implication (ii) $\Longrightarrow$ (i). In the case of loc. cit., this implication followed formally from the fact that the topological space

$$
\prod_{j \geq 1} \bar{X}_{K}[j, \sigma](K)
$$


was [in the case of loc. cit.] manifestly compact. In the present tempered case, although this compactness is not immediate, we may nevertheless conclude, at least for some cofinal set of $j$, the compactness of $\bar{X}_{K}[j, \sigma](K)$ by observing that the points of $\bar{X}_{K}[j, \sigma](K)$ always determine components [i.e., vertices or edges] of $\mathbb{G}_{j}$ that are fixed by the natural action of the image $\operatorname{Im}\left(\Pi_{X_{K}[j, \sigma]}^{\text {temp }}\right) \subseteq$ $\Pi_{X_{K}}^{\text {temp }} / \Delta_{X[j]}^{\text {temp }}$, i.e., by the natural action of $G_{K}$ on $\mathbb{G}_{j}$ via $\sigma$. On the other hand, it follows from our assumption that the $\mathbb{G}_{j}$ are trees [cf. Theorem 3.7, Theorem 5.4, and their proofs; Lemma 1.8, (ii)] that, at least for some cofinal set of $j$, this set of fixed components of $\mathbb{G}_{j}$ is finite, thus implying the desired compactness of $\bar{X}_{K}[j, \sigma](K)$.

Remark 6.9.1. $\quad$ As observed in Remark 3.7.1, the argument used in the final portion of the proof of Corollary 6.9 is reminiscent of the argument used in the "discrete real section conjecture" of [Mzk5], §3.2. This is interesting since Corollary 6.9 itself may be regarded as a weak form of the "section conjecture" for $\Pi_{X_{K}}^{\text {temp }} \rightarrow G_{K}$ [i.e., roughly speaking, the assertion that all sections of this surjection arise geometrically]. This state of affairs suggests that:

Perhaps it is more natural to consider the section conjecture for the tempered fundamental group $\Pi_{X_{K}}^{\text {temp }}$ than for its profinite completion $\Pi_{X_{K}}$.

Indeed, if one considers the section conjecture for the tempered fundamental group $\Pi_{X_{K}}^{\text {temp }}$, it seems natural to expect that the theory of arithmetically maximal compact subgroups discussed in $\S 5$ could provide useful insights that are not available in the profinite case.

Next, let

$$
D_{x} \subseteq \Pi_{X_{K}}^{\mathrm{temp}}
$$

be a decomposition group associated to some closed point $x \in \bar{X}_{K}(K)$. Then one has tempered analogues of the various notions of "absoluteness" given in [Mzk8], Definition 4.1, (iv); [Mzk8], Definition 4.8 - which we denote by means of a prefix "temp-". Observe that $D_{x}$ also forms a "profinite $D_{x} \subseteq \Pi_{X_{K}}$ " in the sense of [Mzk8]. Moreover, since finitely generated free groups are wellknown to be "good" [i.e., the natural map from the cohomology of the profinite completion of this group with coefficients in a finite [i.e., as a set] module to the cohomology of the original group in the same module is an isomorphism], it is immediate that $\Pi_{X_{K}}^{\text {temp }}$ is also good. We thus conclude [cf. Theorem 6.6] the following: 
Corollary 6.10 (Tempered Absoluteness).

(i) The point $x$ is a discretely absolute cusp (respectively, an integrally absolute cusp) if and only if it is a discretely temp-absolute cusp (respectively, an integrally temp-absolute cusp).

(ii) The hyperbolic curve $X_{K}$ is unitwise absolute if and only if it is unitwise temp-absolute.

Corollary 6.11 (Unitwise and Integral Temp-absoluteness for Genus Zero). Let $X_{K}$ be a hyperbolic curve over $K$, with stable reduction over $\mathcal{O}_{K}$, which is isogenous to a hyperbolic curve of genus zero. Then $X_{K}$ is unitwise temp-absolute, and every cusp of $X_{K}$ is integrally tempabsolute.

Proof. Indeed, this follows by formally "substituting" the equivalences of Corollary 6.10, (i), (ii), into [Mzk8], Corollary 4.11.

Finally, we also observe that it is immediate that the tempered analogue of [Mzk8], Theorem 4.3, holds:

Theorem 6.12 (Rigidity of Cuspidal Geometric Decomposition Groups). In the notation of Theorem 6.8, (ii), suppose that $\alpha$ induces isomorphisms

$$
I_{x} \stackrel{\sim}{\rightarrow} I_{y} ; \quad \boldsymbol{\mu}_{\widehat{\mathbb{Z}}}(\bar{K}) \stackrel{\sim}{\rightarrow} \boldsymbol{\mu}_{\widehat{\mathbb{Z}}}(\bar{L})
$$

where $x \in \bar{X}_{K}(K)$ (respectively, $y \in \bar{Y}_{L}(L)$ ) is a cusp; $\boldsymbol{\mu}_{\widehat{\mathbb{Z}}}(-)$ is as in [Mzk8], Theorem 4.3. Then these isomorphisms are compatible with the natural isomorphisms $\boldsymbol{\mu}_{\widehat{\mathbb{Z}}}(\bar{K}) \stackrel{\sim}{\rightarrow} I_{x} ; \boldsymbol{\mu}_{\widehat{\mathbb{Z}}}(\bar{L}) \stackrel{\sim}{\rightarrow} I_{y}$.

Remark 6.12.1. Note that unlike Corollaries 6.10, 6.11; Theorem 6.12, results such as Theorem 6.8, (iii), (iv); Corollary 6.9 do not follow formally from their profinite analogues, since, in the latter case, it is by no means clear that any $\Pi_{X_{K}}$-conjugate of the decomposition group of a closed point that happens to be contained in $\Pi_{X_{K}}^{\text {temp }} \subseteq \Pi_{X_{K}}$ is necessarily a $\Pi_{X_{K}}^{\text {temp }}$-conjugate of the decomposition group of a closed point.

\section{Appendix : Quasi-temperoids}

In this Appendix, we discuss a certain minor generalization of the notion of a temperoid introduced in $\S 3$. 
Let $\mathcal{T}$ be a temperoid. If $A$ is an object of $\mathcal{T}$, write $\pi_{0}(A)$ for the set of connected components of $A$ and

$$
\mathcal{T}[A] \subseteq \mathcal{T}
$$

for the full subcategory determined by the objects of $\mathcal{T}$ that admit a morphism to $A[\mathrm{cf} \S 0]$. This Appendix is devoted to the resolution [cf. Theorem A.4 below] of the following question:

Given the abstract category $\mathcal{T}[A]$, is it possible to reconstruct the category $\mathcal{T}$ category-theoretically from $\mathcal{T}[A]$ ?

This sort of issue occurs naturally, for instance, in the study of cores of anabelioids, and, indeed, is addressed implicitly in [Mzk4], Proposition 2.1.1, (iii). Since, however, this sort of technical issue is a bit more technically complicated in the case of temperoids than in the case of anabelioids, and, moreover, even in the case of anabelioids, this sort of issue is not addressed explicitly or in detail in [Mzk4], it seemed appropriate to the author to give the details in the present Appendix of how this sort of issue may be resolved.

Definition A.1. (i) Any category equivalent to a category of the form

$$
\mathcal{T}[A]
$$

— where $A$ is a connected object, and $\mathcal{T}$ is a connected temperoid — will be referred to as a connected quasi-temperoid.

(ii) A category equivalent to a product [in the sense of a product of categories] of a countable [hence possibly empty!] collection of connected quasitemperoids will be referred to as a quasi-temperoid. An object $A$ of a quasitemperoid $\mathcal{Q}$ will be called nondegenerate if, for every connected object $B$ of $\mathcal{Q}$, there exist arrows $C \rightarrow B, C \rightarrow A$, for some connected object $C$ of $\mathcal{Q}$.

(iii) Let $\mathcal{Q}_{1}, \mathcal{Q}_{2}$ be quasi-temperoids. Then a quasi-morphism $\phi: \mathcal{Q}_{1} \rightarrow \mathcal{Q}_{2}$ is defined to be a functor $\phi^{*}: \mathcal{Q}_{2} \rightarrow \mathcal{Q}_{1}$ that preserves finite limits and countable colimits. A quasi-morphism $\phi$ will be called rigid (respectively, a morphism) if the functor $\phi^{*}$ is rigid [cf. $\S 0$ ] (respectively, preserves nondegenerate objects).

Remark A.1.1. One verifies immediately that a quasi-temperoid is an almost totally epimorphic category of countably connected type [cf. §0]. 
Remark A.1.2. Unlike the situation with temperoids, a quasi-temperoid does not, in general, admit a terminal object. Thus, it is not in general the case that a quasi-morphism of quasi-temperoids is necessarily a morphism. Indeed, if $\mathcal{Q}$ is a connected quasi-temperoid that does not admit a terminal object, then one verifies immediately that the functor $\mathcal{Q} \rightarrow \mathcal{Q}$ that maps all objects of $\mathcal{Q}$ to some empty object of $\mathcal{Q}$ preserves finite limits and countable colimits, but fails to preserve nondegenerate objects.

Proposition A.2 (Connected Components of Quasi-temperoids). Let $E, E^{\prime}$ be countable sets; for each $e \in E$ (respectively, $\left.e^{\prime} \in E^{\prime}\right)$, let $\mathcal{Q}_{e}$ (respectively, $\left.\mathcal{Q}_{e^{\prime}}^{\prime}\right)$ be a connected quasi-temperoid; set:

$$
\mathcal{Q} \stackrel{\text { def }}{=} \prod_{e \in E} \mathcal{Q}_{e} ; \quad \mathcal{Q}^{\prime} \stackrel{\text { def }}{=} \prod_{e^{\prime} \in E^{\prime}} \mathcal{Q}_{e^{\prime}}^{\prime}
$$

Also, let $\phi: \mathcal{Q} \rightarrow \mathcal{Q}^{\prime}$ be a quasi-morphism of quasi-temperoids. Then:

(i) For each $e \in E$, the natural projection functor

$$
\pi_{e}^{*}: \mathcal{Q} \rightarrow \mathcal{Q}_{e}
$$

determines a morphism of quasi-temperoids $\mathcal{Q}_{e} \rightarrow \mathcal{Q}$.

(ii) For each $e \in E$, write

$$
\iota_{e}: \mathcal{Q}_{e} \rightarrow \mathcal{Q}
$$

for the natural inclusion functor [i.e., the functor whose composite with $\pi_{f}^{*}$, where $f \in E$, maps all objects of $\mathcal{Q}_{e}$ to empty [i.e., initial] objects of $\mathcal{Q}_{f}$ if $f \neq e$, and is the identity if $f=e]$. If $\epsilon$ is a connected component of the full subcategory of connected objects $\mathcal{Q}^{0} \subseteq \mathcal{Q}[c f$. $\S 0]$, then write

$$
\mathcal{Q}_{\epsilon} \subseteq \mathcal{Q}
$$

for the full subcategory determined by the objects $A$ of $\mathcal{Q}$ such that all of the connected components of $A$ belong to $\epsilon$. Then the essential image of $\iota_{e}$ is equal to $\mathcal{Q}_{\epsilon}$ for a unique $\epsilon$, and, moreover, the resulting correspondence

$$
e \mapsto \epsilon
$$

determines a bijection between $E$ and the set of connected components of $\mathcal{Q}^{0}$. 
(iii) The nondegenerate objects of $\mathcal{Q}$ are precisely the objects each of whose component objects $\in \mathrm{Ob}\left(\mathcal{Q}_{e}\right)$ is nonempty.

(iv) If $\phi$ is a morphism of quasi-temperoids, and both $E$ and $E^{\prime}$ are of cardinality one, then the functor $\phi^{*}$ is faithful.

(v) If $\phi$ is a morphism of quasi-temperoids, then $\phi$ induces a map $\psi: E \rightarrow E^{\prime}$, and, for each $e \in E$, a morphism of quasi-temperoids $\phi_{e}: \mathcal{Q}_{e} \rightarrow$ $\mathcal{Q}_{\psi(e)}^{\prime}$ such that $\phi$ coincides with the morphism of quasi-temperoids formed by "taking the product" [in the evident sense] of the $\phi_{e}$.

Proof. Assertion (i) (respectively, (iii)) follows immediately from the definitions (respectively, and assertion (ii)). To prove assertion (ii), we argue as follows: By unraveling the definitions, one verifies immediately that every connected object of $\mathcal{Q}$ lies in the essential image of a unique $\iota_{e}$, and that two connected objects of $\mathcal{Q}$ lie in the essential image of the same $\iota_{e}$ if and only if they belong to the same connected component of $\mathcal{Q}^{0}$. Now assertion (ii) follows formally from these observations.

Next, we verify assertion (iv). First, in light of Remark A.1.1, it suffices to check faithfulness on arrows $A \rightarrow B$ between connected objects $A, B$ of $\mathcal{Q}^{\prime}$. But since $A, B$ are connected, it follows immediately that there exists a connected object $C$ of $\mathcal{Q}^{\prime}$ such that the products $A \times C, B \times C$ split as coproducts of copies of $C$. Since $A, B, C$ are connected [hence nonempty], they are nondegenerate [cf. assertion (iii)]. Moreover, again by Remark A.1.1, arrows $A \rightarrow B$ are represented faithfully by the maps $\pi_{0}(A \times C) \rightarrow \pi_{0}(B \times C)$ they induce. But, since $\phi^{*}(A), \phi^{*}(B), \phi^{*}(C)$ are nondegenerate, hence, in particular, nonempty, this implies that $\phi^{*}$ itself is faithful, as desired.

Finally, we verify assertion (v). Let us consider the various composite functors

$$
\kappa\left[e^{\prime}, e\right] \stackrel{\text { def }}{=} \pi_{e}^{*} \circ \phi^{*} \circ \iota_{e^{\prime}}: \mathcal{Q}_{e^{\prime}}^{\prime} \rightarrow \mathcal{Q}_{e}
$$

where $e \in E, e^{\prime} \in E^{\prime}$. Now observe that, if we fix $e$ and allow $e^{\prime}$ to vary, then since the product of objects belonging to distinct $\mathcal{Q}_{\epsilon^{\prime}}^{\prime}$ 's will always be an empty object of $\mathcal{Q}^{\prime}$, it follows from the fact that $\pi_{e}, \phi$ are quasi-morphisms of quasi-temperoids [together with the easily verified observation that any product of nonempty objects of a connected quasi-temperoid will always be nonempty] that there is at most one $e^{\prime} \in E^{\prime}$ such that the essential image of $\kappa\left[e^{\prime}, e\right]$ contains nonempty objects. On the other hand, since $\pi_{e}^{*} \circ \phi^{*}$ preserves nondegenerate objects, it follows [cf. assertion (iii)] that there exists at least one $e^{\prime} \in E^{\prime}$ such that the essential image of $\kappa\left[e^{\prime}, e\right]$ contains nonempty objects. Thus, in summary, 
for each $e \in E$, there exists a unique $e^{\prime} \in E^{\prime}$ such that the essential image of $\kappa\left[e^{\prime}, e\right]$ contains nonempty objects; set $\psi(e) \stackrel{\text { def }}{=} e^{\prime}$. Then it follows immediately again from the fact that the functor $\pi_{e}^{*} \circ \phi^{*}$ preserves nondegenerate objects that the functor $\kappa[\psi(e), e]$ preserves nondegenerate objects, hence determines a morphism of quasi-temperoids $\mathcal{Q}_{e} \rightarrow \mathcal{Q}_{\psi(e)}^{\prime}$. Moreover, unraveling the definitions, we see that the remainder of assertion (v) follows formally from what we have done thus far.

Remark A.2.1. Thus, Proposition A.2 serves, in effect, to reduce the theory of arbitrary quasi-temperoids to the theory of connected quasi-temperoids.

Definition A.3. Let $\mathcal{Q}$ be a connected quasi-temperoid. Then:

(i) Any pair $\left(A, \Gamma_{A}\right)$, where $A$ is an object of $\mathcal{Q}$, and $\Gamma_{A} \subseteq \operatorname{Aut}_{\mathcal{Q}}(A)$ is a subgroup, will be referred to as a $Q D$ - [or quotient data] pair [of $\mathcal{Q}$ ]. If $\Gamma_{A}$ acts transitively on $\pi_{0}(A)$, then we shall say that this QD-pair is weakly connected; if $A$ is connected, then we shall say that this QD-pair is strongly connected. [Thus, every strongly connected QD-pair is weakly connected.]

(ii) A morphism of $Q D$-pairs of $\mathcal{Q}$

$$
\left(A, \Gamma_{A}\right) \rightarrow\left(B, \Gamma_{B}\right)
$$

is defined to be a morphism $\phi: A \rightarrow B$ such that, for every $\gamma_{A} \in \Gamma_{A}$, there exists a $\gamma_{B} \in \Gamma_{B}$ such that $\gamma_{B} \circ \phi=\phi \circ \gamma_{A}$. If, moreover, $\phi$ induces a surjection $\pi_{0}(A) \rightarrow \pi_{0}(B)$, then we shall say that this morphism is 0-proper. Thus, in the 0-proper case, it follows from the fact that the category $\mathcal{Q}$ is almost totally epimorphic that $\gamma_{B}$ is unique, hence that the correspondence $\gamma_{A} \mapsto \gamma_{B}$ determines an associated group homomorphism $\Gamma_{A} \rightarrow \Gamma_{B}$.

(iii) Let $\left(A, \Gamma_{A}\right)$ be a QD-pair. Then we shall say that an arrow $\phi: A \rightarrow B$ of $\mathcal{Q}$ forms a quotient of this $Q D$-pair - and write $B \cong A / \Gamma_{A}$ - if the following two properties are satisfied: (a) $\phi \circ \gamma_{A}=\phi, \forall \gamma_{A} \in \Gamma_{A}$; (b) for every arrow $\psi_{A}: A \rightarrow C$ satisfying $\psi_{A} \circ \gamma_{A}=\psi_{A}, \forall \gamma_{A} \in \Gamma_{A}$, there exists a unique arrow $\psi_{B}: B \rightarrow C$ such that $\psi_{B} \circ \phi=\psi_{A}$. [Thus, one verifies immediately that the quotient of a QD-pair is unique, up to unique isomorphism, if it exists, and that the quotient of a QD-pair is connected if and only if the QD-pair is weakly connected.]

(iv) A 0 -proper morphism of QD-pairs of $\mathcal{Q}$

$$
\left(A, \Gamma_{A}\right) \rightarrow\left(B, \Gamma_{B}\right)
$$


will be called 1-proper if the associated homomorphism $\Gamma_{A} \rightarrow \Gamma_{B}$ is surjective, and, moreover, the arrow $A \rightarrow B$ forms a quotient of the QD-pair $\left(A, \operatorname{Ker}\left(\Gamma_{A} \rightarrow\right.\right.$ $\left.\Gamma_{B}\right)$ ). [Thus, under the 1 -properness assumption, one verifies immediately that if $B \rightarrow C$ forms a quotient of $\left(B, \Gamma_{B}\right)$, then the composite arrow $A \rightarrow B \rightarrow C$ forms a quotient of $\left(A, \Gamma_{A}\right)$.]

Remark A.3.1. One verifies immediately that the following conditions on a morphism of QD-pairs $\left(A, \Gamma_{A}\right) \rightarrow\left(B, \Gamma_{B}\right)$ are equivalent:

(a) $\left(A, \Gamma_{A}\right) \rightarrow\left(B, \Gamma_{B}\right)$ is 0-proper.

(b) $A \rightarrow B$ is an epimorphism in $\mathcal{Q}$ [cf. Remark A.1.1].

(c) $B$ is the colimit in $\mathcal{Q}$ of the diagram formed by the two projections $A \times_{B}$ $A \rightarrow A$.

Moreover, the notion of a "quotient" given in Definition A.3, (iii), may also be stated in terms of colimits. In particular, the condition that $\left(A, \Gamma_{A}\right) \rightarrow\left(B, \Gamma_{B}\right)$ be 1-proper may be stated entirely in terms of colimits.

Theorem A.4 (Connected Quasi-temperoids). For $i=1,2$, let $\mathcal{T}_{i}$ be a connected temperoid; let $A_{i}$ be a connected object of $\mathcal{T}_{i}$; write

$$
\lambda_{i}: \mathcal{Q}_{i} \stackrel{\text { def }}{=} \mathcal{T}_{i}\left[A_{i}\right] \rightarrow \mathcal{T}_{i}
$$

for the natural functor. Then any morphism of quasi-temperoids $\phi: \mathcal{Q}_{1} \rightarrow$ $\mathcal{Q}_{2}$ fits into a 1-commutative diagram

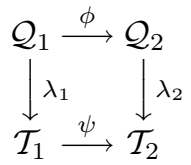

- where the morphism of [quasi-] temperoids $\psi: \mathcal{T}_{1} \rightarrow \mathcal{T}_{2}$ that makes this diagram 1-commute is unique, up to unique isomorphism.

Proof. Write

$$
\mathcal{D}_{i}
$$

for the category whose objects are QD-pairs of $\mathcal{Q}_{i}$ and whose morphisms are morphisms of QD-pairs. Note that it follows immediately from the definitions that if $\left(B, \Gamma_{B}\right)$ is a QD-pair of $\mathcal{Q}_{i}$, then the QD-pair $\lambda_{i}\left(B, \Gamma_{B}\right)$ of $\mathcal{T}_{i}$ admits a quotient in $\mathcal{T}_{i}$. Thus, we obtain a natural functor

$$
q_{i}: \mathcal{D}_{i} \rightarrow \mathcal{T}_{i}
$$


given by the assignment $\left(B, \Gamma_{B}\right) \mapsto B / \Gamma_{B}$. Note that this functor maps 1 proper morphisms of $\mathcal{D}_{i}$ to isomorphisms of $\mathcal{T}_{i}$. Moreover, one verifies immediately that this functor $q_{i}$ is essentially surjective; that $q_{i}\left(\left(B, \Gamma_{B}\right)\right)$ is connected if and only if $\left(B, \Gamma_{B}\right)$ is weakly connected; and that every connected object of $\mathcal{T}_{i}$ is isomorphic to the image via $q_{i}$ of a strongly connected QD-pair.

Thus, to reconstruct $\mathcal{T}_{i}$ from $\mathcal{D}_{i}$, it suffices to reconstruct the morphisms

$$
B / \Gamma_{B} \rightarrow C / \Gamma_{C}
$$

in $\mathcal{T}_{i}$ between the images via $q_{i}$ of two objects $\left(B, \Gamma_{B}\right),\left(C, \Gamma_{C}\right)$ of $\mathcal{D}_{i}$. To this end, we define

$$
\underline{\operatorname{Hom}}\left(\left(B, \Gamma_{B}\right),\left(C, \Gamma_{C}\right)\right) \stackrel{\text { def }}{=} \operatorname{Hom}_{\mathcal{D}_{i}}\left(\left(B, \Gamma_{B}\right),\left(C, \Gamma_{C}\right)\right) / \Gamma_{C}
$$

[i.e., where $\Gamma_{C}$ acts by composition from the right], so that the functor $q_{i}$ induces a natural map:

$$
\underline{\operatorname{Hom}}\left(\left(B, \Gamma_{B}\right),\left(C, \Gamma_{C}\right)\right) \rightarrow \operatorname{Hom}_{\mathcal{T}_{i}}\left(B / \Gamma_{B}, C / \Gamma_{C}\right)
$$

Since $\mathcal{T}_{i}$ is an almost totally epimorphic category countably connected type, it suffices to describe the morphisms between connected objects of $\mathcal{T}_{i}$.

Next, suppose that $\left(B, \Gamma_{B}\right),\left(C, \Gamma_{C}\right)$ are strongly connected $Q D$-pairs. Then observe that the functor $q_{i}$ induces an injection:

$$
\underline{\operatorname{Hom}}\left(\left(B, \Gamma_{B}\right),\left(C, \Gamma_{C}\right)\right) \hookrightarrow \operatorname{Hom}_{\mathcal{T}_{i}}\left(B / \Gamma_{B}, C / \Gamma_{C}\right)
$$

Indeed, this follows immediately by considering the natural splitting [in $\mathcal{Q}_{i}$ or $\mathcal{T}_{i}$ ] of $C \times{ }_{C / \Gamma_{C}} C$ into a coproduct of copies of $C$ indexed by $\Gamma_{C}$ [together with the fact that $\left(B, \Gamma_{B}\right),\left(C, \Gamma_{C}\right)$ are strongly connected $Q D$-pairs]. Moreover, one verifies immediately that every element of $\operatorname{Hom}_{\mathcal{T}_{i}}\left(B / \Gamma_{B}, C / \Gamma_{C}\right)$ arises from some morphism of strongly connected QD-pairs

$$
\left(B^{\prime}, \Gamma_{B^{\prime}}\right) \rightarrow\left(C, \Gamma_{C}\right)
$$

where $\left(B^{\prime}, \Gamma_{B^{\prime}}\right) \rightarrow\left(B, \Gamma_{B}\right)$ is a 1-proper morphism of strongly connected QDpairs [so we obtain a morphism $B / \Gamma_{B} \rightarrow C / \Gamma_{C}$ by composing the induced morphism $B^{\prime} / \Gamma_{B^{\prime}} \rightarrow C / \Gamma_{C}$ with the inverse of the induced isomorphism $\left.B^{\prime} / \Gamma_{B^{\prime}} \stackrel{\sim}{\rightarrow} B / \Gamma_{B}\right]$. Now one verifies easily that any two 1-proper morphisms of strongly connected QD-pairs $\left(B^{\prime}, \Gamma_{B^{\prime}}\right) \rightarrow\left(B, \Gamma_{B}\right),\left(B^{\prime \prime}, \Gamma_{B^{\prime \prime}}\right) \rightarrow\left(B, \Gamma_{B}\right)$ fit into a commutative diagram of 1-proper morphisms of strongly connected QD-pairs of $\mathcal{D}_{i}$ :

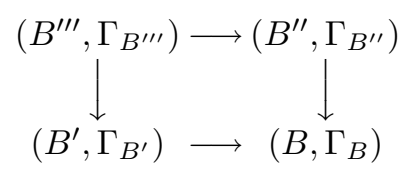


Thus, we conclude that $\operatorname{Hom}_{\mathcal{T}_{i}}\left(B / \Gamma_{B}, C / \Gamma_{C}\right)$ may be reconstructed as the following filtered inductive limit:

$$
\underline{\underline{\operatorname{Hom}}}\left(\left(B, \Gamma_{B}\right),\left(C, \Gamma_{C}\right)\right) \stackrel{\text { def }}{=} \underline{\lim } \underline{\operatorname{Hom}}\left(\left(B^{\prime}, \Gamma_{B^{\prime}}\right),\left(C, \Gamma_{C}\right)\right)
$$

[i.e., over 1-proper morphisms of strongly connected QD-pairs $\left(B^{\prime}, \Gamma_{B^{\prime}}\right) \rightarrow$ $\left(B, \Gamma_{B}\right)$ and transition morphisms $\left(B^{\prime \prime}, \Gamma_{B^{\prime \prime}}\right) \rightarrow\left(B^{\prime}, \Gamma_{B^{\prime}}\right)$ over $\left.\left(B, \Gamma_{B}\right)\right]$. Moreover, one verifies immediately that this reconstruction is compatible with composition of arrows [i.e., composition of arrows in $\mathcal{D}_{i}$ induces composition of "Hom-arrows"].

Next, suppose that $\left(B, \Gamma_{B}\right),\left(C, \Gamma_{C}\right)$ are weakly connected $Q D$-pairs. Then observe that each connected component $B^{\prime}$ of $B$ determines a strongly connected QD-pair $\left(B^{\prime}, \Gamma_{B^{\prime}}\right)$ [i.e., where we take $\Gamma_{B^{\prime}} \subseteq \Gamma_{B}$ to be the subgroup of automorphisms that fix the element $\left[B^{\prime}\right] \in \pi_{0}(B)$ determined by $\left.B^{\prime}\right]$ such that $q_{i}\left(\left(B^{\prime}, \Gamma_{B^{\prime}}\right)\right) \cong q_{i}\left(\left(B, \Gamma_{B}\right)\right)$; a similar statement holds for $\left(C, \Gamma_{C}\right)$. Moreover, if $B^{\prime}, B^{\prime \prime}$ (respectively, $C^{\prime}, C^{\prime \prime}$ ) are connected components of $B$ (respectively, $C$ ), then one verifies immediately that any choice of elements $\gamma_{B} \in \Gamma_{B}, \gamma_{C} \in \Gamma_{C}$ such that $\gamma_{B}\left(B^{\prime}\right)=B^{\prime \prime}, \gamma_{C}\left(C^{\prime}\right)=C^{\prime \prime}$ determines a bijection

$$
\underline{\underline{\operatorname{Hom}}}\left(\left(B^{\prime}, \Gamma_{B^{\prime}}\right),\left(C^{\prime}, \Gamma_{C^{\prime}}\right)\right) \stackrel{\sim}{\rightarrow} \underline{\underline{\operatorname{Hom}}}\left(\left(B^{\prime \prime}, \Gamma_{B^{\prime \prime}}\right),\left(C^{\prime \prime}, \Gamma_{C^{\prime \prime}}\right)\right)
$$

which is, in fact, independent of the choice of $\gamma_{B}, \gamma_{C}$. Thus, if we define

$$
\underline{\underline{\operatorname{Hom}}}\left(\left(B, \Gamma_{B}\right),\left(C, \Gamma_{C}\right)\right) \subseteq \prod_{B^{\prime}, C^{\prime}} \underline{\underline{\operatorname{Hom}}}\left(\left(B^{\prime}, \Gamma_{B^{\prime}}\right),\left(C^{\prime}, \Gamma_{C^{\prime}}\right)\right)
$$

[where the product ranges over all choices of connected components $B^{\prime}, C^{\prime}$ of $B$, $C$, respectively] to be the subset of collections of elements that correspond via these bijections, then the natural projections of this direct product determine bijections as follows:

$$
\begin{aligned}
\underline{\underline{\operatorname{Hom}}}\left(\left(B, \Gamma_{B}\right),\left(C, \Gamma_{C}\right)\right) & \stackrel{\sim}{\rightarrow} \underline{\underline{\operatorname{Hom}}}\left(\left(B^{\prime}, \Gamma_{B^{\prime}}\right),\left(C^{\prime}, \Gamma_{C^{\prime}}\right)\right) \\
& \stackrel{\sim}{\rightarrow} \operatorname{Hom}_{\mathcal{T}_{i}}\left(q_{i}\left(\left(B^{\prime}, \Gamma_{B^{\prime}}\right)\right), q_{i}\left(\left(C^{\prime}, \Gamma_{C^{\prime}}\right)\right)\right) \\
& \stackrel{\sim}{\rightarrow} \operatorname{Hom}_{\mathcal{T}_{i}}\left(q_{i}\left(\left(B, \Gamma_{B}\right)\right), q_{i}\left(\left(C, \Gamma_{C}\right)\right)\right)
\end{aligned}
$$

Moreover, it follows immediately from the definitions that we have a natural map:

$$
\underline{\operatorname{Hom}}\left(\left(B, \Gamma_{B}\right),\left(C, \Gamma_{C}\right)\right) \rightarrow \underline{\underline{\operatorname{Hom}}}\left(\left(B, \Gamma_{B}\right),\left(C, \Gamma_{C}\right)\right)
$$

Finally, as observed above, since $\mathcal{T}_{i}$ is an almost totally epimorphic category countably connected type, the definition of "Hom", as well as the resulting bijection of "Hom" with "Hom $\mathcal{T}_{i}$ " and the natural map from "ㅂom" to "Hom" 
extend immediately to pairs of objects of $\mathcal{D}_{i}$ that are not necessarily weakly connected.

Thus, in summary, if we write

$$
\mathcal{P}_{i}
$$

for the category whose objects are the objects of $\mathcal{D}_{i}$ and whose morphisms are given by the "Hom's", then we obtain natural functors

$$
\mathcal{Q}_{i} \rightarrow \mathcal{D}_{i} \rightarrow \mathcal{P}_{i} \stackrel{\sim}{\rightarrow} \mathcal{T}_{i}
$$

- i.e., the first functor maps an object $B$ of $\mathcal{Q}_{i}$ to the QD-pair $(B,\{1\})$; the second functor arises from the construction of $\mathcal{P}_{i}$; the third functor is the equivalence induced by the natural functor $\mathcal{D}_{i} \rightarrow \mathcal{T}_{i}$ considered above.

Now observe that the functor $\phi^{*}: \mathcal{Q}_{2} \rightarrow \mathcal{Q}_{1}$ induces a 1-commutative diagram

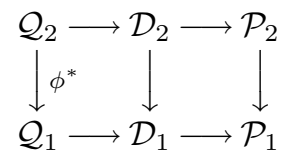

Indeed, the construction of the second vertical arrow is immediate from the definitions. The construction of the third vertical arrow follows by observing that since $\phi^{*}$ preserves countable colimits, it follows that the functor $\mathcal{D}_{2} \rightarrow \mathcal{D}_{1}$ preserves 0 - and 1-proper morphisms [cf. Remark A.3.1]. Thus, by combining this diagram with the equivalences $\mathcal{P}_{i} \stackrel{\sim}{\rightarrow} \mathcal{T}_{i}$, we obtain a diagram as in the statement of Theorem A.4.

The fact that the resulting functor $\psi^{*}: \mathcal{T}_{2} \rightarrow \mathcal{T}_{1}$ preserves countable colimits (respectively, fibered products) follows by a routine argument from the fact that $\phi^{*}$ preserves countable colimits (respectively, countable colimits and finite limits). Thus, to show that $\psi$ is a morphism of temperoids [i.e., that $\psi^{*}$ preserves finite limits], it suffices to show that $\psi^{*}$ preserves terminal objects. Now let $B$ be a connected object of $\mathcal{Q}_{2}$ such that $B \times B$ splits as a coproduct of copies of $B$ [i.e., in other words, $B$ is a connected Galois object of $\mathcal{T}_{2}$ that admits a morphism in $\mathcal{T}_{2}$ to $\left.A_{2}\right]$. Note that such a $B$ always exists. Then if we let $\operatorname{Aut}(B)$ act on, say, the second factor of $B \times B$, then the resulting QD-pair $(B \times B, \operatorname{Aut}(B))$ maps via $\phi^{*}$ to a $\mathrm{QD}$-pair $\left(\phi^{*}(B) \times \phi^{*}(B), \phi^{*}(\operatorname{Aut}(B))\right)$ of $\mathcal{Q}_{1}$. Moreover, since the first projection $B \times B \rightarrow B$ forms a quotient of the former QD-pair, it follows that the first projection $\phi^{*}(B) \times \phi^{*}(B) \rightarrow \phi^{*}(B)$ forms a quotient of the latter QD-pair. It thus follows immediately that $\phi^{*}(\operatorname{Aut}(B))$ acts transitively on $\pi_{0}\left(\phi^{*}(B)\right)$. Next, observe that $B \times B$ is a coproduct of copies of $B$. Thus, we conclude that $\phi^{*}(B) \times \phi^{*}(B)$ is a coproduct of copies of $\phi^{*}(B)$. 
But this implies that the connected components of $\phi^{*}(B)$, all of which are isomorphic to one another [by the transitivity observed above], form Galois objects of $\mathcal{T}_{1}$. Thus, the preceding observation concerning the quotient of the QD-pair in $\mathcal{Q}_{1}$ implies that the stabilizer in $\phi^{*}(\operatorname{Aut}(B))$ of any connected component $\phi^{*}(B)$ is necessarily equal to the entire automorphism group of this connected component. Moreover, since $\phi^{*}$ preserves nondegenerate objects, it follows that $\phi^{*}(B)$ is nonempty [i.e., $\phi^{*}(B)$ has at least one connected component]. Thus, in summary, $\phi^{*}$ maps the QD-pair $(B, \operatorname{Aut}(B))$ [any quotient of which forms a terminal object in $\left.\mathcal{T}_{2}\right]$ to a QD-pair $\left(\phi^{*}(B), \phi^{*}(\operatorname{Aut}(B))\right)$ any quotient of which forms a terminal object in $\mathcal{T}_{1}$. That is to say, we have shown that $\psi^{*}$ preserves terminal objects, as desired.

Finally, the asserted uniqueness of $\psi$ [up to unique isomorphism] follows immediately from the fact that arbitrary objects of $\mathcal{T}_{i}$ may be obtained as quotients of QD-pairs of $\mathcal{Q}_{i}$.

Remark A.4.1. Thus, Theorem A.4 serves, in effect, to reduce the theory of arbitrary connected quasi-temperoids to the theory of connected temperoids.

Remark A.4.2. One verifies immediately that, by replacing the term "temperoid" by the term "anabelioid" [and the terms "countable/countably" by the terms "finite/finitely"], one obtains an entirely analogous [but, in fact, slightly easier] theory of "quasi-anabelioids" to the theory of quasi-temperoids developed above. We leave the routine details to the reader.

\section{Acknowledgements}

I would like to thank Akio Tamagawa and Makoto Matsumoto for many helpful comments concerning the material presented in this paper. In particular, I am indebted to A. Tamagawa for informing me of Corollary 1.6 and the reference quoted in Remark 1.7.1, and to M. Matsumoto for informing me of his proof of Theorem 1.2.

\section{Index}

0-commutative, $§ 0$, Categories

0 -proper, A.3, ii

1-commutative, $\S 0$, Categories

1-proper, A.3, iv 
abstract equivalence, $\S 0$, Categories

abut, $\S 1$

action, 5.1, i

almost totally epimorphic, $\S 0$, Categories

aloof (totally), 2.4, iv

anabelioids, before 2.1

approximator, 2.3 , ii

arithmetic action, 5.1, ii

arithmetically ample, 5.3, i

arithmetically estranged (totally), 5.3, ii

arithmetically maximal, 5.3 , i

arithmetic component, 5.1 , ii

arithmetic semi-graph of anabelioids

(connected, connected component of, geometric component of), 5.1, ii, iii

BC (base of constants), 5.1, iv

bounded order, 2.3 , i

branch, $\S 1$

category of dominant localizations, after 6.6.1

category of localizations, after 4.1.2

category of quasi-connected objects, $\S 0$, Categories

centralizer, $§ 0$, Topological Groups

closed edge, $\S 1$

closed object, after 4.1.2

coherent, 2.3, iii

coincidence map, $\S 1$

combinatorial universal covering, after 3.5.2

commensurably terminal, $\S 0$, Topological Groups

commensurator, §0, Topological Groups

compactification (of a curve), §0, Curves

compactification of a semi-graph, $\S 1$

component of a semi-graph, $\S 1$

connected (component of a) category, §0, Categories

connected semi-graph of anabelioids, after 2.1

constituent anabelioids, 2.1

continuous action, 5.1 , i

countable semi-graph, $\S 1$ 
countable semi-graph of anabelioids, after 3.4.2

countably connected type, $\S 0$, Categories

covering of a semi-graph of anabelioids, 3.5, i

coverticial edges, $\S 1$

cuspidal (geometric) decomposition group, after 6.4

DFG-type, 6.2, i, iii

divisor at infinity, $\S 0$, Curves

divisor of cusps, $\S 0$, Curves

divisor of marked points, $\S 0$, Curves

DOF-type, 6.2, ii, iii

edge, $\S 1$

edge-like subgroup, 3.7, iii

edge-like subgroup (arithmetic case), 5.3, iii

edge-wise length, 4.2, i

elevated (totally), 2.4, i

embedding (of semi-graphs), $\S 1$

embedding (of semi-graphs of anabelioids), 4.1, ii

estranged (totally), 2.4, iv

étale morphism of temperoids, 3.4 , i

excision (of semi-graphs), $\S 1$

excision (of semi-graphs of anabelioids), 4.1, ii

family of (hyperbolic) curves, $\S 0$, Curves

finite étale covering of semi-graph of anabelioids, 2.2, i

finitely connected type, $\S 0$, Categories

finite open object, after 4.1 .2

finite semi-graph, $\S 1$

Galois covering of a semi-graph of anabelioids, 3.5, iii

Galois object (of a temperoid), 3.1, iv

$\mathbb{G}$-closed, 4.1.1

generalized morphism of semi-graphs of anabelioids, 2.11

geometric component, 5.1, ii

$(\mathcal{G}, \Gamma)$-structure, 4.1 , iii

$\mathbb{G}$-open, 4.1.1

graph, $\S 1$

graph-covering (finite, Galois), $§ 1$ 
graph-localization morphism (strict), 4.2, ii

immersion (of semi-graphs), $\S 1$

immersion (of semi-graphs of anabelioids), 4.1, ii

immobile, §0, Categories

indissectible , §0, Categories

infinite open object, after 4.1.2

injective type, 2.1

inner action, 5.1, i

iso-excisive, 4.1, iii, iv

iso-immersive, 4.1 , iii, iv

isolated edge, $\S 1$

isomorphism between functors, $§ 0$, Categories

joint, $\S 1$

link, 4.2, i

local $(\mathcal{G}, \Gamma)$-structure, 4.1 , iv

locally finite étale morphism of semi-graphs of anabelioids, 2.2, ii

locally finite semi-graph, $\S 1$

locally open morphism of semi-graphs of anabelioids, 2.2 , ii

locally trivial morphism of semi-graphs of anabelioids, 2.2 , ii

log curve (stable, smooth), §0, Curves

maximal subgraph, $\S 1$

minimal-adjoint, $\S 0$, Categories

mobile, $\S 0$, Categories

moduli stack, §0, Curves

morphism of arithmetic semi-graphs of anabelioids (properties of), 5.1, iv

morphism of QD-pairs, A.3, ii

morphism of quasi-temperoids, A.1, iii

morphism of (semi-)graphs of anabelioids, 2.1

morphism of semi-graphs, $\S 1$

morphism of temperoids, 3.1 , iii

NL-morphism, 4.2, i

nondegenerate object, A.1, ii

normalizer, §0, Topological Groups

nuclear object, 4.2 , i 
open edge, $\S 1$

open object, after 4.1 .2

$\pi_{1}$-epimorphic approximator, 2.3 , ii

$\pi_{1}$-monomorphism, 2.1

piecewise faithfully, 4.1, i

proper morphism of semi-graphs, $\S 1$

properties of an arithmetic semi-graph of anabelioids, 5.1, iii pro- $\Sigma$-completion, 2.9 , ii

pro-temperoids, 3.4 .2

QD-pair, A.3, i

quasi-anabelioid, A.4.2

quasi-coherent, 2.3 , iii

quasi-connected, $\S 0$, Categories

quasi-geometric, 3.8

quasi-morphism of quasi-temperoids, A.1, iii

quasi-temperoid (connected), A.1, i, ii

quotient of a QD-pair, A.3, iii

rigid, $§ 0$, Categories

semi-graph, $\S 1$

(semi-)graph of anabelioids, 2.1

$\Sigma$-integer, 2.9, i

slim, $\S 0$, Categories

strongly connected, A.3, i

structure morphism, 4.1, iii

sub-coverticial (totally, universally), 2.4, iii

subjoint, $\S 1$

sub-semi-graph, $\S 1$

temp-, after 6.9.1

tempered covering of a semi-graph of anabelioids, 3.5, ii

tempered DLoc-type, 6.7

tempered fundamental group of a temperoid, 3.2.1

tempered object, after 4.1.2

tempered topological group, 3.1, i 
temperification, 3.1.4

temperoid, 3.1, ii

temp-slim (relatively), 3.4, ii

topological space associated to a semi-graph, $\S 1$

totally epimorphic, §0, Categories

tree, $\S 1$

universal graph-covering, $\S 1$

universal pro-finite étale covering, 2.2.1

untangled semi-graph, $\S 1$

vertex, $\S 1$

verticial cardinality, $\S 1$

verticial degree, 4.2 , i

verticial length, 4.2 , i

verticially iso-excisive, 4.1 , iv

verticially slim, 2.4 , ii

verticial morphism, 4.2 , i

verticial portion, $\S 1$

verticial restriction, $\S 1$

verticial subgroup, 3.7 , i

verticial subgroup (arithmetic case), 5.3, iii

weakly connected, A.3, i

\section{References}

[André] André, Y., On a Geometric Description of $\operatorname{Gal}\left(\overline{\mathbb{Q}}_{p} / \mathbb{Q}_{p}\right)$ and a $p$-adic Avatar of $\widehat{G} T$, Duke Math. J., 119 (2003), 1-39.

[DM] Deligne, P. and Mumford, D., The irreducibility of the moduli space of curves of given genus, IHES Publ. Math., 36 (1969), 75-109.

[Dub] Dubuc, E., On the representation theory of Galois and atomic topoi, J. Pure Appl. Algebra, 186 (2004), 233-275.

[HR] Herfort, W. and Ribes, L., Torsion elements and centralizers in free products of profinite groups, J. Reine Angew. Math., 358 (1985), 155-161.

[Knud] Knudsen, F. F., The projectivity of the moduli space of stable curves, II, Math. Scand., 52 (1983), 161-199.

[Milne] Milne, J. S., Étale Cohomology, Princeton Mathematical Series, 33, Princeton University Press, 1980.

[Mzk1] Mochizuki, S., The geometry of the compactification of the Hurwitz scheme, Publ. RIMS, Kyoto Univ., 31 (1995), 355-441. 
[Mzk2] Mochizuki, S., The local pro-p anabelian geometry of curves, Invent. Math., 138 (1999), 319-423.

[Mzk3] The absolute anabelian geometry of hyperbolic curves, Galois Theory and Modular Forms, Kluwer Academic Publishers (2003), pp. 77-122.

[Mzk4] , The geometry of anabelioids, Publ. RIMS, Kyoto Univ., 40 (2004), $819-881$

[Mzk5] Topics surrounding the anabelian geometry of hyperbolic curves, Galois Groups and Fundamental Groups, Mathematical Sciences Research Institute Publications 41, Cambridge University Press (2003), pp. 119-165.

[Mzk6] Categorical representation of locally noetherian log schemes, Adv. Math. 188 (2004), 222-246.

[Mzk7] Categories of log schemes with archimedean structures, J. Math. Kyoto Univ., 44 (2004), 891-909.

[Mzk8] $\longrightarrow$ Galois sections in absolute anabelian geometry, Nagoya Math. J., 179 (2005), 17-45.

[Naka] Nakamura, H., Galois rigidity of algebraic mappings into some hyperbolic varieties, Internat. J. Math., 4 (1993), 421-438.

[NTs] Nakamura, H. and Tsunogai, H., Some finiteness theorems on galois centralizers in pro-l mapping class groups, J. Reine Angew. Math., 441 (1993), 115-144.

[RZ] Ribes and Zaleskii, Profinite Groups, Ergeb. Math. Grenzgeb., 3, Springer-Verlag, 2000.

[Serre] Serre, J.-P., Trees, Springer Monogr. Math., Springer-Verlag, 2003.

[SGA1] Revêtement étales et groupe fondamental, Séminaire de Géometrie Algébrique du Bois Marie 1960-1961 (SGA1), dirigé par A. Grothendieck, augmenté de deux exposés de M. Raynaud, Lecture Notes in Math., 224, Springer-Verlag, 1971.

[Tama1] Tamagawa, A., On tame fundamental groups of curves over algebraically closed fields of characteristic > 0, Galois Groups and Fundamental Groups, Mathematical Sciences Research Institute Publications 41, Cambridge University Press (2003), pp. 47-105.

[Tama2] Tamagawa, A., Resolution of nonsingularities of families of curves, Publ. RIMS, Kyoto Univ., 40 (2004), 1291-1336. 